\title{
Radio Continuum Surveys with Square Kilometre Array Pathfinders
}

Ray P. Norris ${ }^{1,2,36}$, J. Afonso ${ }^{3}$, D. Bacon ${ }^{4}$, Rainer Beck ${ }^{5}$, Martin Bell ${ }^{2,6,7}$, R. J. Beswick ${ }^{8}$, Philip Best ${ }^{9}$,

Sanjay Bhatnagar ${ }^{10}$, Annalisa Bonafede ${ }^{11}$, Gianfranco Brunetti ${ }^{12}$, Tamás Budavári ${ }^{13}$, Rossella Cassano ${ }^{12}$, J. J. Condon ${ }^{10}$, Catherine Cress ${ }^{14}$, Arwa Dabbech ${ }^{15}$, I. Feain ${ }^{1,7}$, Rob Fender ${ }^{6}$, Chiara Ferrari ${ }^{15}$, B. M. Gaensler ${ }^{2,7}$, G. Giovannini ${ }^{12}$, Marijke Haverkorn ${ }^{16,17}$, George Heald ${ }^{18}$, Kurt Van der Heyden ${ }^{19}$, A. M. Hopkins ${ }^{20}$, M. Jarvis ${ }^{14,21,22}$, Melanie Johnston-Hollitt ${ }^{23}$, Roland Kothes ${ }^{24}$, Huib Van Langevelde ${ }^{16,25}$, Joseph Lazio $^{26}$, Minnie Y. Mao ${ }^{1,20,27,28}$, Alejo Martínez-Sansigre ${ }^{4}$, David Mary ${ }^{15}$, Kim Mcalpine ${ }^{21,29}$, E. Middelberg ${ }^{30}$, Eric Murphy ${ }^{31}$, P. Padovani ${ }^{32}$, Zsolt Paragi ${ }^{25}$, I. Prandoni ${ }^{12}$, A. Raccanelli ${ }^{4,26,33}$, Emma Rigby $^{34}$, I. G. Roseboom ${ }^{9}$, H. Röttgering ${ }^{16}$, Jose Sabater ${ }^{9}$, Mara Salvato ${ }^{11}$, Anna M. M. Scaife ${ }^{6}$, Richard Schilizzi $^{8}$, N. Seymour ${ }^{1}$, Dan J. B. Smith ${ }^{21}$, Grazia Umana ${ }^{35}$, G.-B. Zhao ${ }^{4}$ and Peter-Christian Zinn ${ }^{1,30}$

${ }^{1}$ CSIRO Astronomy \& Space Science, PO Box 76, Epping, NSW 1710, Australia

${ }^{2}$ ARC Centre of Excellence for All-sky Astrophysics (CAASTRO), Redfern, NSW 2016, Australia

${ }^{3}$ Centro de Astronomia e Astrofísica da Universidade de Lisboa, Observatório Astronómico de Lisboa, Tapada da Ajuda, 1349-018 Lisboa, Portugal

${ }^{4}$ Institute of Cosmology and Gravitation, University of Portsmouth, Dennis Sciama Building, Burnaby Road, Portsmouth PO1 3FX, UK

${ }^{5}$ Max Planck Institut fur Radioastronomie, Auf dem Hugel 69, Bonn, Germany

${ }^{6}$ School of Physics \& Astronomy, University of Southampton, Southampton SO17 1BJ, UK

${ }^{7}$ Sydney Institute for Astronomy, School of Physics, The University of Sydney, NSW 2006, Australia

${ }^{8}$ Jodrell Bank Centre for Astrophysics, School of Physics and Astronomy, The University of Manchester, Manchester M13 9PL, UK

${ }^{9}$ Institute for Astronomy, University of Edinburgh, Royal Observatory, Blackford Hill, Edinburgh EH9 3HJ, UK

${ }^{10}$ National Radio Astronomy Observatory, 520 Edgemont Road, Charlottesville, VA 22903, USA

${ }_{11}^{11}$ Max Planck Institute for Plasma Physics, Boltzmannstr. 2 D-85748 Garching, Germany

${ }^{12}$ INAF-IRA, Via P. Gobetti 101, 40129 Bologna, Italy

${ }^{13}$ Department of Physics and Astronomy, The Johns Hopkins University, 3400 North Charles Street, Baltimore, MD 21218, USA

${ }^{14}$ Physics Department, University of the Western Cape, Cape Town 7535, South Africa

${ }^{15}$ Laboratoire Lagrange, UMR 7293, Université de Nice Sophia-Antipolis, CNRS, Observatoire de la Côte d'Azur, 06300, Nice, France

${ }^{16}$ Leiden Observatory, Leiden University, PO Box 9513, 2300 RA Leiden, The Netherlands

${ }^{17}$ Department of Astrophysics/IMAPP, Radboud University Nijmegen, PO Box 9010, 6500 GL Nijmegen, The Netherlands

${ }^{18}$ ASTRON, Postbus 2, 7990 AA Dwingeloo, The Netherlands

${ }^{19}$ Astrophysics, Cosmology \& Gravity Centre, Department of Astronomy, University of Cape Town, Private Bag X3, Rondebosch 7701, South Africa

${ }^{20}$ Australian Astronomical Observatory, PO Box 296, Epping, NSW 1710, Australia

${ }^{21}$ Centre for Astrophysics Research, Science \& Technology Research Institute, University of Hertfordshire, Hatfield, Herts, UK

${ }^{22}$ Oxford Astrophysics, Denys Wilkinson Building, University of Oxford, Keble Rd, Oxford OX1 3RH, UK

${ }^{23}$ School of Chemical \& Physical Sciences, Victoria University of Wellington, PO Box 600, Wellington 6140, New Zealand

${ }^{24}$ National Research Council of Canada, National Science Infrastructure, Dominion Radio Astrophysical Observatory, PO Box 248, Penticton, BC V2A

$6 \mathrm{~J} 9$, Canada

${ }^{25}$ Joint Institute for VLBI in Europe, Postbus 2, 7990 AA DWINGELOO, The Netherlands

${ }^{26}$ Jet Propulsion Laboratory, California Institute of Technology, Pasadena, CA 91109, USA

${ }^{27}$ National Radio Astronomy Observatory, PO Box 0, Socorro, NM 87801, USA

${ }^{28}$ School of Mathematics \& Physics, University of Tasmania, Private Bag 37, Hobart 7001, Australia

${ }^{29}$ Department of Physics and Electronics, Rhodes University, Grahamstown 6140, South Africa

${ }^{30}$ Astronomisches Institut, Ruhr-Universität Bochum, Universitätsstr. 150, 44801 Bochum, Germany

${ }^{31}$ Carnegie Observatories, 813, Santa Barbara St., Pasadena, CA 91101, USA

${ }^{32}$ European Southern Observatory, Karl-Schwarzschild-Str. 2, D-85748 Garching bei München, Germany

${ }^{33}$ California Institute of Technology, Pasadena, CA 91125, USA

${ }^{34}$ School of Physics \& Astronomy, University of Nottingham, Nottingham NG7 2RD, UK

${ }^{35}$ INAF-Catania Astrophysical Observatory, Via S. Sofia 78, 95123 Catania, Italy

${ }^{36}$ Corresponding author. Email: Ray.Norris@csiro.au

(Received June 17, 2012; Accepted October 22, 2012; Online Publication March 27, 2013)

\begin{abstract}
In the lead-up to the Square Kilometre Array (SKA) project, several next-generation radio telescopes and upgrades are already being built around the world. These include APERTIF (The Netherlands), ASKAP (Australia), $e$-MERLIN
\end{abstract}


(UK), VLA (USA), e-EVN (based in Europe), LOFAR (The Netherlands), MeerKAT (South Africa), and the Murchison Widefield Array. Each of these new instruments has different strengths, and coordination of surveys between them can help maximise the science from each of them. A radio continuum survey is being planned on each of them with the primary science objective of understanding the formation and evolution of galaxies over cosmic time, and the cosmological parameters and large-scale structures which drive it. In pursuit of this objective, the different teams are developing a variety of new techniques, and refining existing ones. To achieve these exciting scientific goals, many technical challenges must be addressed by the survey instruments. Given the limited resources of the global radio-astronomical community, it is essential that we pool our skills and knowledge. We do not have sufficient resources to enjoy the luxury of re-inventing wheels. We face significant challenges in calibration, imaging, source extraction and measurement, classification and crossidentification, redshift determination, stacking, and data-intensive research. As these instruments extend the observational parameters, we will face further unexpected challenges in calibration, imaging, and interpretation. If we are to realise the full scientific potential of these expensive instruments, it is essential that we devote enough resources and careful study to understanding the instrumental effects and how they will affect the data. We have established an SKA Radio Continuum Survey working group, whose prime role is to maximise science from these instruments by ensuring we share resources and expertise across the projects. Here we describe these projects, their science goals, and the technical challenges which are being addressed to maximise the science return.

Keywords: radiotelescopes - surveys - galaxy evolution - cosmology

\section{INTRODUCTION AND BACKGROUND}

The Square Kilometre Array (SKA) is a proposed major internationally funded radio telescope (Dewdney et al. 2009) which is expected to be completed in the next decade. It will be many times more sensitive than any existing radio telescope, covering centimetre to metre wavelengths, and will answer fundamental questions about the Universe by surveying the radio sky and studying individual objects in detail (Carilli \& Rawlings 2004). It will consist of many antennas, constituting an effective collecting area of about one square kilometre, deployed over two sites in Australia and South Africa.

In the lead-up to the SKA, several next-generation radio telescopes and upgrades are being constructed around the world, including APERTIF (The Netherlands), ASKAP (Australia), $e$-MERLIN (UK), e-EVN (based in Europe), the Low Frequency Radio Array (LOFAR, The Netherlands), Meerkat (South Africa), the Murchison Widefield Array (MWA; Australia), and the Karl G. Jansky Very Large Array (VLA, USA). Large continuum surveys are being planned for many of these telescopes.

The predicted sensitivities and areas for these surveys are shown in Figure 1, alongside existing 1.4-GHz continuum radio surveys. The largest published radio survey, shown in the top right, is the wide but shallow NRAO VLA Sky Survey (NVSS; Condon et al. 1998). The most sensitive published radio survey is the deep but narrow Lockman Hole observation (Owen \& Morison 2008) in the lower left, which has recently been overtaken by an even deeper observation of the same field (Condon et al. 2012). All current surveys are bounded by a diagonal line that roughly marks the limit of available telescope time of current-generation radio telescopes. The region to the left of this line is currently unexplored, and this area of observational phase space presumably contains as many potential new discoveries as the region to the right.
Most of these projects have multiple science goals, but they have one goal in common, which is to survey the radio continuum emission from galaxies, in order to understand the formation and evolution of galaxies over cosmic time, and the cosmological parameters and large-scale structures that drive it. In pursuit of this goal, the different teams are developing techniques such as multi-scale deconvolution, source extraction and classification, and multiwavelength cross-identification. Furthermore, these projects share specific scientific goals, some of which require further definition before a well-planned survey can be executed. Finally, each of these new instruments has different strengths, and coordination of surveys between them can help maximise the scientific return from each.

The radio continuum surveys planned for these instruments typically reach orders of magnitude deeper than traditional surveys. Consequently, the resulting surveys will be far more than just a deep version of existing surveys, but will be qualitatively different, in most cases being dominated by star-forming (SF) galaxies. For example, only a total of about $10 \mathrm{deg}^{2}$ of the sky has been surveyed at $1.4 \mathrm{GHz}$ to the planned $10 \mu \mathrm{Jy}^{\text {beam }}{ }^{-1}$ rms of many of these surveys, in fields such as the Hubble, Chandra, ATLAS, COSMOS, and Phoenix deep fields (Hopkins et al. 2003; Huynh et al. 2005; Biggs \& Ivison 2006; Norris et al. 2006; Middelberg et al. 2008b; Miller et al. 2008; Schinnerer et al. 2007; Morrison et al. 2010; J. K. Banfield et al., in preparation; G. Guglielmino et al., in preparation; C. A. Hales et al., in preparation).

Surveys at this depth extend beyond the traditional domains of radio astronomy, where sources are predominantly radio-loud galaxies and quasars, into the regime of SF galaxies. At this depth, even the most common active galactic nuclei (AGNs) are radio-quiet AGNs, which make up most of the X-ray extragalactic sources. As a result, the role of radio astronomy is changing. Previous radio-astronomical 


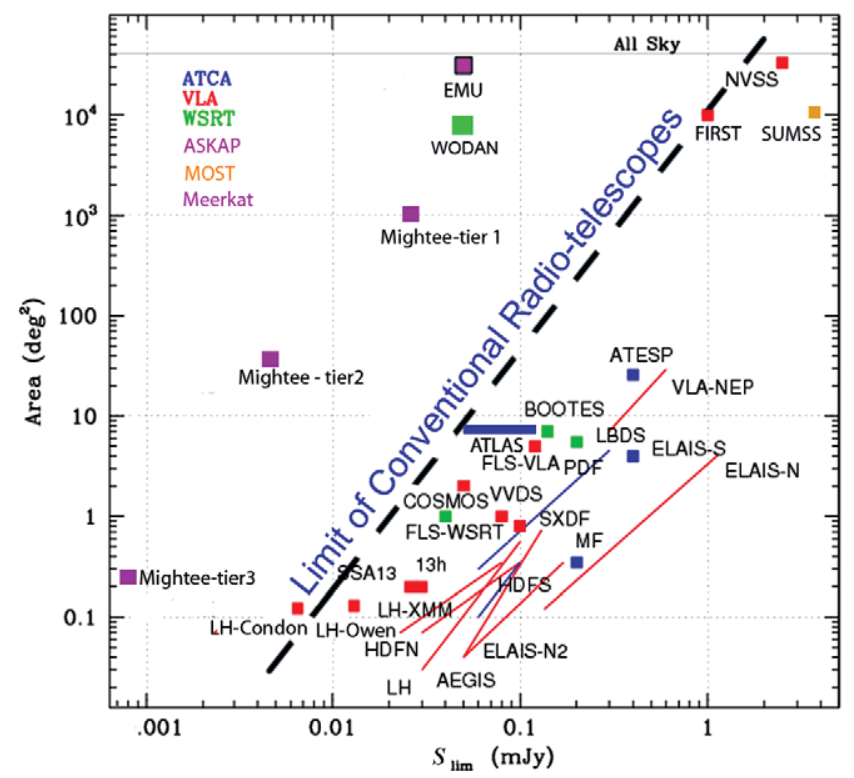

Figure 1. Comparison of existing and planned deep 1.4-GHz radio surveys. The horizontal axis shows the $5 \sigma$ sensitivity and the vertical axis shows the sky coverage. The diagonal dashed line shows the approximate envelope of existing surveys, which is largely determined by the availability of telescope time. For example, to extend NVSS to the sensitivity of EMU would have required over 600 years of (pre-upgrade) VLA time, so in practice this would not have been possible, and this line therefore represents a hard limit to the sensitivity of traditional surveys. The squares in the top left represent the new radio surveys discussed in this paper. Surveys at other frequencies are not shown in this diagram, as their relative sensitivity depends on the assumed spectral index of the sources, although we do include SUMMS at $843 \mathrm{MHz}$, without making any correction for spectral index. A similar comparison of low-frequency surveys can be found in Tingay et al. (2012).

surveys had their greatest impact in the niche area of radio-loud AGNs. For example, the numbers of sources in NVSS (Condon et al. 1998) and FIRST (Becker, White, \& Helfand 1995) are overwhelmingly dominated by AGNs, and only a small fraction of their sources are SF galaxies. In contrast, most sources detected by the next-generation surveys are SF galaxies, so that these surveys are dominated by the same galaxies as are studied by optical and infrared (IR) surveys. As a result, these next-generation radio surveys are an increasingly important component of multiwavelength studies of galactic evolution.

These surveys, however, will also encounter a new set of technical and scientific challenges. For example, the new continuum surveys such as the Evolutionary Map of the Universe (EMU; see Section 2.3) and the Westerbork Observations of the Deep APERTIF Northern-Sky (WODAN; see Section 2.1) should cover the whole sky at sensitivities previously attained only in small fields $\left(\sim 10 \mu \mathrm{Jy} \mathrm{beam}^{-1} \mathrm{rms}\right)$ by instantaneously covering wide fields $\left(\sim 30 \mathrm{deg}^{2}\right)$ with large bandwidths $(\sim 300 \mathrm{MHz})$ and long integration times $(\sim 12$ h), and not by virtue of larger collecting areas or lower system noise temperatures. To reach the sensitivities predicted by the radiometer equation, these surveys must have exceptionally high dynamic ranges (e.g., about $42 \mathrm{db}$ for EMU) and low systematic errors. This requires very high quality hardware, calibration techniques, and imaging algorithms. In particular, the primary beams must have $<1 \%$ uncertainties in reconstructed position, size, and shape. The ASKAP dishes, for example, appear to have good surface and pointing accuracies, and their three-axis mounts maintain a constant footprint of the primary beam on the sky. The electronically formed primary beams of phased-array feeds also have to be very stable, and the survey astronomers need to work closely with the engineers to ensure that amplitude and phase calibration on strong sources in the array field of view (FOV) can be transferred from one primary beam to another. Finally, the success of a survey in the astronomical community depends as much on the survey results being made available to everyone in an easily-accessible fashion (such as through a web portal, or virtual observatory tools, and with useful and intuitive query engines) as on whether the survey actually meets every performance goal, such as sensitivity.

Moreover, some of these projects differ from earlier surveys in that one goal is to cross-identify the detected radio sources with major surveys at other wavelengths, and produce public-domain Virtual Observatory (VO) accessible catalogues as 'value-added' data products. This is facilitated by the growth in the number of major surveys spanning all wavelengths, discussed below in Section 4.6.

To address these challenges, a new group, the SKA PAthfinder Radio Continuum Survey (SPARCS) Working Group has been established, with the following goals:

- To coordinate developments of techniques, to avoid duplication of effort and ensure that each project has access to best practice.

- To hold cross-project discussions of the specific scientific goals, to ensure cross-fertilisation of ideas and optimum survey strategies.

- To coordinate the surveys in their choice of area, depth, location on the sky, and other survey parameters, to maximise the scientific return from the surveys.

- To distil the SKA pathfinder experiences in order to provide the most relevant and up to date input for SKA planning.

SPARCS was initially proposed during the SKA meeting in Manchester in 2010 March, was formed in 2010 May, and held its first meeting at the Lorentz Center in Leiden, The Netherlands, in 2011 February. This review paper documents the outcomes of that workshop, together with related developments since the workshop. The authors are the speakers and facilitators of the workshop, together with other domain experts who were unable to attend the workshop in person.

This paper describes the SKA pathfinder continuum surveys in Section 2, setting out their scientific goals in Section 3, and identifying the challenges to achieve these goals in Section 4. Section 5 concludes by summarising how these challenges are being addressed, and how SPARCS is helping to coordinate and facilitate this process. We refer to the 
collection of all surveys discussed in this paper as the SPARCS surveys, and the sources detected in those surveys as the SPARCS sources. We assume the convention for spectral index $\alpha$ that $S \propto \nu^{\alpha}$ throughout.

\section{PATHFINDERS AND THEIR CONTINUUM SURVEYS}

\subsection{APERTIF-WODAN}

APERTIF, the new Phased Array Feed (PAF) receiver system for the Westerbork Synthesis Radio Telescope (WSRT), will dramatically enlarge the instantaneous FOV of the WSRT (Oosterloo et al. 2009) by replacing the current single frontend feeds by PAFs. Each of the PAFs consists of 121 Vivaldi elements and will detect the radiation field (in dual polarisation) in the focal plane of each dish over an area of about one square metre at an observing frequency of $1.4 \mathrm{GHz}$. Because of this, many beams can be formed simultaneously for each dish making it possible to image an area of about $8 \mathrm{deg}^{2}$ on the sky, which is an increase of about a factor of 30 compared to the current WSRT. Its large 300-MHz bandwidth will not only cater for sensitive continuum imaging, but is also crucial for efficient $\mathrm{H}$ I and $\mathrm{OH}$ emission surveys and for studies of polarised emission from large areas.

The WODAN survey (Röttgering et al. 2010b) will use APERTIF to survey the northern $25 \%$ of the sky (i.e. north of declination $+30^{\circ}$ ) that is inaccessible to ASKAP, to a target rms sensitivity of $10 \mu \mathrm{Jy}$ beam $^{-1}$ at a spatial resolution of 15 arcsec, although confusion noise with a 15- arcsec beam may increase the observed rms noise level to about $20 \mu \mathrm{Jy}_{\text {beam }^{-1}}$.

\subsection{APERTIF-BEOWULF and APERTIF- FRIGG}

BEOWULF and FRIGG are both polarisation projects proposed for APERTIF.

The BEOWULF (B-field Estimation and Observational Wide-field Understanding of Large-scale Faraday-structure) survey (Scaife et al. 2010) will use APERTIF to survey an area of $64 \mathrm{deg}^{2}$ within the Perseus-Pisces supercluster of galaxies in order to construct a finely spaced rotation measure (RM) grid tuned for cosmological and large-scale structure studies of magnetism within both clusters and the filamentary inter-cluster medium. This survey will complement other wide area polarisation surveys: by measuring RMs to 5000 background sources it will give an RM-grid sampling of $3.5 \mathrm{arcmin}$. The BEOWULF survey will provide vital insights into whether magnetic fields were primordial in clusters of galaxies, or whether the galaxies themselves later injected the field. The survey is ideally suited to APERTIF whose wide FOV enables observations of large-scale structures, and whose frequency coverage is well matched to the comparatively high RMs expected from such a region.

The FRIGG (Faraday Rotation Investigation of Galaxies and Groups) survey (Beck et al. 2010) will observe large areas around four large northern nearby spiral galaxies, the bridge between M 31 and M 33, and five galaxy groups. Its goal is to measure grids of Faraday RMs of background sources and faint diffuse emission, in order to unravel the extent and dynamical importance of cosmic magnetic fields in galaxies and in intergalactic space in galaxy groups.

\subsection{ASKAP-EMU}

The Australian SKA Pathfinder (ASKAP; Johnston et al. 2007, 2008; Deboer et al. 2009) is a new radio telescope being built on the Australian SKA site in Western Australia, at the Murchison Radio-astronomy Observatory, with a planned completion date of 2013-14. It will consist of 36 12-metre antennas distributed over a region $6 \mathrm{~km}$ in diameter. Each antenna is equipped with a PAF (Bunton \& Hay 2010) of 96 dual-polarisation pixels operating in a frequency band of 700-1 $800 \mathrm{MHz}$. As a result, ASKAP will have an FOV up to $30 \mathrm{deg}^{2}$. To ensure good calibration, the antennas are a novel three-axis design, with the feed and reflector rotating to mimic the effect of an equatorial mount, ensuring a constant position angle of the PAF and sidelobes on the sky.

In continuum mode, ASKAP will observe a $300-\mathrm{MHz}$ band, split into 1-MHz channels, with full Stokes parameters measured in each channel. The data will be processed in a multi-frequency synthesis mode, in which data from each channel are correctly gridded in the $u v$ plane. As well as producing images and source catalogues, the processing pipeline will also measure spectral index, spectral curvature, and all polarisation products across the band. All data processing steps, from the output of the correlator to science-quality images, spectra, and catalogues, are performed in automated pipelines (Cornwell et al. 2011) running on a highly distributed parallel processing computer. These steps include flagging bad data, calibration, imaging, source-finding, and archiving.

EMU (Norris et al. 2011b) will use ASKAP to make a deep (10 $\mu \mathrm{Jy}^{\text {beam }}{ }^{-1} \mathrm{rms}$ ) radio continuum survey of the entire southern sky, extending as far north as $+30^{\circ}$. EMU will cover roughly the same fraction $(75 \%)$ of the sky as the benchmark NVSS survey (Condon et al. 1998), but will be 45 times more sensitive, and will have an angular resolution (10 arcsec) 4.5 times better. Because of the excellent shortspacing $u v$ coverage of ASKAP, EMU will also have higher sensitivity to extended structures. Like most radio surveys, EMU will adopt a $5 \sigma$ cut-off, leading to a source detection threshold of $50 \mu \mathrm{Jy}$ beam $^{-1}$. EMU is expected to generate a catalogue of about 70 million galaxies, and all radio data from the EMU survey will be placed in the public domain as soon as the data quality has been assured.

Together, EMU and WODAN (see Section 2.1) will provide full-sky 1.3-GHz imaging at $\sim 10-15$ arcsec resolution to an rms noise level of $10 \mu \mathrm{Jy}_{\text {beam }}{ }^{-1}$, providing an unprecedented sensitive all-sky radio survey as a legacy for astronomers at all wavelengths. The EMU and WODAN surveys will overlap by a few degrees of declination to provide 
a comparison and cross-validation, to ensure consistent calibration, and to check on completeness and potential sources of bias between the surveys.

\subsection{ASKAP-POSSUM}

POSSUM (POlarisation Sky Survey of the Universe's Magnetism) is an all-sky ASKAP survey of linear polarisation (Gaensler et al. 2010). It is expected that POSSUM will be commensal with EMU, and that the two surveys will overlap considerably in their analysis pipelines and source catalogues. POSSUM will provide a catalogue of polarised fluxes and Faraday RMs for approximately three million compact extragalactic sources. These data will be used to determine the large-scale magnetic field geometry of the Milky Way, to study the turbulent properties of the interstellar medium (ISM), and to constrain the evolution of intergalactic magnetic fields as a function of cosmic time. POSSUM will also be a valuable counterpart to EMU, in that it will provide polarisation properties or upper limits to polarisation for all sources detected by EMU.

\section{5 $e$-MERLIN}

The $e$-MERLIN array (Garrington et al. 2004), operated by the University of Manchester, is a significant upgrade to the existing telescopes which form the MERLIN array in the UK. Consisting of seven telescopes, spread across the UK with a maximum baseline of $217 \mathrm{~km}, e$-MERLIN provides high angular resolution (10-150 mas) imaging and spectroscopy in three broad cm-wavebands $(1.3-1.8,4-8$, and $22-24 \mathrm{GHz})$. The $e$-MERLIN project includes a major upgrade to the existing telescope hardware, including the installation of new receivers, analogue and digital electronics, optical-fibre links to each telescope and a powerful new digital correlator at Jodrell Bank Observatory. This upgrade increases the usable bandwidth by more than two orders of magnitude, compared with the old MERLIN system, so that the continuum sensitivity is increased by a factor of between 10 and 30. In addition, the increase in bandwidth will enable multi-frequency synthesis, dramatically improving the $u v$ coverage for continuum observations and enabling simultaneous spectral-index imaging.

The $e$-MERLIN upgrade is well underway with new receivers in service, the dedicated optical fibre network and the digital transmission equipment operational and returning $30 \mathrm{Gbit} \mathrm{s}^{-1}$ of data from each telescope back to a new correlator. Currently, $e$-MERLIN is undergoing the final stages of instrument commissioning and will be fully operational during 2013.

These upgrades to the system allow $e$-MERLIN to typically provide $\mu \mathrm{Jy}$ sensitivity with up to $4 \mathrm{GHz}$ of instantaneous bandwidth. This sensitive continuum imaging can be simultaneously combined with powerful spectral line and polarisation capabilities provided by the new highly flexible
WIDAR correlator which is a twin of the new VLA correlator and has been developed by DRAO in Canada.

The $e$-MERLIN array is an important SKA pathfinder instrument covering a unique scientific niche due to its baseline lengths, which are intermediate between those provided by the VLA and VLBI arrays. Technically this provides a testbed for the development of fibre-based data transport and time distribution over distances spanning many hundreds of kilometres. Scientifically, results stemming from its high angular resolution will provide significant input into the eventual locations of dish receptors as part of SKA Phase 1.

$e$-MERLIN is an open-user instrument with twice yearly proposal deadlines. As with many other new radio facilities, however, it has pre-allocated a large fraction of observing time to large key-science projects. Eleven large $e$-MERLIN 'legacy' projects ${ }^{1}$ have been allocated a total of $5000 \mathrm{~h}$ (approximately $50 \%$ of the available observing time) over the first five semesters of operation. These key projects span a wide range of astrophysics from studies of planet formation, pulsar proper motions, stars, nearby normal and SF galaxies, classical radio galaxies, strong gravitational lenses, galaxy clusters, and the physics of high-redshift star formation and AGN-dominated galaxies. In each of these programs, the strengths of $e$-MERLIN, in terms of its sensitivity and critically high angular resolution at centimetre wavelengths, are being exploited to provide a spatially resolved view of these sources, complementing studies planned or underway with other SKA pathfinder instruments, and new facilities at other wavelengths.

\subsection{VLA}

The Karl G. Jansky Very Large Array (VLA: Napier 2006) represents a major upgrade to the VLA. Although the upgraded VLA was not designed to be a survey telescope or an SKA pathfinder, its high sensitivity, continuous frequency coverage, flexible wideband correlator, and scaled array configurations make the VLA a useful complement to the dedicated pathfinders, and to the all-sky continuum surveys that are being planned. For example, the VLA could observe 'reference fields' covering a few small areas of sky with higher angular resolution (e.g. 5 arcsec) and sensitivity (e.g. $5 \mu \mathrm{Jy}$ beam $^{-1} \mathrm{rms}$ ) than the 1.4-GHz EMU and WODAN surveys in order to join those surveys seamlessly and provide a reference for resolution corrections, flux densities, positions, etc. of the faintest survey sources.

The VLA is a general-purpose user instrument, open to researchers around the world. The VLA upgrade is approaching completion, and algorithms for making 20-cm images with large fractional bandwidth and high dynamic range are now being tested on real data. The ultimate continuum sensitivity limits (source confusion or dynamic range) of the full SKA depend on the surface density on the sky of sub- $\mu$ Jy sources. If dynamic range permits, by imaging a single field at $20 \mathrm{~cm}$

\footnotetext{
${ }^{1}$ http://www.e-merlin.ac.uk/legacy/
} 
to the confusion limit with 5- arcsec resolution, the VLA in its B configuration will place a tight statistical constraint on the source density of objects fainter than $1 \mu \mathrm{Jy}$.

\section{7 e-VLBI}

The SKA will be a real-time instrument with preferably $25 \%$ of its collecting area forming long baselines that extend up to $3000 \mathrm{~km}$. Data caching and transportation, distribution of the clock signal and operations of this array will present a great technical challenge. The European VLBI Network (EVN) as an SKA pathfinder is addressing these issues within the framework of the NEXPReS project (http://www.nexpres.eu/). The primary goal of NEXPReS is to support and develop real-time electronic-VLBI (e-VLBI) operations in the EVN and on global scales.

Science observations with the e-EVN have routinely been carried out since 2006 . The possibility of operating a realtime VLBI array with baselines exceeding $12000 \mathrm{~km}$ was recently demonstrated by Giroletti et al. (2011). Relaxing the data storage limitations at the telescopes creates an obvious advantage for the EVN. This allows for more flexible operations to carry out transient science (see Section 3.7), it may provide seamless data transport at $>1$ Gbps data rates for superior sensitivity, and makes it possible to conduct automated observations for efficient VLBI surveys or to respond to external triggers.

The importance of long baselines (and even the possibility of baselines to space) for the SKA has been discussed by Fomalont \& Reid (2004) and Gurvits (2004). Present-day observations of faint, compact radio sources are beginning to shed light on the science that could be done with one or two orders of magnitude better sensitivity. The mJy sky is thought to be dominated by AGNs, compact on milliarcsec scales, while in the sub-mJy regime SF galaxies become increasingly important, and a number of objects are now known which show both strong AGN activity and vigorous star formation activity (e.g. Afonso et al. 2001; Pérez-Torres et al. 2010; Norris et al. 2012). VLBI, and the long baselines of SKA, will play a key role in distinguishing between AGN and SF galaxies.

The long baselines of the SKA will be particularly important for studying the details of feedback processes between AGN and star formation (Croton et al. 2006), the role of binary AGN mergers in galaxy evolution and feedback (e.g. Dotti, Sesana \& Decarli 2012), the first supermassive black holes (SMBHs) at the highest redshifts (Falcke, Körding, \& Nagar 2004; Frey et al. 2010, 2011), and several other applications (see Godfrey et al. 2012 for a review).

In traditional VLBI observations, the FOV was severely limited by time- and bandwidth smearing. Since the sub-mJy sky is densely populated with radio sources, VLBI observations are moving from the traditional mode of observing single, bright, widely-separated objects to a mode which allows observations of wide fields. This transition poses a significant technological challenge, because the combina- tion of high resolution and wide fields requires high spectral and temporal resolution in correlation, to prevent loss of coherence arising from averaging effects (bandwidth and time smearing). Early wide-field VLBI experiments (e.g. Garrett, Wrobel \& Morganti 2005) were limited by the hardware VLBI correlators and by limited computing resources. Recently, software correlators running on cheap commodity computers have provided the power and flexibility needed for VLBI observations of wide fields (Deller et al. 2011).

Since sources detectable with VLBI, even in the sub-mJy regime, are relatively sparsely distributed, it has proved to be more efficient to generate multiple phase centres with small FOVs directed towards known locations of radio emission, rather than to image huge blank portions of the sky. Alternative, equivalent approaches have also been proved to be successful (Morgan et al. 2011). The multi-phase centre technique has only recently been brought to production (Middelberg et al. 2011b) but has already demonstrated that galaxies can contain a radioactive AGN even when an AGN is not evident in comprehensive radio, optical, and $\mathrm{X}$-ray observations (Middelberg et al. 2012).

Wide-field VLBI observations require new calibration steps to reach the full sensitivity of the data, such as primary beam corrections and multi-phase centre self-calibration, which help to make the technique useful for a wide range of applications. For example, the multi-phase centre technique can be used to image many sources around a faint target to aid in self-calibration. While no single source may be sufficiently strong for self-calibration, the ensemble of sources can provide a robust solution. The technique may also be used to provide an astrometric reference, or to image many background sources to probe a foreground object.

\subsection{LOFAR}

LOFAR, the Low Frequency Radio Array, is a pan-European radio phased-array telescope that is currently being commissioned. The two types of antennas, one optimised for 30-80 $\mathrm{MHz}$ and the other for $110-240 \mathrm{MHz}$, are grouped together in stations the size of soccer fields. Forty stations are distributed over an area of diameter of $100 \mathrm{~km}$ in the Netherlands, and a further eight stations are located in Germany, UK, Sweden, and France. The signals from the antennas are digitised to form many beams on the sky, making LOFAR an extremely efficient survey instrument. LOFAR has already generated images that are the deepest ever at these low frequencies.

A key motivation of LOFAR is to provide the entire international astronomical community with surveys of the radio sky that have a long-lasting legacy value for a broad range of astrophysical research. The LOFAR continuum survey (Röttgering et al. 2010a) will cover the northern half of the sky (i.e. north of declination $0^{\circ}$ ). LOFAR will be especially complementary to WODAN and EMU in surveying the sky at high sensitivity and resolution but at a much lower frequency.

The three fundamental areas of astrophysics that have driven the design of the planned LOFAR surveys are (i) the 


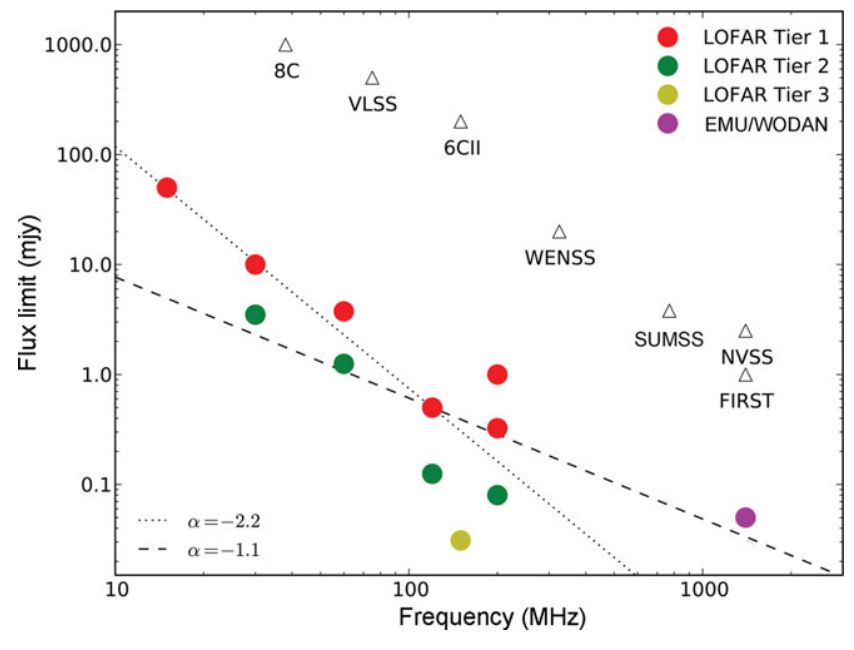

Figure 2. Flux limits $(5 \sigma)$ of the proposed LOFAR surveys compared with other radio surveys. The triangles represent existing surveys. The lines represent different power laws (with $\alpha=-1.6$ and -0.8 ) to illustrate how, depending on the spectral indices of the sources, the LOFAR surveys will compare with others.

formation of massive galaxies at the epoch of reionisation (EoR), (ii) magnetic fields and shocked hot gas associated with the first bound clusters of galaxies, and (iii) star formation processes in distant galaxies. The areas, depths, and frequencies of the surveys have been chosen to contain (i) $\sim 100$ powerful radio galaxies close to or at the EoR, (ii) $\sim 100$ radio haloes at the epoch when the first massive bound galaxy clusters appeared, and (iii) $\sim 100$ protoclusters. The resulting survey parameters are based on estimates of luminosity functions for powerful radio galaxies by Wilman et al. (2008), for radio haloes by Enßlin \& Röttgering (2002) and Cassano et al. (2010b), and for protoclusters by Venemans et al. (2007).

To achieve the goals of the LOFAR surveys, a three-tiered approach has been adopted (Röttgering et al. 2010a). Tier 1 is a $2 \pi$ steradian survey reaching an $\mathrm{rms}$ of $0.07 \mathrm{mJy}$ at 15-65 and 120-180 MHz, and is designed to detect $\sim 100$ cluster haloes at $z>0.6$ and $\sim 100 z>6$ radio galaxies. Tier 2 is a deep survey over $500 \mathrm{deg}^{2}$ at 30,60 , and $150 \mathrm{MHz}$. At $150 \mathrm{MHz}$, this will require 55 pointings, to be centred on the following science targets:

- 25 well-studied extragalactic fields with superb existing multiwavelength data;

- 15 fields centred on clusters or superclusters;

- 15 fields centred on nearby galaxies.

Tier 3 is an 'ultra-deep' survey at $150 \mathrm{MHz}$ covering a single pointing of $100 \mathrm{deg}^{2}$ reaching the confusion level of $7 \mu \mathrm{Jy}$ beam $^{-1} \mathrm{rms}$. Figure 2 shows the resulting depth versus frequency.

WODAN and EMU have an enormous synergy with the LOFAR surveys: virtually all the $5 \times 10^{7}$ radio sources from the LOFAR all-sky surveys will have their flux density at
$1400 \mathrm{MHz}$ measured (i.e. Figure 1). Together they will yield data for all radio loud AGNs in the northern hemisphere and most luminous starbursts up to $z=2$. The resulting densely populated radio colour-colour diagrams will be a powerful tool to spectrally discriminate between very rare radio sources with extreme radio spectra, such as diffuse emission from clusters and very distant radio galaxies. Nearby resolved sources will have spectral index and spectral curvature maps, a very rich source of information to constrain many physical parameters. As the combined surveys will cover the entire sky, measurements of the integrated SachsWolfe (ISW) effect, galaxy auto-correlation functions, and cosmic magnification will substantially tighten cosmological model parameters (Raccanelli et al. 2011).

LOFAR will also support a Key Science Project on Cosmic Magnetism $\left(\mathrm{MKSP}^{2}\right)$, which is likely to make significant contributions to our knowledge of the magnetic sky (see Section 3.5).

\subsection{MeerKAT/Mightee}

MeerKAT (Jonas 2009) is the South African SKA pathfinder telescope. MeerKAT Phase 1 will consist of 64 dishes, each $13.5 \mathrm{~m}$ in diameter, equipped with single-pixel receivers. An offset Gregorian dish configuration has been chosen because the unblocked aperture provides good optical performance, sensitivity, and imaging quality. It also facilitates the installation of multiple receiver systems in the primary and secondary focal areas. MeerKAT's 64-dish array layout will be distributed over two components. A dense inner component will contain $70 \%$ of the dishes with a Gaussian $u v$ distribution with a dispersion of $300 \mathrm{~m}$. The outer component will contain the remaining $30 \%$ of the dishes, having a Gaussian $u v$ distribution with a dispersion of $2500 \mathrm{~m}$ and a longest baseline of $8 \mathrm{~km}$. A potential future extension (MeerKAT Phase 2) could see seven additional dishes being added to extend the longest baselines to about $20 \mathrm{~km}$. MeerKAT will support a wide range of observing modes, including deep continuum, polarisation and spectral line imaging, pulsar timing, and transient searches. The plan is to provide standard data products, including an imaging pipeline. A number of 'data spigots' will also be available to support user-provided instrumentation.

Seventy per cent of observing time on MeerKAT, for the first few years, is allocated for large survey projects of $1000 \mathrm{~h}$ or more, while the remaining $30 \%$ is reserved for smaller PIdriven proposals (of which 5\% will be director's discretionary time). Proposals for key projects were solicited in 2010, and 10 projects were selected. Two (a pulsar timing and a deep $\mathrm{H}$ I survey) were chosen as top priority, and a further eight as second priority, one of which is MIGHTEE.

The MIGHTEE survey (van der Heyden \& Jarvis 2010) aims to probe to much fainter flux densities (0.1-

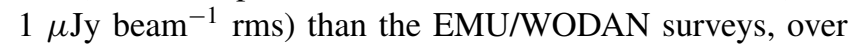
smaller areas $\left(\sim 35 \mathrm{deg}^{2}\right)$ at higher angular resolution, at an

\footnotetext{
${ }^{2}$ http://www.mpifr-bonn.mpg.de/staff/rbeck/MKSP/mksp.html
} 
observing frequency of $1.4 \mathrm{GHz}$. The higher sensitivity and resolution will enable exploration of AGN, SF galaxies, and galaxy clusters from the EoR through to the present day. The MIGHTEE survey strategy will follow a three-tiered approach both in survey area and in sensitivity, using the longer baselines to be able to probe below the confusion limit of the shorter baseline surveys such as EMU and WODAN.

Tier 1 will cover $1000 \mathrm{deg}^{2}$ using MeerKAT Phase 1 at $1.4 \mathrm{GHz}$ to a target rms flux density of $5 \mu \mathrm{Jy}$ beam $^{-1}$. This will allow detailed studies of the distant Universe and the evolution of the lower luminosity radio source populations into the EoR. Tier 2 will exploit the longer baselines of MeerKAT Phase 2 and increased bandwidth to observe a single $35 \mathrm{deg}^{2}$ pointing down to $1 \mu \mathrm{Jy}_{\text {beam }}{ }^{-1} \mathrm{rms}$. This will enable studies of the evolution of Milky Way-type galaxies up to $z \sim 4$ and pioneering weak lensing analyses at radio wavelengths. These observations will also be used to test techniques to increase dynamic range in preparation for the full SKA. Tier 3 will cover a much smaller area $\left(1 \mathrm{deg}^{2}\right)$ to $0.1 \mu \mathrm{Jy}_{\text {beam }}{ }^{-1} \mathrm{rms}$. This will allow us to isolate the level of AGN activity in SF galaxies and to investigate the morphological properties of AGN from $z \sim 0.5$ to $\sim 6$.

\subsection{Murchison Widefield Array}

The MWA (Lonsdale et al. 2009; Tingay et al. 2012) is a low-frequency synthesis telescope under construction at Boolardy, Western Australia, adjacent to ASKAP. It is due for completion in 2013, making it the first of the SKA precursor telescopes to commence full operation. It consists of 2048 dual-polarisation dipole antennas, arranged as 128 'tiles', each consisting of a $4 \times 4$ array of dipoles designed to operate in the $80-300 \mathrm{MHz}$ frequency range. Each tile performs an analogue beam-forming operation, narrowing the FOV to an electronically steerable $25^{\circ}$ at $150 \mathrm{MHz}$. The majority of the tiles (112) are scattered across a roughly 1.5$\mathrm{km}$ core region, forming an array with very high imaging quality, and an FOV of several hundred square degrees at a resolution of several arcmin. The remaining 16 tiles are located outside the core, yielding baselines up to $3 \mathrm{~km}$ to allow higher angular resolution. The MWA calibration and imaging is undertaken on 24 IBM iDataPlex dual Xeon servers, each housing 2 NVIDIA Tesla M2070 Graphics Processing Units. The correlated data are calibrated in real time using novel position-dependent self-calibration algorithms.

MWA is focused on four key science projects (Bowman et al. 2012): (a) the detection and characterisation of threedimensional brightness temperature fluctuations in the $21-\mathrm{cm}$ line of neutral hydrogen during the EoR at redshifts from 6 to 10 , (b) solar imaging and remote sensing of the inner heliosphere via propagation effects on signals from distant background sources, (c) high-sensitivity exploration of the variable radio sky, and (d) wide-field galactic and extragalactic astrophysics including a deep all-sky survey over the MWA frequency range with full polarimetry and spectral resolution. An initial survey of $2400 \mathrm{deg}^{2}$ on the prototype
'32T' system has recently been completed (Bernardi et al. 2012) and a full sky survey south of $+30^{\circ}$ is being planned for the full array.

\subsection{Other survey telescopes}

We acknowledge that many other radio telescopes, such as the Giant Metre Wave Radio Telescope (GMRT; Ananthakrishnan \& Pramesh Rao 2001) and the Very Long Baseline Array (VLBA; Napier et al. 1994), are also making very significant contributions both to the continuum survey science and to the survey techniques necessary for the SKA, but are outside the scope of this paper.

\section{SCIENCE GOALS}

\subsection{The radio sky}

The goal of most SKA pathfinder radio continuum surveys is to make a deep radio continuum survey of a significant fraction of the sky. For example, EMU and WODAN together will cover the entire sky at $1.4 \mathrm{GHz}$ to a depth of about $10-15 \mu \mathrm{Jy}$ beam $^{-1} \mathrm{rms}$ with an angular resolution of 10-15 arcsec. LOFAR will complement this with a lowfrequency survey of comparable intrinsic sensitivity, and VLA and MeerKAT will be able to probe individual objects, or survey smaller areas, to a much greater depth. The largest comparable survey currently existing is the benchmark NVSS survey (Condon et al. 1998), compared with which EMU plus WODAN will be about 40 times more sensitive, with an angular resolution about four times better, and with higher sensitivity to extended structures. EMU and WODAN together are expected to detect and catalogue about 100 million sources.

At the sensitivity level of these surveys, the radio sky has already been studied by a number of deep surveys, and the flux-density-dependent surface-density statistics have been extensively measured (Figure 3). However, these surveys cover a total of only a few square degrees (Hopkins et al. 2003; Huynh et al. 2005; Biggs \& Ivison 2006; Norris et al. 2006; Middelberg et al. 2008b; Miller et al. 2008; Schinnerer et al. 2007; Morrison et al. 2010; J. K. Banfield et al., in preparation; Guglielmino et al., in preparation; C. A. Hales et al., in preparation). We know broadly what types of galaxy we will detect, and their approximate redshift distribution (see Figure 4) but a number of significant questions remain:

- What types of object dominate the source counts at low fluxes?

- Are there undiscovered populations?

- What causes the scatter between different surveys at low fluxes?

- Do we need better simulations to guide research in this area?

- How do environment and large-scale structure affect the evolution of radio sources? 


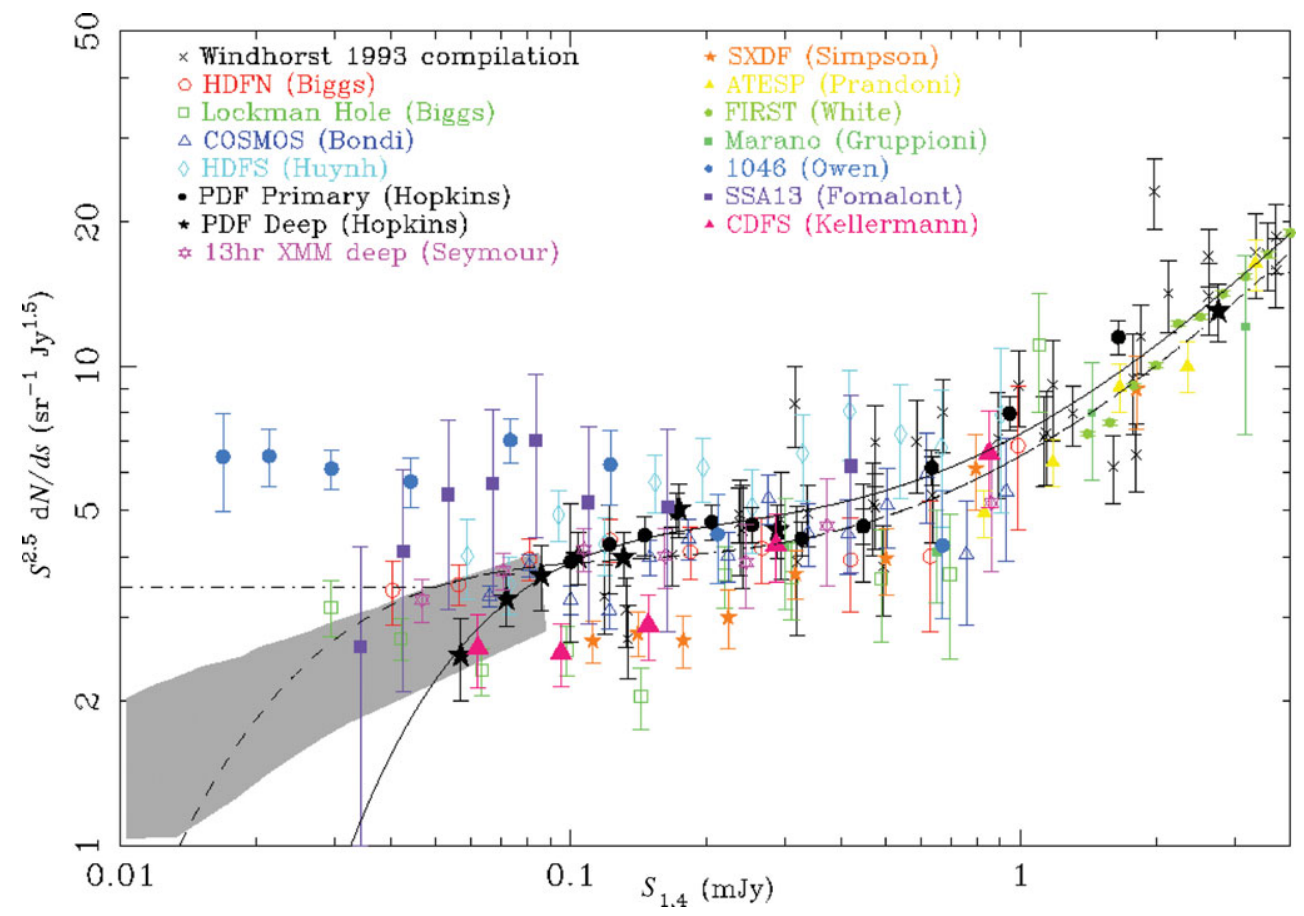

Figure 3. The Euclidean normalised differential radio source counts at $1.4 \mathrm{GHz}$, based on and updated from the distribution shown in Hopkins et al. (2003). The solid curve is the polynomial fit from Hopkins et al. (2003), and the dashed curve is an updated polynomial fit. The horizontal dot-dashed line represents a non-evolving population in a Euclidean universe. The shaded region shows the prediction based on fluctuations due to weak confusing sources ( a 'P(D) analysis') from Condon (1974) and Mitchell \& Condon (1985).

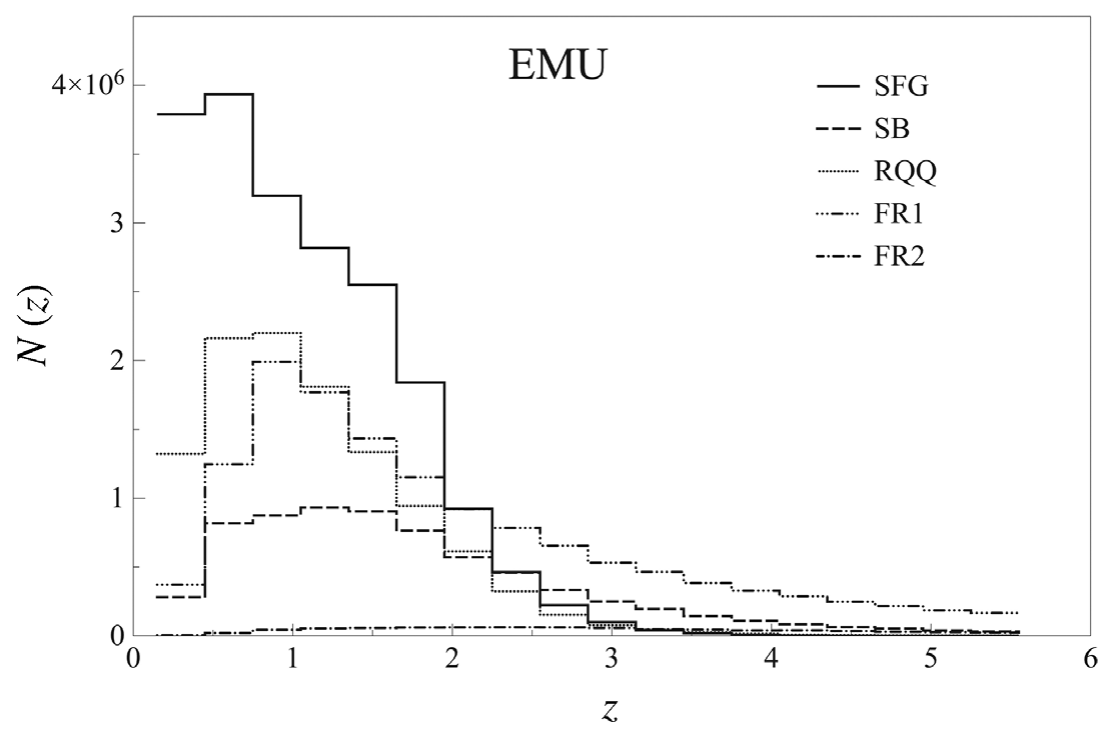

Figure 4. Expected redshift distribution of sources with $S_{1.4}>10 \mu \mathrm{Jy}$ beam $^{-1}$, based on the SKADS simulations (Wilman et al. 2008, 2010). The five lines show the distributions for SF galaxies (SFG), starburst galaxies (SB), radio-quiet quasars (RQQ), and radio-loud galaxies of Fanaroff-Riley types I and II (FRI and FR2; Fanaroff \& Riley 1974). The vertical scale shows the total number of sources expected to be detected.

At high flux densities, the source counts (Figure 3) are dominated by AGNs, following a smooth power-law distribution from Jansky levels down to about $1 \mathrm{mJy}$. Below $1 \mathrm{mJy}$, the normalised source counts flatten, suggesting an additional population of radio sources. The source counts below $1 \mathrm{mJy}$ are less well constrained, with a scatter between different surveys which can reach a factor of $\sim 2-3$.

While some fraction of this scatter may be due to cosmic variance (e.g. I. Prandoni et al., in preparation), that cannot be the only cause. For comparison, in the X-ray band, the 


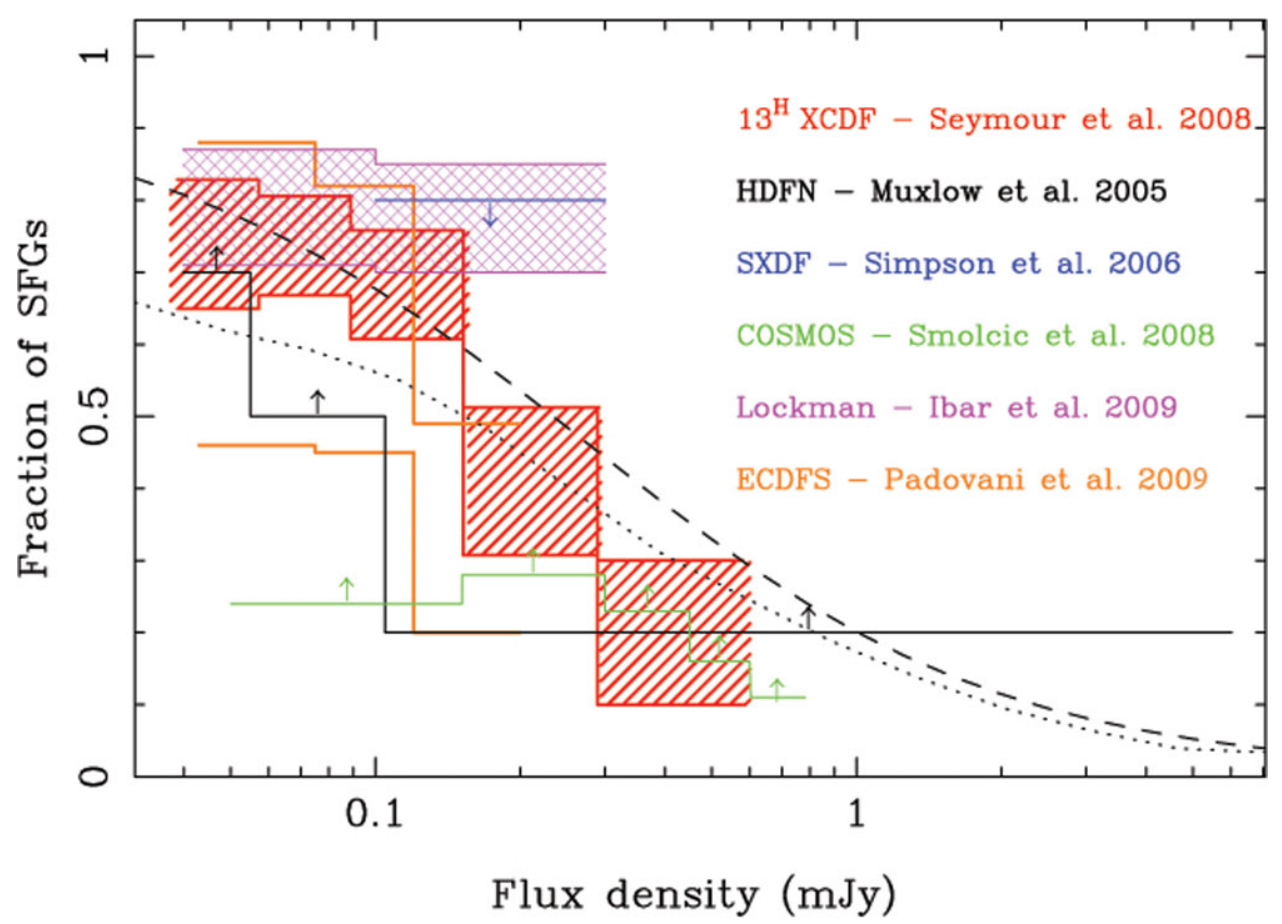

Figure 5. Differential fraction of SF galaxies as a function of 1.4-GHz flux density, taken from Norris et al. (2011b). Shaded boxes, and the two lines for Padovani et al., show the range of uncertainty in the survey results. Arrows indicate constraints from other surveys. These results show that the fraction of SF galaxies increases rapidly below $1 \mathrm{mJy}$ and, at the $50 \mu \mathrm{Jy}$ survey limit of EMU/WODAN, about $75 \%$ of sources will be SF galaxies.

scatter between the GOODS North and South fields is only $\sim 25 \%(\sim 3 \sigma)$. Furthermore, Condon et al. (2012) have shown that some of the largest variations can be accounted for by errors in the many necessary corrections for effects such as resolution bias, clean bias, Eddington bias, and completeness corrections, together with imaging errors such as excessive deconvolution, bandwidth smearing, and insufficient beam sampling in the image plane. There is also an inconsistency in that some authors measure the numbers of radio components, while other measure the numbers of sources, each of which may consist of several components, the numbers of which in turn may vary as a function of flux density.

Resolving this discrepancy is critical, since this factor of 2-3 scatter introduces large uncertainties in the comparison of observed number counts with detailed, model-based predictions. Obviously, radio surveys need to move beyond the small-area surveys that have previously dominated the literature. It is also essential that, before the large radio surveys start, we obtain consensus on how to image and analyse radio survey data to produce reliable and consistent source flux densities and source counts.

\subsubsection{The radio sky at $\mu J y$ levels: AGN or star formation?}

After years of intense debate, it is now clear that while SF galaxies contribute significantly to the sub-mJy counts, they do not dominate it above $\sim 200 \mu \mathrm{Jy}$. Recent results (e.g. Seymour et al. 2008; Smolčić et al. 2008; Padovani et al. 2009), which are summarised in Figure 5, show that both SF galaxies and AGNs contribute significantly to the source counts. About half of these AGNs are radio-quiet, characterised by relatively low radio-to-optical flux density ratios and radio powers, as compared with radio quasars (see also Prandoni 2010). Above $1 \mathrm{mJy}$, the contribution of radio-quiet objects to the source counts is insignificant, and they are dominated by radio-loud AGNs.

To probe the radio-quiet AGN component, it is therefore necessary to analyse the deepest radio fields $(S \leq$ $100-200 \mu \mathrm{Jy}$ ), where most sources are typically associated with SF galaxies. Radio-quiet AGNs share many properties with SF galaxies: they have similar radio luminosities $\left(10^{22}-10^{24} \mathrm{~W} \mathrm{~Hz}^{-1}\right)$, steep radio spectra $(\alpha<-0.5)$, and similar infrared/radio flux ratios (e.g. Roy et al. 1998).

In addition, radio-quiet AGNs are often associated with faint optical galaxies characterised by Seyfert-2-like spectra, containing both SF and AGN components. Even when available, if optical spectroscopy is not of sufficient quality, it can be difficult to derive reliable emission line ratios, and distinguish between starburst and Seyfert 2 spectra, through BPT (Baldwin, Phillips, \& Terlevich 1981) diagrams (see e.g. Prandoni et al. 2009). This makes it difficult to distinguish radio-quiet AGNs from SF galaxies, even with multiwave length information, although the availability of X-ray data can help (Padovani et al. 2011).

Spitzer IRAC colours, when available, can be very effective in separating radio-quiet AGNs from SF galaxies as 


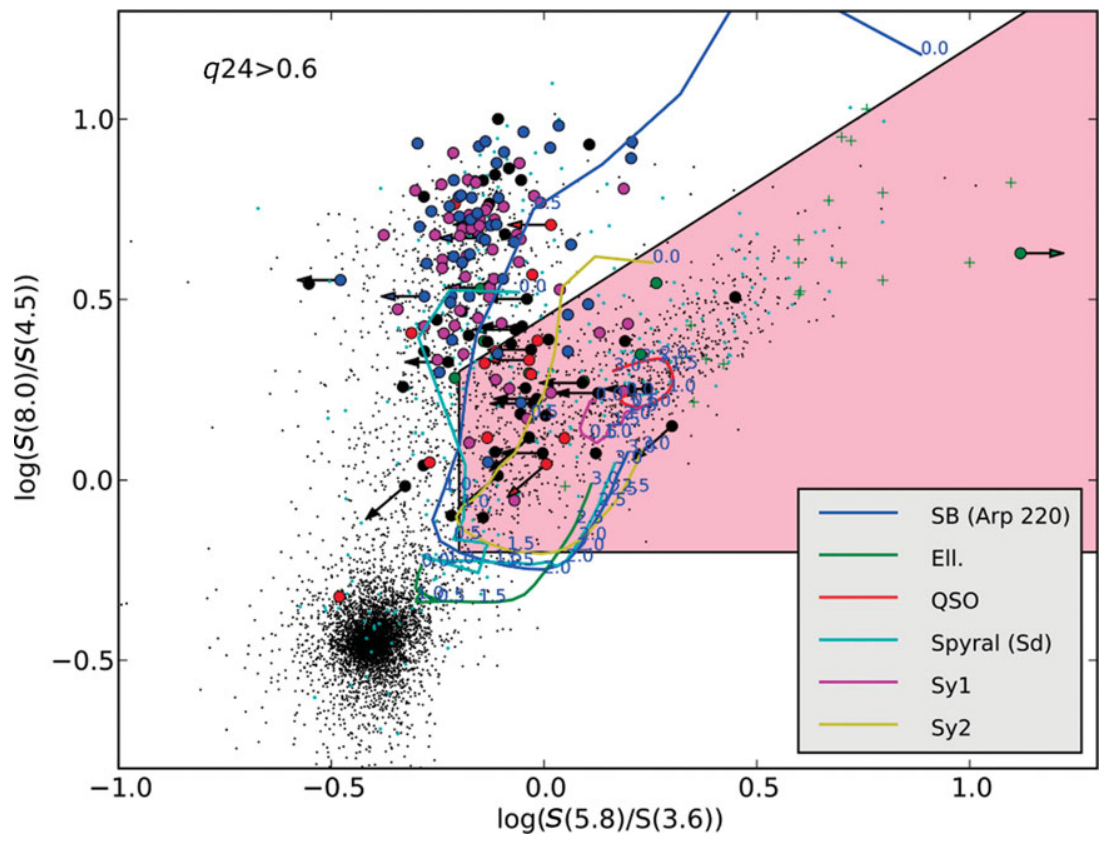

Figure 6. IRAC colour-colour plot of the FLS radio sources with $q_{24}=\log \left(S_{24 \mu} / S_{1.4 \mathrm{GHz}}>0.6\right.$ (filled symbols), from Prandoni et al. (2009). Colours refer to optical spectral classification: SF galaxies (blue); narrow/broad line AGNs (green); early-type galaxies (red); galaxies with narrow emission line, which do not have a secure optical classification (magenta); sources with no optical spectroscopy available (black). Arrows indicate upper/lower limits. The lines indicate the expected IRAC colours as a function of redshift for different source types (see legend). The expected location for AGNs is highlighted in pink. For reference, we also show IRAC colours of (a) all FLS IRACidentified radio sources (no optical identification selection applied, cyan dots); (b) the entire FLS IR-selected star/galaxy population (no radio selection applied, black dots); and (c) a sample of high-redshift obscured (type-2) quasars (Martinez-Sansigre et al. 2006), green crosses.

shown in Figure 6, where the well-known IRAC colourcolour plot (Lacy et al. 2004) is exploited for the First Look Survey (FLS) deep radio field. A fraction of sources with typical AGN IRAC colours appear to be genuine radio-quiet AGNs, and account for $\sim 45 \%$ of the overall AGN component in the FLS.

A number of other methods can also be used to distinguish AGNs from SF galaxies in radio surveys (e.g. Norris, Middelberg, \& Boyle 2008), including

- radio morphology (e.g. Biggs \& Ivison 2008; Biggs, Younger \& Ivison 2010),

- radio spectral index (e.g. Ibar et al. 2009, 2010),

- radio-far-infrared ratio (e.g. Norris et al. 2006; Middelberg et al. 2008b),

- radio-near-infrared ratio (e.g. Willott et al. 2003),

- radio polarisation (e.g. C. A. Hales et al., in preparation),

- radio variability (e.g. Norris et al. 2008; Chatterjee et al. 2010; Murphy et al. 2012),

- optical and IR colours, including the use of spectral energy distribution (SED) templates (e.g. Lacy et al. 2004),

- optical line ratios (e.g. Baldwin et al. 1981),

- X-ray hardness ratio (e.g. Rosati et al. 2002),

- X-ray power (e.g. Padovani et al. 2011),
- radio source brightness measured by VLBI (e.g. Kewley et al. 2000; Parra et al. 2010; Alexandroff et al. 2012).

None of these techniques is foolproof and universal, and a combination of techniques is necessary to provide unambiguous classification. In preparation for the daunting task of classifying tens of millions of radio sources, we need to define reliable methods for classifying radio sources.

\subsubsection{Composite galaxies}

It is well established (e.g. Roy et al. 1998) that the radio emission from Seyfert galaxies contains significant contributions from both AGN and SF activity, and there is growing recognition (Norris et al. 2008; Lutz et al. 2010; Shao et al. 2010; Seymour et al. 2011) that this may also be true of high-luminosity galaxies, particularly at high redshift. Many of these galaxies are not simply 'star-forming' or 'AGNs' but include a significant contribution from both. In extreme cases (Norris et al. 2012), a galaxy may appear to be 'pure AGN' at one wavelength and 'pure SF' at another.

Particularly at high redshifts, such composite AGN/SF systems constitute a significant fraction of radio sources, and so a simple classification into AGN or SF galaxy is inadequate. Instead, the contribution to the galaxy's luminosity from both AGN and SF activity must be assessed, to measure 
the relative contributions from underlying physical properties such as black hole and galaxy mass, star-formation rate (SFR), environment, etc. However, the relative contribution will vary depending on the observing band, and so comprehensive multiwavelength data are required. However, such detailed information will be hard to obtain for very large samples, perhaps necessitating a simpler classification scheme, which may lead to oversimplifications or even to incorrect interpretation of the data.

It is therefore essential to develop methods which fit the SED of a given radio source with both a starburst and AGN component (e.g. Afonso et al. 2001). This approach is clearly only possible for fields with good multiwavelength data, but will inform analysis of fields with lower quality ancillary data. Observations from Spitzer and Herschel cover the bulk of the energy output of most galaxies and can be used to distinguish the starburst and AGN components. For example, the far-IR is directly related to the SFR, and, if the FIRradio correlation remains true at all redshifts (e.g. Mao et al. $2011 \mathrm{a}$, and references therein), can be used to determine what fraction of the radio emission is due to star formation. Hence, the remaining radio emission would be due to AGN activity. The AGN bolometric luminosity can be estimated from the mid-IR component which is not necessarily proportional to the AGN emission at radio wavelengths. Once the starburst and AGN fractional contribution to individual sources are determined, it is possible to construct derived results such as luminosity functions and relative contributions by type to the source counts.

\subsubsection{Are there undiscovered populations of radio sources?}

The cosmic radio background (CRB) has been well studied at high frequencies by instruments such as COBE and WMAP, but is less well studied at low frequencies.

Fixsen et al. (2011) measured the background sky temperature at five frequencies between 3 and $90 \mathrm{GHz}$ using an in situ calibrator on board the balloon-borne $A R C A D E 2$ experiment. These authors found an excess radio sky temperature above that due to the cosmic microwave background (CMB) below $10 \mathrm{GHz}$. The CRB was measured to have an excess of $50 \pm 7 \mathrm{mK}$ at $3.3 \mathrm{GHz}$ above a CMB temperature of $2.730 \pm$ $0.004 \mathrm{~K}$, which is a factor of five higher than expected from known source populations.

The ARCADE2 result is either due to an instrumental or calibration problem (e.g. incorrectly subtracting the Galactic foreground emission), or it is a startling result which will necessitate a drastic revision of our models of extragalactic radio sources. Given that Fixsen et al. (2011) appear to have taken careful steps to avoid errors, there is a prima facie case that this result is correct, and the radio-astronomical community has been galvanised to search for this putative new population. Since it is inconsistent with known radio source populations (e.g. De Zotti et al. 2010; Padovani 2011), it must, if confirmed, be caused by another population. For example, the radio emission could be caused by dark mat- ter annihilation (Fornengo et al. 2011), in which case the emission would trace the dark matter distribution of cluster galaxies, resulting in a scale size of $\sim$ arcmin. Other mechanisms are also possible, such as diffuse emission from clusters or haloes, or very low surface brightness emission from extended AGN radio lobes, or from a population of dwarf galaxies.

Condon et al. (2012) have conducted a deep survey with the VLA which shows that the $A R C A D E 2$ result cannot be caused by a population of compact objects spatially associated with galaxies. It is therefore either due to a diffuse population of objects which cannot be detected with the shortest baselines of the VLA observations, or they are due to an instrumental or calibration error. A number of observational programs continue with the VLA and ATCA to determine the cause of this result.

\subsubsection{Simulations}

There is widespread agreement that the SKADS continuum sky (Wilman et al. 2008) represents the current state of the art in simulations of the radio sky. Such simulations have two distinct functions.

- They represent useful approximations to the real sky which can be used to test algorithms and design surveys.

- They can be viewed as an attempt to encapsulate the current 'best knowledge' of the sky, and can therefore be used to test models of radio source evolution.

These two functions have different implications for future updates of the SKADS continuum sky. For example, the SKADS continuum sky was constructed from the best information then available of the radio luminosity functions (RLFs) of different populations of radio objects, and deeper and wider surveys have since become available. The SKADS simulation also assumes that sources are members of distinct populations, omitting the composite sources in which both star formation and AGN activity contribute to their radio luminosity. Such small differences from the real sky are unlikely to be important for the design of a survey, but will be important if SKADS is to be used to test theoretical models.

A useful, and relatively simple, modification to the SKADS continuum sky would be to change the assumed luminosity function for one or more of the populations in order to assess how that might influence the results of various continuum surveys. This change might be implemented in a parametric way, allowing users to specify parameters of a luminosity function.

More difficult would be an attempt to incorporate 'best knowledge', with the aim of producing 'realistic' sky images. Experience from the Herschel mission bolsters the concern that identifying and incorporating information can be extremely difficult. Similarly, incorporating polarisation information would be useful, so that one could calculate Faraday RMs, but would be quite difficult in practice because of the need to trace the geodesic of a photon in order to determine 
the RM. Nevertheless, there are efforts underway to insert realistic source shapes into the SKADS sky, and the SKADS team is investigating how to add the signature of the cosmic dipole.

It would be very useful to include models of more complex structures in the SKADS sky, such as those of large radio sources, diffuse cluster sources, and structures near the Galactic plane, which could potentially limit the lowest Galactic latitude to which an 'all-sky' survey could probe. However, as described in Section 4.3, imaging a field containing many compact sources may have similar issues as to imaging a field containing large-scale structure.

\subsection{The evolution of star formation}

The cosmic star formation history has been studied thoroughly over the past decade and a half, building up a remarkably consistent picture. As shown in Figure 5, only a small fraction of radio sources above $1 \mathrm{mJy}$ are SF galaxies. Below $1 \mathrm{mJy}$, a much larger volume of the SF galaxy population becomes detectable, and the population becomes significant at flux densities below about $0.2 \mathrm{mJy}$. SF galaxies will therefore dominate the deep and wide area continuum surveys proposed with the ASKAP, APERTIF, and MeerKAT telescopes, probing the entire sky to depths of about $50 \mu \mathrm{Jy} \mathrm{beam}^{-1}$.

The star formation history is now well established up to almost the EoR. The space density of SFR declines by an order of magnitude between a redshift of unity and zero, and is almost flat at higher redshifts, with the suggestion of a decline above a redshift of $z \sim 5$ (Hopkins \& Beacom 2006). At higher redshifts there is still some discrepancy, with evidence from Lyman dropout sources suggesting a dramatic decline above $z=6$ (Bouwens et al. 2008), and evidence from gamma-ray bursts suggesting a much slower decline (Kistler et al. 2009). The mass dependence of the SFR density has also begun to be constrained, with the contribution of more massive galaxies to the SFR density increasing at higher redshift (e.g. Juneau et al. 2005; Feulner et al. 2005; Mobasher et al. 2009). Environmental effects as well have been shown to change with redshift, with star formation in high density, cluster like environments being enhanced at early times (Elbaz et al. 2007) but suppressed at later times (Lewis et al. 2002; Gomez et al. 2003).

Together these lines of evidence point to a picture where star formation at the earliest times is dominated by massive systems in the densest regions, which rapidly exhaust their fuel, ultimately becoming the progenitors of today's brightest cluster galaxies and cD galaxies. At later epochs, the star formation is dominated by somewhat lower mass systems in somewhat less dense environments, similar to cluster outskirts, which in turn exhaust their fuel, until at the lowest redshifts the star formation is dominated by low-mass galaxies living in the least dense environments (Figure 7). This picture is still simplistic, and omits significant details such as how the evolving luminosity function of SF galaxies changes with galaxy mass and environment, and whether and how the

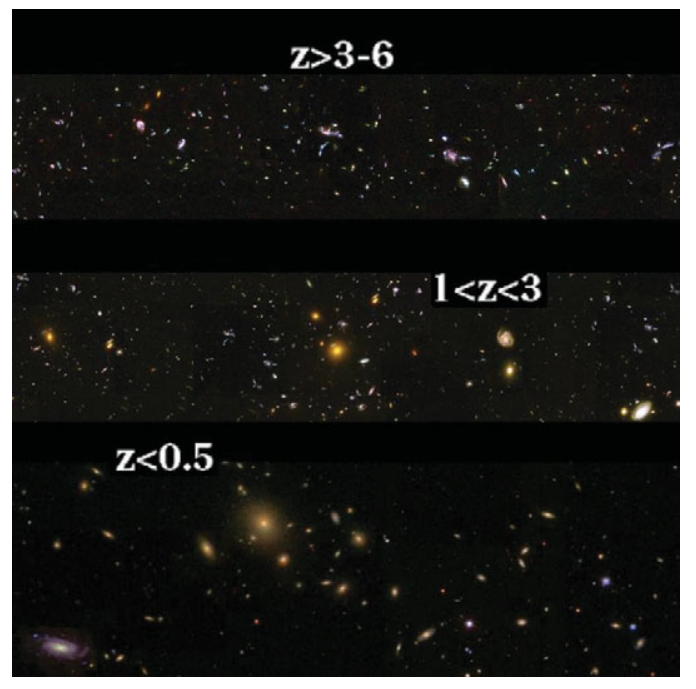

Figure 7. An illustration of the density dependence of the downsizing of galaxy SFRs, using optical images from a variety of sources. At the highest redshifts $(z>3)$, star formation is occurring predominantly in massive galaxies (those that become local massive ellipticals) that live in the most overdense regions (that evolve into today's massive clusters). At lower redshifts $(1<z<3)$, where EMU/WODAN will be sensitive to the most extreme SF systems, star formation is dominated by lower mass systems, in less dense environments. By the current epoch, star formation is limited primarily to low-mass galaxies in the outskirts of clusters and in the lowest-density environments.

stellar initial mass function varies (e.g. Gunawardhana et al. 2011).

\subsubsection{Measuring star formation rates from radio observations}

At redshifts between $1 \lessgtr z \lessgtr 3$, when galaxies were undergoing rapid evolution, the star formation activity appears to have been dominated by dusty, heavily obscured, starbursting galaxies (e.g. Chary \& Elbaz 2001; Caputi et al. 2007; Magnelli et al. 2010; Murphy et al. 2011). Optical and nearinfrared observations are therefore seriously hampered by dust extinction. Thus, deep radio continuum surveys provide an important tool for measuring the cosmic star formation history of the Universe.

A key result from the next generation of wide and deep radio surveys will therefore be the measurement of the radio luminosity due to star formation in a wide range of galaxies. Converting this radio luminosity to a SFR depends on a conversion factor which is principally based on the infrared/radio correlation (Yun et al. 2001; Bell 2003; Seymour et al. 2008). However, this correlation has not yet been well determined at the high end of the luminosity function, since the number of ultraluminous IR galaxies (ULIRGs) in these papers is very small, or at high redshifts, although evidence so far suggests the correlation remains constant (Mao et al. 2011a).

At high redshifts, however, we know that the comoving SFR density is increasingly dominated by more luminous galaxies (with naturally higher SFRs). In Figure 8, we show the comoving infrared luminosity density, a proxy for the 


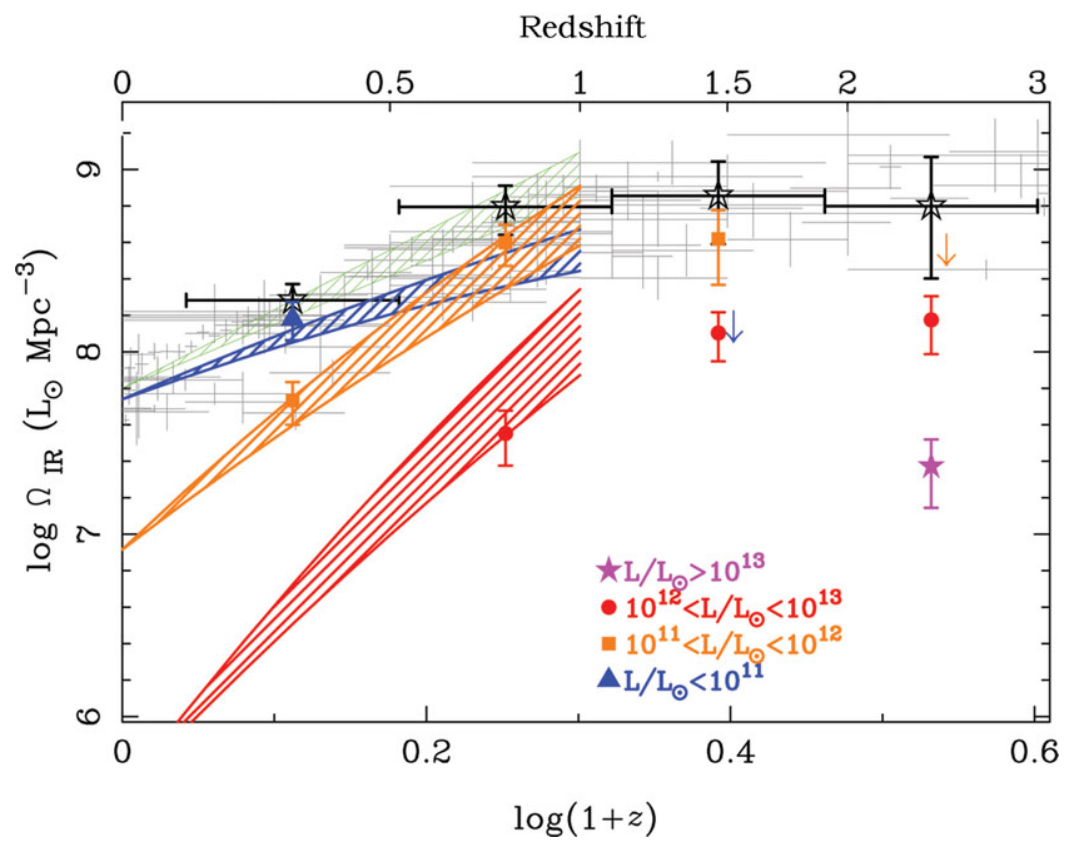

Figure 8. The comoving infrared luminosity density of SF galaxies as a function of redshift, separated into four infrared luminosity ranges. The shaded regions are from Le Floc'h et al. (2005) and the bold points are derived from the analysis of faint radio sources in the $13 \mathrm{H}$ field (Seymour et al. 2008) where the SF radio luminosities have been converted to infrared luminosities using the relation of Bell (2003). The faint, grey points are from the compilation of Hopkins \& Beacom (2006) converted to IR luminosity density. The figure shows that ULIRGs make an increasing contribution to the total star formation budget above redshifts of unity.

comoving SFR density, as a function of redshift. We also separate the contribution by infrared luminosity and find that ULIRGs represent an increasing fraction of the total star formation budget above a redshift of unity. Hence, if we are to use deep radio surveys to probe the star formation history of the Universe at high redshift, we must obtain a considerably more accurate conversion of the radio luminosity to SFR for galaxies with high SFRs. This conversion factor could also depend upon other parameters such as stellar mass, environment, and metallicity.

\subsubsection{Measuring SFRs at $10 \mathrm{GHz}$}

Radio continuum emission from galaxies typically arises from two processes that are both tied to the massive SFR. At low frequencies (e.g. $\$ 2 \mathrm{GHz}$ ), the radio continuum is dominated by non-thermal synchrotron emission arising from cosmic-ray (CR) electrons that have been accelerated by shocks from supernova remnants and are propagating through a galaxy's magnetised ISM. This physical link to massive star formation provides the foundation for the far-infrared (FIR)-radio correlation (e.g. Helou, Soifer, \& Rowan-Robinson 1985; Condon 1992; Yun et al. 2001; Murphy et al. 2006). However, this link is not at all direct given that there are a large number of physical processes affecting the propagation of CR electrons and the heating of dust (e.g. CR diffusion, magnetic field strength/structure, dust grain sizes and composition) that must conspire together to keep this relation intact (Bell 2003).
High frequency $(\sim 10-100 \mathrm{GHz})$ radio emission, on the other hand, offers a relatively clean way to quantify the current star formation activity in galaxies. At these frequencies, emission is generally optically thin and dominated by freefree radiation, which is directly proportional to the ionising photon rate of young, massive stars. While this picture could be complicated by the presence of anomalous dust emission (e.g. Kogut et al. 1996; de Oliveira-Costa et al. 1997; Leitch et al. 1997), which occurs at these frequencies and is thought to arise from spinning dust grains (e.g. Erickson 1957; Draine \& Lazarian 1998), it is currently unclear whether this component contributes significantly to globally integrated measurements (Murphy et al. 2010). Thus, higher frequency radio observations may be particularly powerful for precisely measuring the star formation history of the Universe unbiased by dust.

For example, surveys at $v \gtrsim 10 \mathrm{GHz}$ start to probe the rest-frame $v \gtrsim 30 \mathrm{GHz}$ emission from SF galaxies by $z \gtrsim 2$ for which $\gtrsim 50 \%$ of the emission is thermal (free-free). This is illustrated in Figure 9, where the thermal fraction of the observed 10- and 1.4-GHz emission is shown against redshift for SF galaxies having intrinsic magnetic field strengths of 10,50 , and $100 \mu \mathrm{G}$ while still obeying the FIR-radio correlation at $z=0$. In addition to measuring higher restframe frequencies with increasing redshift, this calculation also accounts for the suppression of a galaxy's non-thermal emission due to rapid cooling of CR electrons from inverse Compton (IC) scattering off the CMB, whose radiation field 


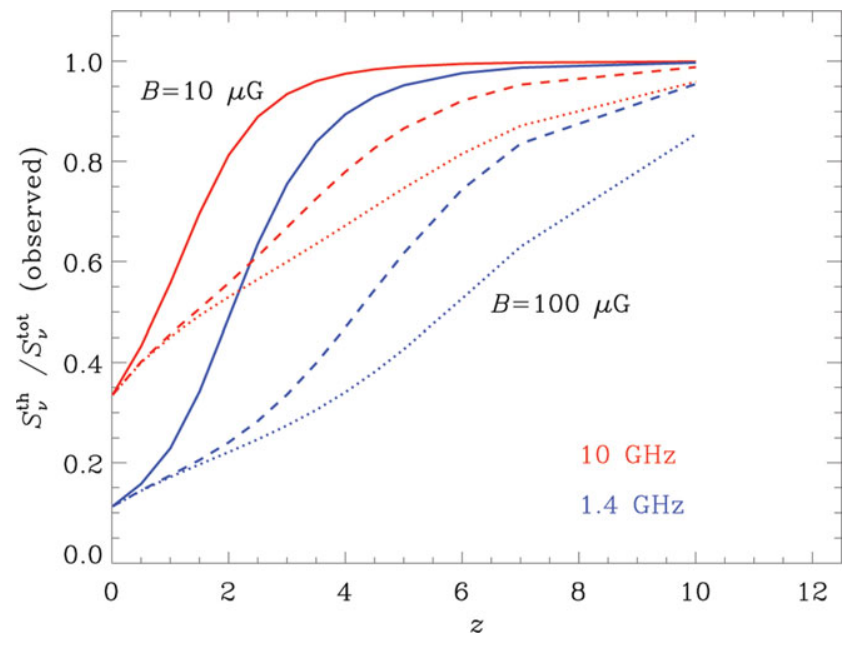

Figure 9. The fraction of radio emission of SFG due to thermal processes as a function of redshift. Galaxy magnetic fields of 10 (solid lines), 50 (dashed lines), and $100 \mu \mathrm{G}$ (dotted lines) are shown. The radio emission from observations at $10 \mathrm{GHz}$ is dominated by free-free processes beyond a redshift of $z \gtrsim 2$ even for magnetic field strengths of $\sim 100 \mu \mathrm{G}$, making it an ideal measure for the current SFR of high- $z$ galaxies (Murphy 2009).

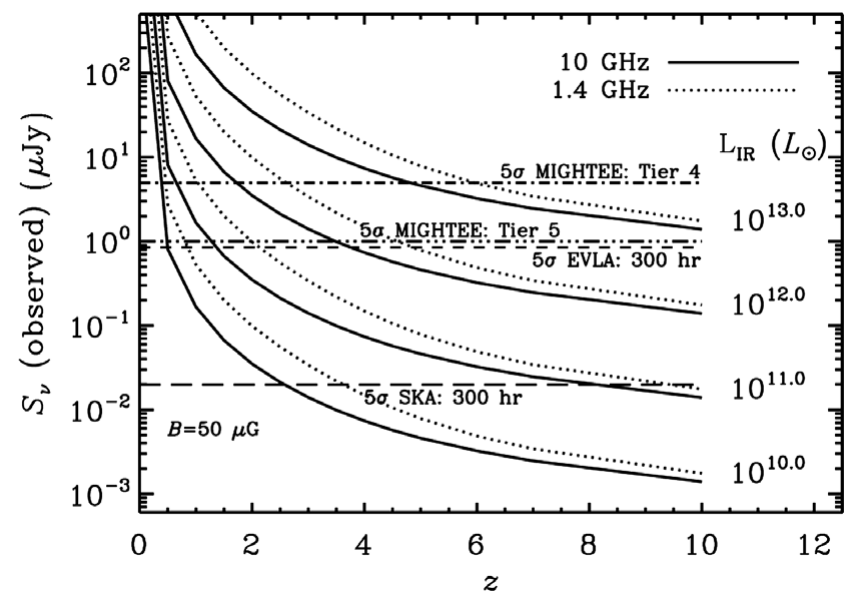

Figure 10. The expected $10 \mathrm{GHz}$ (solid line) and $1.4 \mathrm{GHz}$ (dotted line) flux densities for galaxies of different IR luminosities assuming an intrinsic magnetic field strength of $50 \mu \mathrm{G}$. The depths of possible future surveys taken by the VLA, MeerKAT (MIGHTEE), and SKA are shown. Since the non-thermal emission from SF galaxies should be suppressed by increased IC scattering of CR electrons off of the CMB, the discrepancy between the point source sensitivity requirements of surveys at 1.4 and $10 \mathrm{GHz}$ falls below a factor of $\sim 2$ by $z \gtrsim 4$.

energy density scales roughly as $U_{\mathrm{CMB}} \sim(1+z)^{4}$ (Murphy 2009). By $z \gtrsim 4$, nearly $80 \%$ of observed $10-\mathrm{GHz}$ radio continuum is due to free-free emission for a galaxy having a large (i.e. $50 \mu \mathrm{G}$ ) magnetic field.

Additionally, we can compare the relative sensitivity requirements of surveys at 10 and $1.4 \mathrm{GHz}$ to detect cosmologically important galaxies at early epochs.

This is shown in Figure 10, where we show the expected 10- and 1.4-GHz flux densities from galaxies having a range in IR $(8-1000 \mu \mathrm{m})$ luminosities and an intrinsic magnetic field of $50 \mu \mathrm{G}$. This figure essentially shows the selection function of radio surveys to SF galaxy populations as a function of redshift. While higher frequency surveys need to be much more sensitive to detect the same luminosity class of galaxies at $1.4 \mathrm{GHz}$, this becomes less of a problem at higher redshifts. For example, by $z \gtrsim 4$, the sensitivity requirement of a $10-\mathrm{GHz}$ survey is less than a factor of $\sim 2$ deeper than a corresponding 1.4-GHz survey to detect the same population of SF systems. For an intrinsic magnetic field of $10 \mu \mathrm{G}$, this small discrepancy between point source sensitivity requirements for 10 - and 1.4-GHz surveys occurs at a $z \gtrsim 2$. Given that high-frequency observations are almost as sensitive to high- $z$ SF galaxies as low-frequency observations, and that higher frequency observations provide a much more robust measure of star formation activity, surveys at $v \gtrsim 10 \mathrm{GHz}$ will be invaluable for accurately tracing the star formation history of the Universe.

Figure 10 also shows the expected depths from potential continuum surveys using the VLA, MeerKAT, and SKA. The tentative Tier 4 and 5 steps of the MIGHTEE survey plan to map 0.25 and $0.01 \mathrm{deg}^{2}$ down to an rms of 1 and $0.2 \mu \mathrm{Jy}$ at $12 \mathrm{GHz}$, respectively. Although the precise area on the sky has not yet been defined, obvious targets are the Chandra Deep Field-South and COSMOS fields. While the depth of a megasecond exposure is shown for the VLA, the area of such a survey would be restricted by the small primary beam at $10 \mathrm{GHz}\left(\theta_{\mathrm{PB}} \approx 4-5\right.$ arcmin). However, VLA surveys reaching $\mu \mathrm{Jy}$ depths for entire fields such as GOODS-N at 10 $\mathrm{GHz}$ have already been proposed, allowing synergy with future ALMA observations. Clearly, the SKA will revolutionise any such high-frequency continuum surveys using either the VLA or MeerKAT by being $\gtrsim 2$ orders of magnitude deeper for the same integration times.

\subsection{Evolution of AGNs}

\subsubsection{The AGN component in deep radio fields}

Radio source counts at $\mu \mathrm{Jy}$ levels are dominated by SF galaxies, but their contribution decreases rapidly with increasing flux density (e.g. Seymour et al. 2008), and AGNs contribute significantly at radio fluxes below $1 \mathrm{mJy}$ (e.g. Gruppioni et al. 1999; Georgakakis et al. 1999; Magliocchetti et al. 2000; Prandoni et al. 2001; Afonso et al. 2006; Norris et al. 2006; Mignano et al. 2008; Smolčić et al. 2008; Padovani et al. 2009). Recent estimates of their relative contributions are summarised in Figure 5.

Models of the sub-mJy radio population (e.g. Wilman et al. 2008) therefore include three main components:

- SF galaxies,

- The extrapolation to low flux densities of the classical radio-loud AGN population (radio galaxies and radioQSOs),

- A radio-quiet (or low-luminosity) AGN component. 
The unexpected presence of large numbers of AGN-type sources at sub-mJy levels implies a large population of low/intermediate power AGNs, which may have important implications for the black hole-accretion history of the Universe. It will be particularly important to determine the accretion mode (see Section 3.3.2) of these low-power AGNs.

Radio and optical studies of the Australia Telescope ESO Slice Project (ATESP) sub-mJy sample (Prandoni et al. 2006; Mignano et al. 2008) have shown that at fluxes $0.4<S<1$ mJy, a prominent class of low-luminosity $\left(P<10^{24} \mathrm{~W} \mathrm{~Hz}^{-1}\right)$ AGNs associated with early-type galaxies appears. These sources have typically flat or inverted radio spectra $(\alpha>-$ $0.5)$ and compact sizes $(d<10-30 \mathrm{kpc})$. This source class produces a flattening of the average spectral index at flux densities around $0.5-1 \mathrm{mJy}$. A similar flattening has been found also in deep low-frequency $(350 \mathrm{MHz}$ ) surveys (Owen et al. 2009; Prandoni et al. 2011). Radio spectral index studies (e.g. Prandoni et al. 2010) suggest that this class of objects is presumably associated with core-dominated self-absorbed FRIlike radio galaxies, perhaps representing a low-luminosity extension of the classical radio-loud radio galaxy population, which dominates at flux densities $S \gg 1 \mathrm{mJy}$. On the other hand, deeper radio fields have shown that flat/inverted radio spectra become less frequent at lower flux densities (100 $<S<400 \mu$ Jy) (Owen et al. 2009; Prandoni et al. 2009), where steep-spectrum SF galaxies and radio-quiet AGNs are increasingly important.

Our current understanding of the AGN component in deep radio fields can be summarised as follows:

- above flux densities $S \sim 400 \mu \mathrm{Jy}$, there is no clear evidence for a radio-quiet AGN component;

- sub-mJy radio-loud AGNs are mostly low-power, compact (self-absorbed) jet-dominated systems, and seem to be a low power counterpart of the classical bright radio galaxy population;

- at lower flux densities, radio-quiet AGNs become increasingly important and at $S \sim 100 \mu \mathrm{Jy}$ they account for about $45 \%$ of the overall AGN component;

- radio-quiet AGNs show radio/optical/IR properties consistent with radio follow-up of optically selected radioquiet AGNs (Kukula et al. 1998), compact radio sizes, steep radio spectra, luminosities of $P<10^{24} \mathrm{~W} \mathrm{~Hz}^{-1}$, and Seyfert-2 optical spectra and/or mid-IR colours.

Multiwavelength analyses of larger and deeper radio samples are needed to confirm these results, and to obtain quantitative constraints for evolutionary modelling of faint AGNs. We can expect this field to move forward significantly in the next years, thanks to the combination of wide-field and allsky surveys (sampling large local volumes) and very deep fields (sampling low powers at high redshifts) planned with the facilities described in this paper. An example of what can be obtained is illustrated in Figure 11, where we show the AGN luminosity function at different redshifts that can be derived from the combination of EMU and MIGHTEE.

\subsubsection{Accretion modes}

AGN activity, and associated black-hole growth, occurs in at least two different modes, each of which may have an associated feedback effect upon the AGN host galaxy:

(i) A fast accretion mode (often referred to as cold-mode accretion or quasar-mode accretion) associated with quasars (Silk \& Rees 1998); this radiatively efficient accretion mode may be important in curtailing star formation at high redshifts and setting up the tight relationship between black hole and bulge masses observed in the nearby Universe (e.g. Magorrian et al. 1998).

(ii) A radiatively inefficient slow accretion mode (often referred to as hot-mode accretion or radio-mode accretion) (Croton et al. 2006; Bower et al. 2006), the observational manifestation of which is low-luminosity radio sources; this mode is thought to be responsible for maintaining elliptical galaxies at lower redshifts as 'old, red and dead' (e.g. Best et al. 2006) and for preventing strong cooling flows in galaxy clusters (e.g. Fabian et al. 2003).

One of the most fundamental issues in understanding the role of AGNs in galaxy formation is the need to measure accurately the cosmic evolution of quasar activity and the accretion history of the Universe, and to compare this with the build-up of the stellar populations of galaxies. Do black holes and their host galaxies grow coevally, or does one precede the other? What is the primary mode of black hole growth?

Much of the growth of black holes is believed to occur in an obscured phase, and these 'Type-2' AGNs are difficult to identify. Even the deepest current X-ray observations do not detect the most heavily absorbed sources which cosmic Xray background synthesis models predict exist in abundance (e.g. Gilli et al. 2007), implying that much high- $z$ quasar activity has yet to be detected directly. These 'radio-quiet' quasars are not radio-silent, and their radio luminosity distribution peaks at about $L_{1.4 \mathrm{GHz}} \approx 10^{23} \mathrm{~W} \mathrm{~Hz}^{-1}$ (e.g. Cirasuolo et al. 2003). Deep radio surveys with the SKA pathfinders therefore offer an alternative route to identifying these distant AGNs, in a manner unbiased by dust and gas absorption at other wavelengths. Combined with multiwavelength data sets to separate source populations, the relative contribution of radio-quiet AGNs to the faint radio source population can be determined. This will enable investigation of the dependence of the fraction of obscured AGNs on luminosity and cosmic epoch, and thus the history of radiatively efficient accretion in the Universe to be determined.

The deep and wide radio surveys will also allow study of the role of low-luminosity radio sources in galaxy evolution. The low-luminosity radio-AGN population is dominated by a population of sources in which there is little evidence for radiative emission from an accretion disc, and the bulk of the accretion power is channelled into the expanding radio 


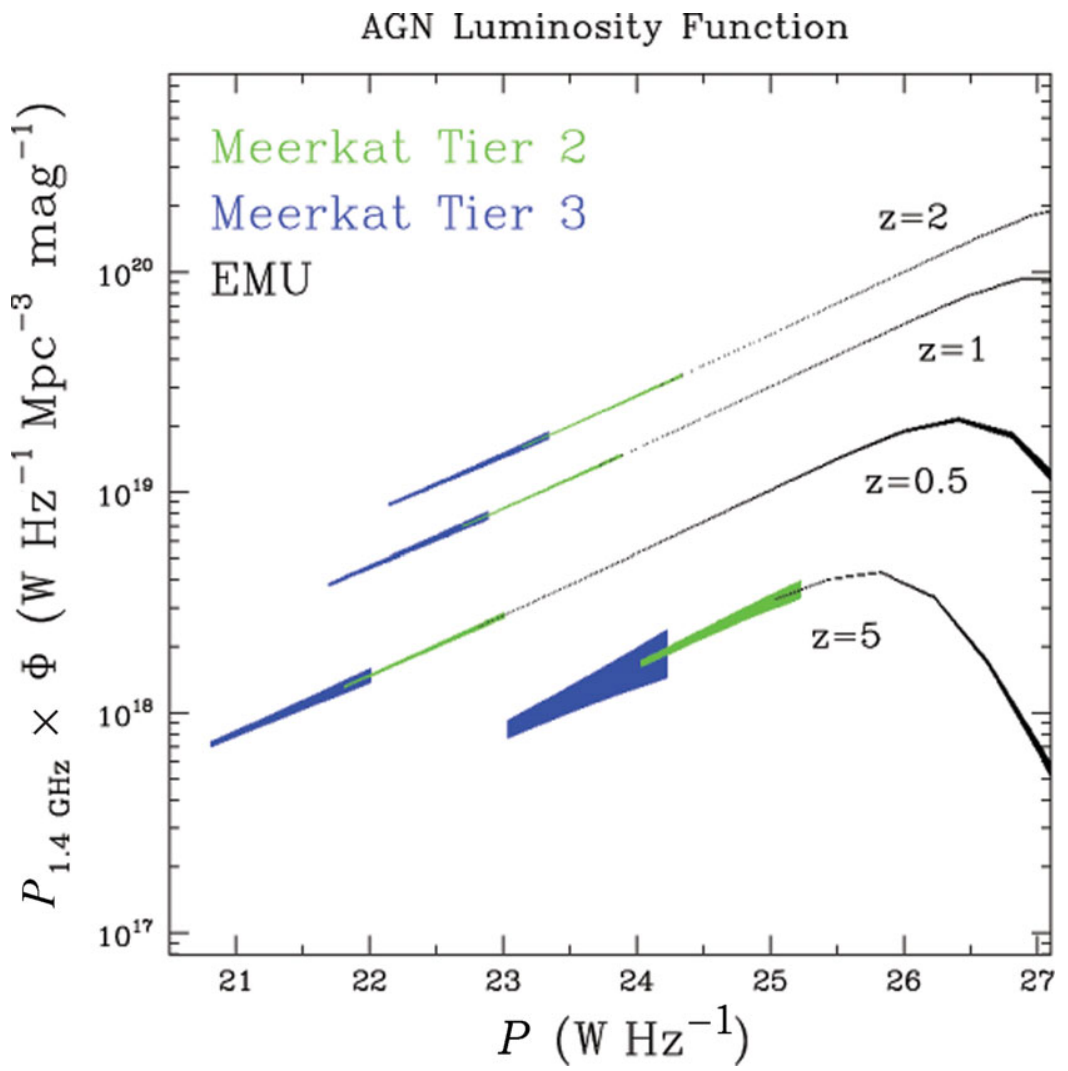

Figure 11. AGN luminosity function at different redshifts expected from the combination of ASKAP and MeerKAT deep surveys (based on models described by Prandoni, de Ruiter, \& Parma 2007).

jets (e.g. Merloni \& Heinz 2007; Hardcastle et al. 2007; Best et al. 2012). These jets pump energy into their environments, inflating cavities and bubbles in the surrounding intergalactic and intracluster medium (ICM), from which estimates of the mechanical energy associated with the jets can be made (e.g. Cavagnolo et al. 2010). Studies of radio AGNs in the nearby Universe have shown that the fraction of galaxies that host radio-loud AGNs (with $L_{1.4 \mathrm{GHz}}>10^{23} \mathrm{~W} \mathrm{~Hz}^{-1}$ ) is a strong function of stellar mass (e.g. Best et al. 2005), and that the time-averaged energetic output associated with recurrent radio source activity may indeed be sufficient to control the rate of growth of massive galaxies. However, sensitivity limits of current large-area radio surveys mean that these lowluminosity sources are only observed in the nearby Universe. How does the relation between galaxy mass and radio-AGN fraction (the radio source duty cycle) evolve with redshift out to the peak epoch of galaxy formation? Out to what redshift does radio-AGN heating continue to balance cooling? What is the differential evolution of the radiatively efficient and inefficient accretion modes (cf. Best et al. 2012), and is evidence for 'down-sizing' in the radio source population (e.g. Rigby et al. 2011) confirmed? What drives these processes? These are all questions for the next-generation radio arrays, coupled with high-quality multiwavelength data sets. Largearea surveys are required in order to study a sufficient volume to include the full range of galaxy environments, given the very important role that large-scale environment can play in the evolution of galaxies.

\subsubsection{Ultra-steep spectrum radio sources below the mJy barrier}

Another exciting prospect for the upcoming generation of radio continuum surveys is that of finding complete populations of high-redshift radio galaxies (HzRGs). These are among the most luminous galaxies and seem to be associated with the most massive systems (e.g. van Breugel et al. 1999; Jarvis et al. 2001a; Willott et al. 2003; Rocca-Volmerange et al. 2004; De Breuck et al. 2005; Seymour et al. 2007) often going through a phase of violent star formation at the $1000 \mathrm{M}_{\odot} \mathrm{yr}^{-1}$ level and showing large gas and dust reservoirs (e.g. Dunlop et al. 1994; Ivison 1995; Hughes, Dunlop, \& Rawlings 1997; Ivison et al. 1998; Papadopoulos et al. 2000; Archibald et al. 2001; Klamer et al. 2005; Reuland et al. 2003, 2004, 2007; but see Rawlings et al. 2004). HzRGs are considered to be the progenitors of the brightest cluster ellipticals and have been used as beacons to identify overdensities in the distant universe, i.e. proto-cluster environments at $z \sim 2.5$ (e.g. Stevens et al. 2003; Venemans et al. 2007). Identifying and tracing the evolution of HzRGs offers a unique path to study galaxy and large-scale structure formation 
and evolution from the earliest epochs, and extensive studies will finally become possible over the next few years.

Until recently, searches for HzRGs have been limited to wide-area (and thus relatively shallow) surveys. This is appropriate as the luminosities of HzRGs are large enough to make them detectable to very high redshifts (beyond $z \sim 5$ ) at flux densities of tens or even hundreds of mJy. For example, TN J0924-2201, the most distant known radio galaxy at $z=5.2$, exhibits a $1.4-\mathrm{GHz}$ flux of over $70 \mathrm{mJy}$ (De Breuck et al. 2000; van Breugel et al. 1999). But understanding a population, its origin and evolution, and how it fits in the overall galaxy population requires more than just the detection and study of the most extreme objects. The selection of more complete samples, over a wider redshift range, requires deeper surveys over wide areas. However, the large population of low $-z(z \lesssim 1-2)$ SF galaxies at sub-mJy levels presents a considerable difficulty for studies of HzRGs, as efficient ways of distinguishing between AGNs and SF galaxies are then necessary.

One of the most successful tracers of HzRGs relies on the relation between the radio spectral index and redshift (e.g. Tielens, Miley, \& Willis 1979; Chambers et al. 1996). Although an ultra-steep (radio) spectrum (USS; $\alpha \lesssim-1$ ) is not a necessary condition for a high redshifts, and, in fact, the USS selection misses a possibly large fraction of HzRGs (e.g. Waddington et al. 1999; Jarvis et al. 2001b, 2009; Schmidt, Connolly, \& Hopkins 2006), a higher fraction of high-redshift sources can be found among those with the steepest radio spectra. The USS criterion is so effective that most of the radio galaxies known at $z>3.5$ have been found using it (see Miley \& De Breuck 2008, for an overview of the selection of HzRGs). Surprisingly, there is still no satisfactory explanation for this spectral index-redshift correlation, as neither (a) a combination of an increased spectral curvature with redshift and the redshifting of a concave radio spectrum (e.g., Krolik $\&$ Chen 1991) to lower radio frequencies nor (b) radio jets expanding in dense environments, more frequently observed in distant proto-clusters, which would appear with steeper spectral indices (Klamer et al. 2006; Bryant et al. 2009; Bornancini et al. 2010) seem to explain the observations.

With the next generation of radio continuum surveys, the search for USS HzRGs will be extended to the whole sky and to microJansky flux levels. Although such collections have been assembled before (e.g. Bondi et al. 2007; Owen et al. 2009; Afonso et al. 2009; Ibar et al. 2009), only very recently has it been possible to characterise them. Using deep optical and infrared imaging of the Lockman Hole, Afonso et al. (2011) have started a detailed analysis of the $\mu \mathrm{Jy}$ USS radio source population, something that will be possible over a significant fraction of the sky using radio surveys such as EMU, WODAN, and LOFAR.

This first detailed analysis of the USS $\mu \mathrm{Jy}$ population used the deep multiwavelength coverage of the Lockman Hole. The sample selection was made using ultra deep VLA 1.4-GHz (reaching $6 \mu \mathrm{Jy} \mathrm{rms}$ ) and GMRT 610-MHz data (15 $\mu \mathrm{Jy} \mathrm{rms})$, and resulted in 58 sources with a spectral in-

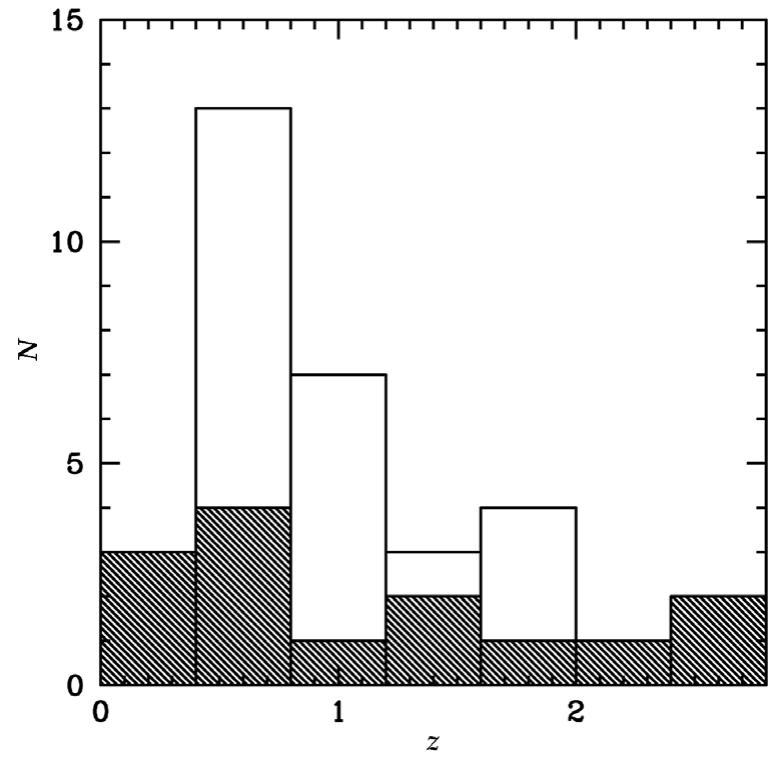

Figure 12. Redshift distribution for radio-faint USS sources in the Lockman Hole, from Afonso et al. (2011). Filled histogram denotes sources with a spectroscopic redshift determination, while the open region refers to photometric redshift estimates. A further 25 USS sources (43\% of the full sample) exist but with no redshift estimate, mostly at fainter $3.6 \mu$ m fluxes and likely to be found at higher redshifts.

dex of $\alpha^{1400}{ }_{610} \leq-1.3$. The deep IR coverage at $3.6 \mu \mathrm{m}$ and $4.5 \mu \mathrm{m}$ provided by the Spitzer Extragalactic Representative Volume Survey (SERVS; Mauduit et al. 2012) resulted in an extremely high identification rate, above the $80 \%$ level. Mid-infrared colours are compatible with a significant AGN presence among the radio-faint USS population. Spectroscopic redshifts for 14 sources and photometric redshifts for a further 19 sources were used for the redshift distribution of these sources, ranging from $z \sim 0.1$ to $\sim 2.8$ and peaking at $z \sim 0.6$ (see Figure 12). Twenty-five sources have no redshift estimate, including the faintest sources at infrared wavelengths, which indicates that higher redshifts are likely in this population. In this respect, they may be similar to the Infrared-Faint Radio Sources (IFRS), first identified by Norris et al. (2006), and which appear to be very high redshift dusty radio galaxies (Garn \& Alexander 2008; Huynh, Norris, \& Middelberg 2010; Middelberg et al. 2008a, 2011a; Norris et al. 2007, 2011a; Cameron et al. 2011; Zinn, Middelberg, \& Ibar 2011).

A comparison with the SKADS Simulated Skies models (Figure 13) indicates that FRIs and RQ AGNs may constitute the bulk of the USS population at $\mu$ Jy radio flux densities (FRIIs are thought to dominate above the mJy level). According to the models, the USS technique will be as efficient for the selection of very high- $z$ radio sources at $\mu \mathrm{Jy}$ fluxes as when applied at much higher flux density levels. This raises exciting prospects for the next generation of wide-field radio surveys, as it increases the potential for detailed studies of sources at very high redshifts and understanding of the evolution of the AGN activity in the Universe. 


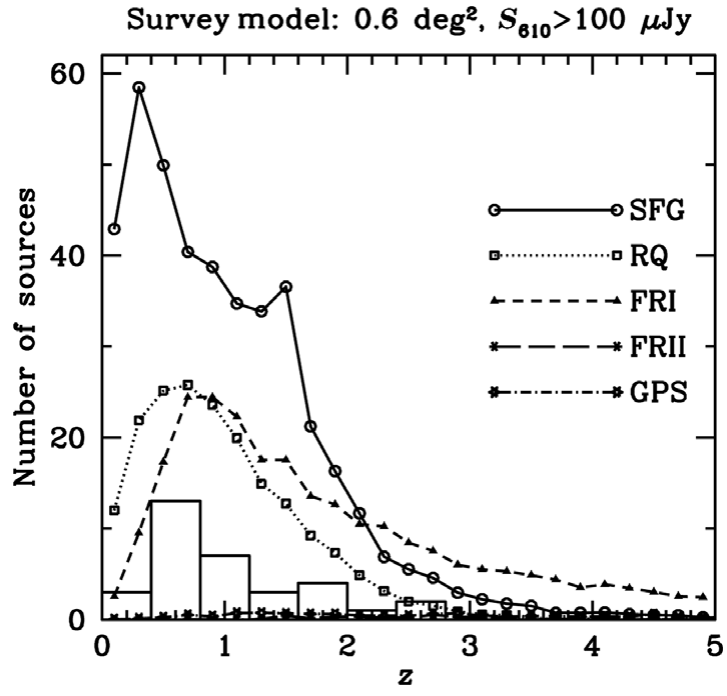

Figure 13. Predictions from the SKADS simulated skies models for the redshift distributions of radio source populations, irrespective of their radio spectral indices, for a radio survey reaching a detection sensitivity of $100 \mu \mathrm{Jy}$ at $610 \mathrm{MHz}$ over $0.6 \mathrm{deg}^{2}$, similar to the Lockman Hole radio survey considered in Afonso et al. (2011). The observed redshift distribution for USS sources in that work is also displayed.

These prospects are strengthened by the recent work of Ker et al. (2012), who have shown that redshift and spectral index are correlated in spectroscopically complete samples, and argue that this is due both to the dense intergalactic medium and the early evolutionary stage of the AGN, although they, together with Zinn et al. (2012), also show that the redshift-spectral index correlation is weaker than other potential redshift indicators.

\subsubsection{The luminosity-dependent evolution of the radio luminosity function}

Section 3.3.2 described how AGN radio jets interact with the surrounding intergalactic and ICM to prevent both large-scale cluster cooling flows and the continued growth of massive ellipticals (e.g. Fabian et al. 2006; Best et al. 2006, 2007; Croton et al. 2006; Bower et al. 2006). Determining the evolution of the RLF is therefore important for understanding the timescales on which they impose these effects.

A key early study of radio-loud AGN by Dunlop \& Peacock (1990) found increases of two to three orders of magnitude at a redshift of two, compared with the local Universe, in the comoving number density of both flat and steep spectrum radio-loud sources. They also saw indications of a highredshift $(z \sim 2.5)$ decline in density, but their work, and that of subsequent studies in this area (e.g. Shaver et al. 1996; Jarvis et al. 2001b; Waddington et al. 2001), has been limited by the inability to probe the depth and volume necessary to probe the high-redshift behaviour. This has motivated the development of the Combined EIS-NVSS Survey of Radio Sources (CENSORS; Best et al. 2003), which has been designed to maximise the information for high-redshift, steep spectrum sources close to the break in the RLF. CENSORS is a 1.4-
GHz-selected sample and contains 135 sources complete to a flux density of $7.2 \mathrm{mJy}$. It is currently $78 \%$ spectroscopically complete (Ker et al. 2012), with the remaining redshifts estimated via either the $K-z$ or $I-z$ magnitude-redshift relations.

The CENSORS sample, together with additional radio data, source counts, and local RLF, is used to investigate the evolution of the steep spectrum luminosity function via a new grid-based modelling method in which no assumptions are made about the high-redshift behaviour (Rigby et al. 2011). Instead, the RLF variations are determined by allowing the space densities at various points on a grid of radio luminosities and redshifts to each be free parameters, and then simply finding the best-fitting values to this many-dimensional problem. The modelling finds conclusive evidence, at $>3 \sigma$ significance, for a luminosity-dependent high-redshift decline in space density for the steep-spectrum sources. At low radio powers $\left(P_{1.4 \mathrm{GHz}}=10^{25}-10^{26} \mathrm{~W} \mathrm{~Hz}^{-1}\right)$ the space densities peak at $z \gtrsim 1$, but move to higher redshift for the higher powers $\left(z \gtrsim 3\right.$ for $\left.P_{1.4 \mathrm{GHz}}>10^{27} \mathrm{~W} \mathrm{~Hz}^{-1}\right)$. This is illustrated in Figure 14, and is similar to the 'cosmic downsizing' seen for other AGN populations (e.g. De Zotti et al. 2010; Wall 2008; Hasinger, Miyaji, \& Schmidt 2005; Richards et al. 2005). These results are robust to the estimated redshift errors and to variation in the radio spectral index with redshift.

The evolution of low-luminosity radio AGNs is less well understood than that of radio-loud AGNs, with some studies finding no evidence for any evolution of the RLF for low-luminosity radio AGNs (e.g. Clewley \& Jarvis 2004) and others finding that low-luminosity AGNs (LLAGNs) do evolve with redshift, although more slowly (less than a factor of 10 from $z=0$ to 1.2) than their high-luminosity counterparts (Smolčić et al. 2009; McAlpine \& Jarvis 2011). Best et al. (2012) suggest that the luminosity dependence of the evolution of the AGN RLF may be attributed to the varying fractions of hot and cold mode sources with redshift. Recent results (Mauch \& Sadler 2007; Sadler et al. 2007; Padovani et al. 2011; Mao et al. 2012) fail to resolve this discrepancy, with ambiguity added by factors such as evolution, cosmic variance, differences in classification, differences in terminology, and the difficulty of distinguishing LLAGNs from SF galaxies, or the bulge from the disc of a galaxy. Nextgeneration radio surveys using SKA pathfinders can resolve this discrepancy provided we develop reliable techniques for distinguishing the AGN component from the SF component of a galaxy.

\subsubsection{Environment}

Studies of galaxies in groups and clusters by Best et al. (2007) and Wake et al. (2008) have shown that the environment of a radio galaxy has a significant effect on the radio emission. Sabater et al. $(2008,2012)$ built on this result by comparing (a) the radio AGN activity in a sample of isolated galaxies (AMIGA sample; Verdes-Montenegro et al. 2005) with that of a matched sample of galaxies in clusters (Miller \& Owen 2001; Reddy \& Yun 2004) and (b) the optical AGN activity for the AMIGA sample with that of a matched sample of 


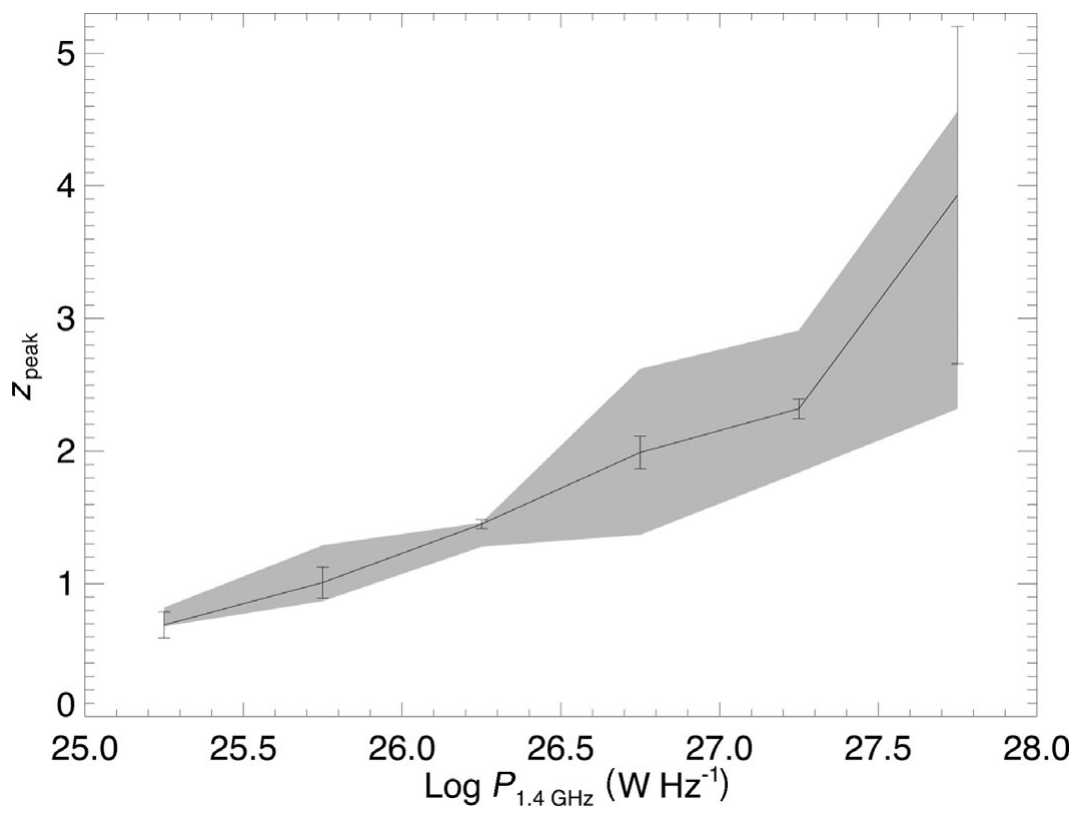

Figure 14. The dependence of the redshift of the peak in space density $\left(z_{\text {peak }}\right)$ on radio power for the best-fitting steep spectrum grid, showing that the numbers of the most powerful radio sources peak at the highest redshifts (Rigby et al. 2011). The shaded region and error bars give two different determinations of the spread in the measurements.

galaxies in compact groups (Martinez et al. 2010). The results of all these studies show that radio AGN activity is strongly influenced by the environment while this influence is less clear for optical AGN activity (Miller et al. 2003).

\subsubsection{Black hole spin}

A further area of study from radio observations is the study of black hole physics. For a given accretion rate, as traced by the X-ray to mid-infrared SED, AGNs show a huge range of radio luminosities. This suggests that some extra parameter, independent of the accretion rate, is controlling the jet power of the AGN. The spin of the black hole is a very attractive candidate, for both observational and theoretical reasons (e.g. Wilson \& Colbert 1995). Spin is effectively a hidden parameter, which will barely show itself in the SED of the AGN. In addition, spinning black holes can convert rotational energy into jet power, meaning that jets can be powered with nominal efficiencies close to or exceeding unity. Observations of the most powerful radio AGNs suggests the jet power is comparable or larger than the rate of accretion of energy (e.g. Fernandes et al. 2011).

Radio observations allow us to estimate the jet power, and combining these with observations at X-ray energies or optical wavelengths can allow an estimate of the accretion rate and spin. For example, Martínez-Sansigre \& Rawlings (2011) consider the jet powers of SMBHs with $m_{\bullet} \geq 10^{8} \mathrm{M}_{\odot}$. The authors use a suite of semi-analytic models and general relativistic (GR) magnetohydrodynamic (MHD) simulations that predict the jet efficiencies as a function of spin, and find that these efficiencies can approximately explain the radio loudness of optically bright quasars.
Martínez-Sansigre \& Rawlings (2011) also find that they can explain the local RLF of high- and low-excitation galaxies independently (HEGs and LEGs, respectively), and that the HEGs and LEGs have different best-fitting spin distributions. The LEGs, modelled as advection-dominated accretion flows (ADAFs) or low-accretion rate SMBHs, have a bimodal spin distribution, with the SMBHs having typically low $(\hat{a} \sim 0)$ or high $(\hat{a} \sim 1)$ spins. The probability density of the high-spin peak is significant, typically $\sim 33 \%$ of the total probability density. On the other hand, the HEGs, modelled as SMBHs accreting at a high fraction of their Eddington rate, are found to have typically low spins ( $\hat{a} \sim 0$ only), with a small or non-existent component of high spin. The radio LFs of HEGs and LEGs given by these spin distributions can be extrapolated to high redshift, where they correctly reproduce the total radio LF of all AGNs at $z=1$. Due to the strong evolution of the high-Eddington rate SMBHs (as reflected by the evolution of the X-ray LF), this modelling also predicts that the $z=1$ radio sources above a radio luminosity density $\sim 10^{26} \mathrm{~W} \mathrm{~Hz}^{-1} \mathrm{sr}^{-1}$ (at $151 \mathrm{MHz}$ ) should show exclusively HEG spectra, while the fraction of LEGs should increase rapidly at lower radio luminosity densities.

Since the space density of HEGs increases rapidly, the typical spin of SMBHs will change with redshift. At $z \sim 0$, most of the SMBHs will have the bimodal distribution inferred for the LEGs, with a high fraction of SMBHs having a high spin (Figure 15, top). At $z \gtrsim 1$, the space density of HEG radio sources is larger than that of LEG sources, so that the SMBHs will typically have the distribution of spins of the HEGs, dominated by a low-spin component (Figure 15, bottom). 


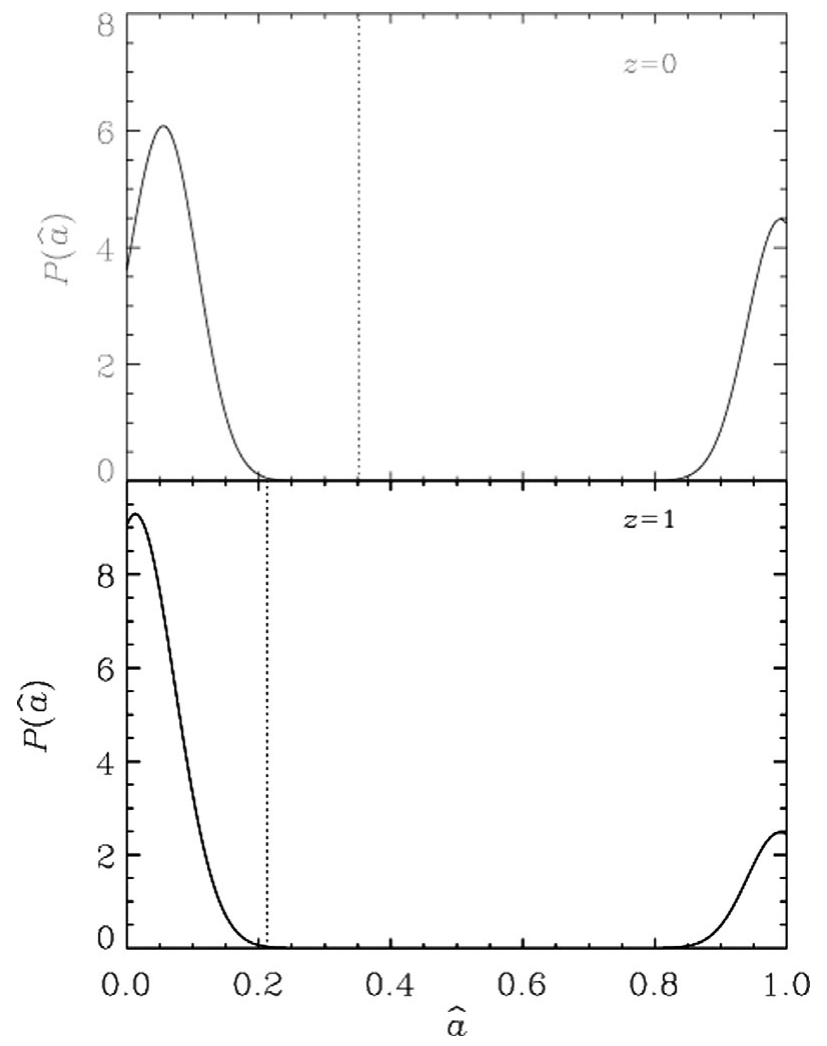

Figure 15. Inferred spin distributions for SMBHs at $z=0$ and 1, from Martínez-Sansigre \& Rawlings (2011). The vertical dashed lines mark the mean value at each redshift. Bottom: the spin distribution at $z=1$ is dominated by the low-spin population, with a minor component of high-spin SMBHs. Top: at $z=0$ a larger fraction of SMBHs possess a high spin, so that the mean spin is higher.

The evolution of the spin from SMBHs (with $m_{\bullet} \geq 10^{8}$ $\mathrm{M}_{\odot}$ ) can hence be described as evolving from predominantly low spins in high-Eddington rate systems at $z \gtrsim 1$, to a bimodal spin distribution at $z \sim 0$, for mostly low-Eddington rate systems. At the epoch of highest accretion, the typical spin is low and at later epochs the fraction of SMBHs with high spins increases (Figure 16). This can be understood in terms of chaotic accretion (e.g. King, Pringle, \& Hofmann 2008) spinning the accreting SMBHs down, whereas major mergers spin the SMBHs up (e.g. Rezzolla et al. 2008). At earlier epochs, there is a plentiful supply of cold gas, so that accretion spins SMBHs down. As this gas disappears, however, the breaking mechanism that spins SMBHs down is removed, while some SMBHs will still experience major mergers. Hence, a population of high-spin SMBHs gradually appears.

\subsection{Galaxy clusters and large-scale structure}

Clusters of galaxies are the most massive systems in the present Universe. They are not isolated regions, but evolving regions at the intersections of filaments and sheets in the large-scale structure.
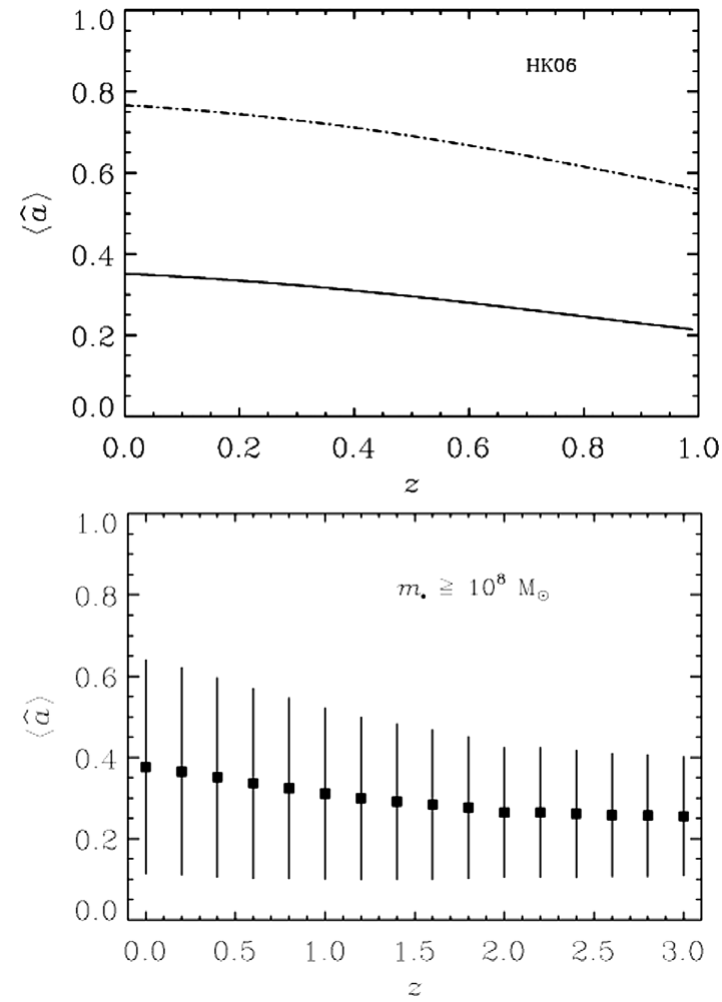

Figure 16. Top: mean spin of SMBHs with $m_{\bullet} \geq 10^{8} \mathrm{M}_{\odot}$, from MartínezSansigre \& Rawlings (2011). There is a gradual increase of the mean spin between $z \gtrsim 1$ and $z=0$, which is most likely due to a fraction of SMBHs undergoing major mergers and subsequent chaotic accretion. Bottom: cosmological simulation of the mean spin from Fanidakis et al. (2011), which includes chaotic accretion and mergers, and which is in excellent agreement with the inference based on radio observations (top).

Tens of thousands of clusters are currently known, but only a few are known at $z>1$ (Wilson et al. 2008; Kodama et al. 2007), with the highest cluster redshift at $z=2.07$ (Gobat et al. 2011).

Radio observations of galaxy clusters are powerful probes of the physics of the ICM, but they also provide a complementary way to trace the evolution of large-scale structure of the Universe up to very large distances. These studies also have the potential to explore the way in which galaxy evolution (star formation and nuclear activity) depends on its environment.

Radio observations have shown the existence of diffuse large-scale synchrotron radiation from the ICM, implying that non-thermal components, magnetic fields and relativistic particles, are mixed with the hot ICM. The origin of these components is still unclear and a subject of lively debate. Potentially these components contribute to the energy of the ICM and drive complex physical processes that may significantly alter our present (simplified) view of the ICM (Schekochihin et al. 2005; Subramanian, Shukurov, \& Haugen 2006; Brunetti \& Lazarian 2011). 
Radio emission from clusters of galaxies is generated both by the constituent galaxies and by diffuse emission in the ICM.

The radio emission from constituent galaxies often extends well beyond the galaxy optical boundaries and interacts with the ICM. This interaction is observed in tailed radio galaxies (see Section 3.4.3), and radio sources filling X-ray cavities at the centre of cool-core clusters (McNamara \& Nulsen 2007; Feretti \& Giovannini 2008).

Diffuse sources are typically grouped into three classes (Feretti \& Giovannini 1996; Kempner et al. 2004):

- Haloes, which are steep-spectrum Mpc-scale diffuse sources in the central regions of X-ray luminous clusters (e.g. Ferrari et al. 2008; Venturi 2011; Feretti et al. 2012),

- Relics, which are located in the peripheral regions of both merging and relaxed clusters and caused by cluster-cluster shocks,

- Mini-haloes, which are hosted in relaxed cool-core clusters, are centrally located, and usually surround a powerful radio galaxy, such as 3C84 in the Perseus cluster.

Even amongst these classifications, there is ambiguity, with the term 'relic' being used to describe both old galaxies and shock-accelerated regions of diffuse electrons. Furthermore, some amorphous radio objects, such as the diffuse 'Phoenixes' (Kempner et al. 2004) and 'roundish relics' (Feretti et al. 2012), cannot easily be so classified.

There are two ways in which these classifications could be improved. First, we could build in more physics to link observations to thermal and non-thermal physical processes in galaxy clusters, but must ensure that current observational/theoretical expectations do not bias our classification. Second, we could use a comparison of radio and X-ray surface brightness distributions.

SKA pathfinder surveys will discover hundreds of diffuse radio haloes in regions of the sky where there are no known clusters (Cassano et al. 2012) and even more tailed radio galaxies (Mao et al. 2010a; Norris 2011). Radio emission in clusters is therefore likely to become an important tool both for studying clusters themselves, and for detecting large numbers of clusters to study cosmology and trace large-scale structure formation.

\subsubsection{Radio haloes}

About 40 giant radio haloes are known so far in the redshift range $0 \leq z \leq 0.55$. The GMRT Radio Halo Survey (Venturi et al. 2008), in combination with the NVSS and WENSS surveys, allows a statistical exploration of radio haloes in galaxy clusters in the redshift range $0 \leq z \leq 0.4$ (Cassano et al. 2008). The cluster radio properties were found to be bimodal: only a fraction of massive clusters host haloes, while no $\mathrm{Mpc}$-scale radio emission is detected in the majority of clusters at current sensitivity levels. The radio luminosity of these 'radio-quiet' clusters is less than one-tenth of that of the observed radio haloes (Brunetti et al. 2007, 2009). Xray follow-up of these observations shows that radio haloes are found only in merging clusters while 'radio-quiet' clusters are systematically more relaxed systems (Cassano et al. 2010a).

The radio properties of haloes correlate with the thermal properties of the parent clusters and the radio power of haloes increases with X-ray luminosity, suggesting a direct connection between the thermal and non-thermal cluster properties (Feretti et al. 1999, 2012; Bacchi et al. 2003; Cassano et al. 2006; Brunetti et al. 2007, 2009).

According to a prominent scenario for the origin of giant radio haloes, a fraction of the gravitational binding energy of dark matter haloes released during cluster mergers can be channelled into turbulence. This turbulence may in turn accelerate relativistic particles (Brunetti et al. 2001; Petrosian 2001) explaining the connection observed between radio haloes and cluster mergers.

The source of the synchrotron electrons in these haloes is unclear, as acceleration of electrons from the thermal pool to relativistic energies by MHD turbulence in the ICM cannot provide sufficient energy (Petrosian 2008). Instead, there must be a pre-existing population of relativistic (or suprathermal) seed particles that are then reaccelerated by turbulent acceleration during mergers. One possibility is that the seeds are secondary electrons generated by collisions between CRs and thermal protons (Brunetti \& Blasi 2005; Brunetti \& Lazarian 2011). These hybrid models predict that Mpc-scale synchrotron emission from secondary electrons ('pure hadronic haloes') must also be present in relaxed clusters, with a radio luminosity 10-20 times smaller than that of merging clusters (Brunetti \& Lazarian 2011). This emission should be detectable with EMU/WODAN. Figure 17 shows the 1.4-GHz RLF of radio haloes in the local Universe $(z=0.1)$ : at higher radio luminosities, turbulent cluster haloes dominate, but at lower luminosities the RLF is dominated by 'hadronic' haloes in relaxed systems. Additional sources of seed supra-thermal electrons include shocks, AGNs, galaxies, and magnetic reconnection, so relaxed clusters may have an even higher radio luminosity. If EMU fails to detect radio haloes in relaxed clusters, this will provide a strong constraint on secondary particles and on the energy content of CRs in the ICM.

An extrapolation of the radio power of haloes at $1.4 \mathrm{GHz}$ versus the cluster X-ray luminosity indicates that clusters with $L_{x}<10^{44} \mathrm{erg} \mathrm{s}^{-1}$ should host haloes of power $L_{1.4}$ $<10^{23} \mathrm{~W} \mathrm{~Hz}^{-1}$. With a typical size of $1 \mathrm{Mpc}$, their surface brightness could be too low to be detected at $1.4 \mathrm{GHz}$. However, less powerful radio haloes should be also smaller in size (Feretti et al. 2012), implying that they should be easily detected by the EMU survey. A comparison between 1.4 GHz data with low-frequency data (LOFAR) will be crucial to test turbulence properties. A large number of 'hadronic haloes' may be detected at $1.4 \mathrm{GHz}$ (see Figure 17), emphasising the importance of comparing the EMU and LOFAR surveys. 


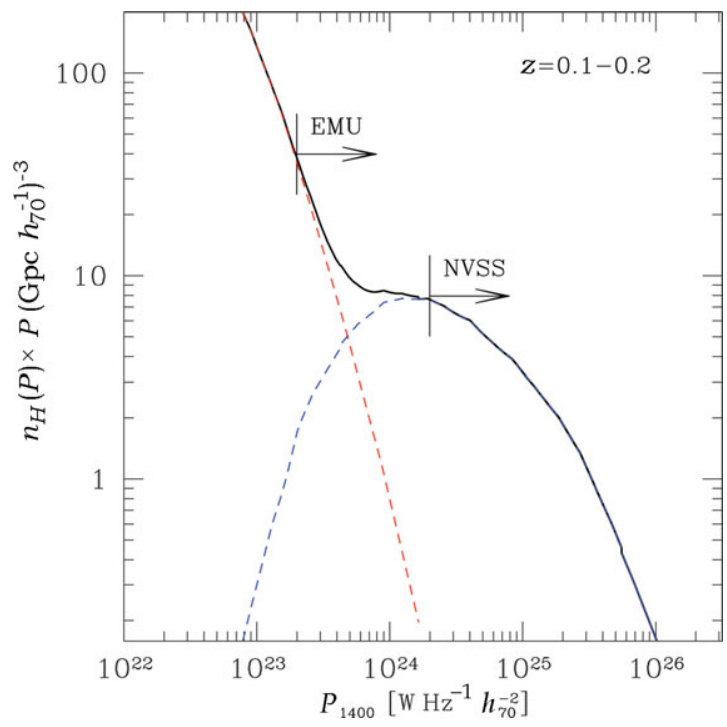

Figure 17. Luminosity functions at $1.4 \mathrm{GHz}$ of giant radio haloes in the redshift range $0.1 \leq z \leq 0.2$. The red dashed line marks the contribution from 'hadronic' haloes in relaxed clusters, while the blue dashed line marks the contribution from haloes in merging 'turbulent') clusters. The black solid line gives the total luminosity functions (Cassano et al. 2012). The arrows show the EMU and NVSS sensitivities at that redshift.

If turbulence is responsible for the origin of haloes, then the radio-emitting electrons are accelerated up to energies $m_{e} c^{2} \gamma_{m} \leq$ several $\mathrm{GeV}$, where the synchrotron and IC losses quench the acceleration by MHD turbulence. Radio haloes will therefore steepen at a frequency $v_{s} \propto \gamma^{2}{ }_{m} b$, making it difficult to detect haloes at higher frequencies (Brunetti et al. 2008).

Only the few most energetic mergers in the Universe can generate giant radio haloes with $v_{s} \geq 1 \mathrm{GHz}$ (Cassano et al. 2005). Most mergers instead consist of major mergers between less massive systems, or minor mergers in massive systems (Cassano et al. 2006, 2010b), which will therefore have steeper spectra ( $\operatorname{smaller} v_{s}$ ). Recent Monte Carlo calculations based on turbulent acceleration in galaxy clusters predict that LOFAR, reaching rms $=0.1 \mathrm{mJy}^{\text {beam }}{ }^{-1}$ at $120 \mathrm{MHz}$ over the northern hemisphere, will detect more than 350 radio haloes at redshift $z \leq 0.6$, increasing the number of known haloes by a factor of nearly ten (Enßlin \& Röttgering 2002; Cassano et al. 2006). More than half of these haloes will be 'ultra-steep spectrum' haloes, with $v_{s}<600 \mathrm{MHz}$ (Cassano et al. 2010b), providing a clear test of the importance of turbulence in particle acceleration in merging clusters.

\subsubsection{Radio relics}

Relics (occasionally termed radio phoenixes) are irregular, elongated diffuse sources which are generally located at the periphery of the cluster, with the linear size of $400-1500 \mathrm{kpc}$ (Giovannini \& Feretti 2002). Most have a steep spectrum $(\alpha<$ -1 ), and are linearly polarised at a level of $\sim 10 \%-30 \%$. They show an asymmetric transversal profile and spectral index distribution. Current models suggest that they are caused by diffusive shock acceleration of electrons in old radio-emitting regions, triggered by a cluster merger event. Currently, 34 clusters are known to contain at least one relic source. Eleven of these also have a radio halo and ten have a double relic.

The existence of relic sources suggest cluster-wide magnetic fields of about $0.1-1 \mu \mathrm{G}$ and relativistic electrons of $\sim$ $\mathrm{GeV}$ energies in large peripheral regions where the density of the hot ICM is low and galaxies are scarce.

Few radio relics have been well studied and few have deep high resolution radio maps, so that their size and flux density may be underestimated. Most radio spectra have been obtained with a limited frequency range and very few spectral index images have been published. Because most relics are located at the cluster periphery, they are often outside, or at the edge, of available X-ray images, making it difficult to compare radio and X-ray data. However, a few relics have been found and studied in detail (e.g. Johnston-Hollitt 2003; van Weeren et al. 2010; Brown, Duesterhoeft, \& Rudnick 2011).

A few relics show a curved shape radio spectrum and a diffuse, filamentary morphology. They often have been found near the central $\mathrm{cD}$ galaxy, suggesting a possible connection with a past activity of this galaxy, but in A1664 (see Figure 18) and in A548b (Feretti 2006) the relic is far from the cluster centre.

Figure 19 compares the radio and X-ray power of radio haloes and relics, showing that both the relic and halo power are correlated with the parent cluster X-ray luminosity, although this may be influenced by selection effects. The relics have a larger dispersion than the haloes, confirming that shocks are more efficient than turbulence in accelerating relativistic electrons or amplifying magnetic fields. This correlation confirms the link between relics and cluster mergers and shows that the SPARCS surveys will enable the study of large-scale magnetic fields in low density regions at the cluster periphery. Such correlations have recently been discussed by Feretti et al. (2012).

\subsubsection{Tailed radio galaxies}

Tailed radio galaxies include wide-angle tails (WATs) and narrow-angle tails (NATs). They are common in large clusters (Blanton et al. 2000, 2001, 2003) and appear to represent radio-loud AGNs in which the jets are distorted by the ICM (Mao et al. 2010a). Mao et al. (2011b) have found six tailed galaxies in the $7 \mathrm{deg}^{2}$ of the Australia Telescope Large Area Survey (ATLAS), suggesting that $\sim 40000$ new clusters will be detected by EMU, using the tailed galaxies as tracers. With higher spatial resolution, more tailed galaxies become visible, and Dehghan et al. (2011), using high-resolution images of the same ATLAS fields, find 12 tailed galaxies in $4 \mathrm{deg}^{2}$, implying $\sim 10^{5}$ tailed galaxies in EMU. Importantly, such galaxies can be detected out to high redshifts of $z \sim$ 0.5 and beyond (Wing \& Blanton 2011; Mao et al. 2010a), providing a powerful diagnostic for finding clusters. 


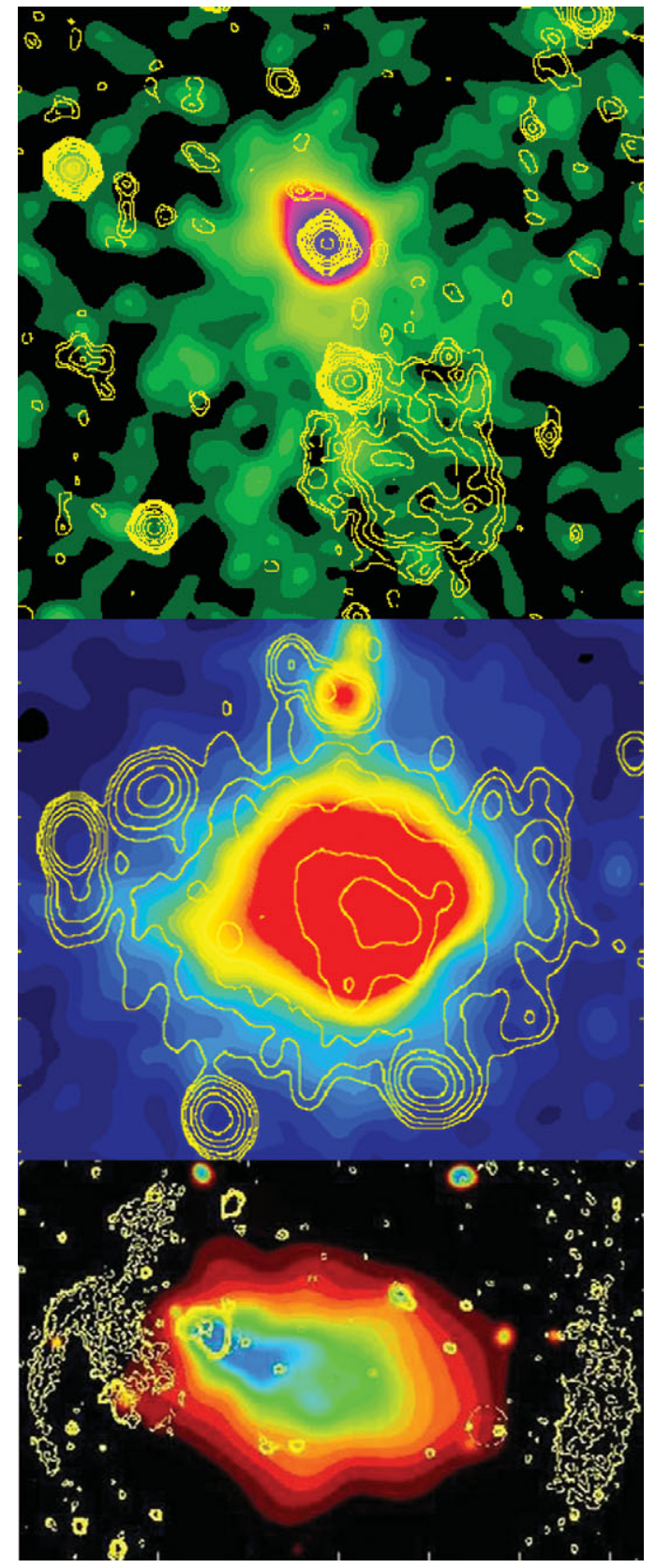

Figure 18. Radio image (contours) overlaid on X-ray images (colour) of three representative clusters: (top) the radio relic in the cluster A1664 (Govoni et al. 2001), (centre) the giant radio halo in A2163 (Feretti et al. 2001), and (bottom) the double relic in A3376 (Bagchi et al. 2006).

\subsection{The magnetic sky}

Magnetic fields are very important at all scales in the Universe, exerting their influence at very small scales in the ISM, at large scales in galaxies and clusters, and on the vast scale of cosmic filaments. Yet we still have limited understanding of the magnetic contribution to the energy balance in these structures, and their dynamical influence and contribution to the evolution of different classes of object. Our best tools for measuring cosmic magnetic fields are radio observations of

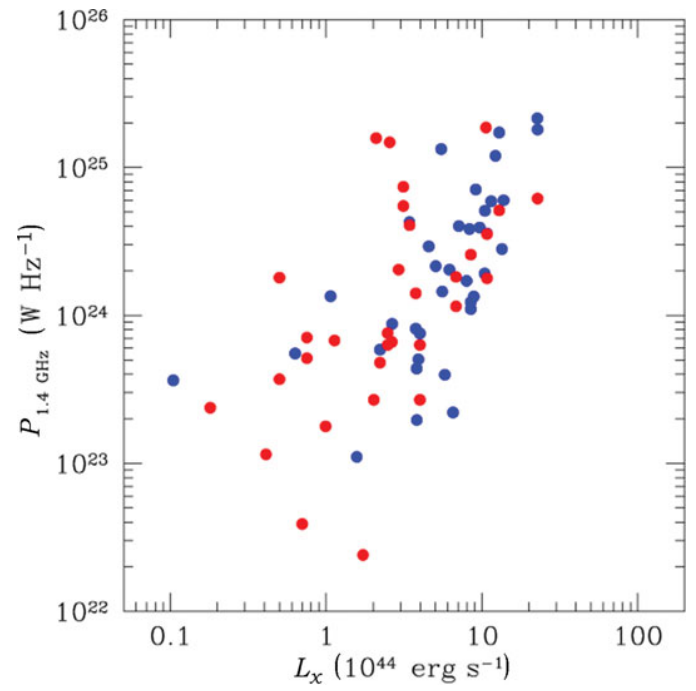

Figure 19. Halo (blue) and relic (red) radio power at $1.4 \mathrm{GHz}$ in units of $\mathrm{W} \mathrm{Hz} \mathrm{z}^{-1}$ versus cluster X-ray luminosity.

synchrotron radiation and its linear polarisation, which are sensitive to total and ordered magnetic fields, respectively, and Faraday rotation, which is sensitive to magnetic fields along the line of sight.

One of the areas which will benefit most from SKA pathfinder surveys is the study of radio continuum polarisation. The prospects for wide-field deep imaging, with broad wavelength coverage, and (in PAF-equipped telescopes) with excellent control over the primary beam response, combine to make the suite of pathfinders excellent for the study of polarisation. The two major polarisation surveys which are currently planned with SKA pathfinders are (i) the LOFAR Key Science Project on Cosmic Magnetism (see Section 2.8) and (ii) ASKAP-POSSUM project (see Section 2.4). They are highly complementary, spanning more than a decade in observing frequency, and will probe widely differing regimes of magnetised plasmas. Moreover, together they can observe both hemispheres. There are a number of other surveys aimed at recovering high density grids of rotation measures (RM grids) towards different astrophysical regions, notably the APERTIF-BEOWULF and APERTIF-FRIGG surveys (Section 2.2), the $e$-MERLIN Super-CLASS survey (Section 2.5; Battye et al. 2011), and the RM grid component of the MeerKAT MIGHTEE Survey (Section 2.9).

The primary tool for analysing the polarisation data from the pathfinders is the 'RM synthesis' technique (Burn 1966; Brentjens \& de Bruyn 2005). The essence of this technique is to perform a Fourier transform of the observed complex polarisation vector from the frequency (strictly, $\lambda^{2}$ ) domain to the 'Faraday depth' domain. The Faraday depth refers to the line-of-sight integral of the thermal electron density and magnetic field strength. In simple situations, it is more commonly known as the Faraday RM, while this simple relation breaks down for synchrotron emission and Faraday rotation in the same medium, various emitting and rotating layers 
along the line of sight, or more than one polarised feature with different RMs within an angular resolution element. The output of the RM synthesis technique is a reconstruction of the Faraday dispersion function, which describes the complex polarisation vector as a function of the Faraday depth (Brentjens \& de Bruyn 2005; Heald 2009). Other techniques for identifying magnetic field structures from RM synthesis observations continue to be developed (e.g. Beck et al. 2012).

The technique allows the detection of very faint polarised emission even when the RM is very high (i.e. corresponding to the polarisation angle rotating by more than a turn across the observing band) and avoids $n \pi$ ambiguities. Better precision in the Faraday depth domain is obtained by observing with wide bandwidth, and access to high values of $|R M|$ is provided by high spectral resolution-both of which are included in the specifications of all of the pathfinders.

High-quality polarisation observations address diverse science goals, ranging from local to cosmological scales. Here, we summarise a subset of these and discuss the impact of the pathfinder surveys on these fields.

\subsubsection{The large-scale structure of the Milky Way magnetic field}

Knowledge of the strength and orientation of the large-scale magnetic field in the Milky Way is crucial for studies of the origin and evolution of Galactic magnetism, but is also valuable for extragalactic topics such as ultra-high energy CRs, CMB polarisation, and magnetism in external galaxies, galaxy clusters, and intergalactic space.

Models constrained by observational RM data from pulsars, extragalactic sources, and diffuse Galactic synchrotron emission attempt to determine reversals in the spiral magnetic pattern of the Milky Way, and to measure the large-scale configurations of the field in the Milky Way disc and halo (e.g. Beck 2011; Noutsos et al. 2008; Van Eck et al. 2011; Sun et al. 2008; Jansson et al. 2009; Nota \& Katgert 2010; Mao et al. 2010b), all of which are key to understanding the evolution of galactic magnetic fields. However, controversy still exists about the existence and location of reversals, and the overall 3D configuration of the magnetic field in the Milky Way's disc and halo.

Major constraints to these large-scale magnetic field models will come from the rotation measure grid of RMs from compact extragalactic sources, most of which probe Galactic Faraday rotation. The SKA pathfinders ASKAP, LOFAR, APERTIF, and MeerKAT have complementary programs to observe RMs of extragalactic point sources to construct an RM grid with an average density of 100 RMs per square degree over the entire sky. These efforts will provide a polarised source density two orders of magnitude higher than the recent NVSS RM catalogue (Taylor, Stil, \& Sunstrum 2009), which has already proved useful for a wide range of analyses (e.g. McClure-Griffiths et al. 2010; Schnitzeler 2010; Stil, Taylor, \& Sunstrum 2011).

\subsubsection{The turbulent, magnetised interstellar medium}

Magnetised turbulence in the ISM plays a major role in many physical processes such as star formation, total ISM pressure equilibrium, CR scattering, gas mixing, and radio wave propagation (Scalo \& Elmegreen 2004).

At high Galactic latitudes, in the Galactic thick disc and halo, RM variations are small, and can be observed at long wavelengths. Spectropolarimetric observations of the Galactic foreground emission at mid-latitudes around $350 \mathrm{MHz}$, made using WSRT, show a wealth of small-scale polarisation at angular resolutions down to a few arcmin (Haverkorn, Katgert, \& de Bruyn 2003a, 2003b; Schnitzeler et al. 2007; Schnitzeler, Katgert, \& de Bruyn 2009). At even lower frequencies of $150 \mathrm{MHz}$, Bernardi et al. (2009) observed abundant structure in diffuse polarisation at intermediate latitudes. Therefore, LOFAR RM synthesis observations at mid- and high-latitude are expected to discover a wealth of small-scale RM structures in the magneto-ionised gas. This will be used to characterise the power spectrum of magnetic field fluctuations at high latitudes, i.e. in the Galactic gaseous halo and disc-halo connection.

In addition, calculation of the spatial gradient of diffuse polarisation can allow direct visualisation of turbulent structures in the ISM, from which we can infer fundamental properties such as Mach number and Alfvén speed (Gaensler et al. 2011; Burkhart, Lazarian, \& Gaensler 2012).

Closer to the Galactic plane, the larger RM values will cause partial or complete depolarisation of low-frequency emission, but are well suited to observations with ASKAP, APERTIF, and MeerKAT. The RM grid allows statistical analysis of interstellar magnetised turbulence as a function of position (Haverkorn et al. 2008), while RM synthesis of the diffuse polarised emission allows disentangling of synchrotron emitting and Faraday rotating layers (Brentjens 2011). It will also be possible to map out magnetic field strength and structure in discrete structures such as supernova remnants, planetary nebulae, superbubbles, $\mathrm{H}$ II regions, Galactic chimneys, and high-velocity clouds (see, e.g., Beck \& Gaensler 2004).

\subsubsection{Faraday screens}

At $1.4 \mathrm{GHz}$, the diffuse polarised sky typically bears little resemblance to the total intensity sky (e.g. Landecker et al. 2010). A widely accepted interpretation for this characteristic is that the detected polarised features are the signature of Faraday rotation rather than structure in the Galactic synchrotron emission. The exceptions are supernova remnants (Section 3.8.3) and pulsar wind nebulae (Section 3.8.4).

Observations have shown that the synchrotron emission of external galaxies is very smooth and the WMAP results have confirmed that for our Galaxy too. However, at lower radio frequencies, surveys show a lot of small-scale structure caused by Faraday rotation. A typical radio telescope operating in the 1.4-GHz wavelength range proves to be more 
sensitive to the Faraday rotation of an ionised and magnetised medium than to its bremsstrahlung signature.

The Faraday rotation structures we find in linear polarisation images are called 'Faraday Screens'. These Faraday Screens are still not well understood. Questions about these features we have to answer include:

- Are those features created by variation of the magnetic field or the free electron distribution (or a combination of both)?

- Can we correlate (or anti-correlate) these features with other tracers of the ISM?

- How can we explain features that do not correlate with anything?

- Can RM synthesis help to reveal the characteristics of these features?

- Can we relate these features with structures seen in the $\mathrm{RM}$ distribution determined from extra-galactic point sources?

A large wide-field survey such as POSSUM will help to better understand these features and reveal their true nature

\subsubsection{Nearby galaxies}

Spiral galaxies have typical magnetic field strengths of the order of $10 \mu \mathrm{G}$. They typically show ordered field patterns consistent with the spiral pattern in the plane, a higher level of magnetic turbulence in the arms themselves, and an Xshaped configuration in the high-latitude regions. A concise review of the properties of magnetic fields in galaxies is provided by Beck (2009).

The WSRT-SINGS Survey has investigated the global magnetic field properties across a small sample of nearby galaxies with diffuse polarised emission (Braun, Heald, \& Beck 2010). This work showed that azimuthal patterns in polarised intensity and RM (Heald, Braun, \& Edmonds 2009) are consistent with a magnetic field configuration consisting of an axisymmetric spiral in the disc and a quadrupolar poloidal pattern away from the plane, as predicted by the dynamo theory of magnetic field amplification (e.g. Widrow 2002). A critical component of the model is that turbulent magnetic fields in the SF disc depolarise synchrotron radiation from the far side of the disc (see Burn 1966, for a description of this Faraday dispersion effect). In face-on galaxies this effect, together with trailing spiral arms, results in the observed patterns. At higher and lower radio frequencies, the effects of Faraday dispersion will be reduced and enhanced, respectively, giving rise to different patterns in the polarised synchrotron radiation.

The polarisation surveys planned with ASKAP and LOFAR will thus be complementary in that they will trace ordered magnetic fields in significantly different volumes within nearby galaxies. Although depolarisation by Faraday dispersion is much stronger at LOFAR frequencies, this is mitigated by the fact that CR electrons that have diffused far from their acceleration sites (i.e. regions of massive star formation) will have lower energies because of synchrotron losses. Thus, synchrotron radiation from this aged CR population will peak at LOFAR frequencies, and should trace ordered fields in the far outer regions of galaxies. At the other end of the spectrum, high-frequency observations with MeerKAT and the VLA can 'see through' the SF disc, and trace the fields in the SF regions directly. The suite of SKA pathfinders can thereby provide complementary polarimetric studies leading to an 'onion-peel' polarimetric view of diffuse synchrotron emission in nearby galaxies.

\subsubsection{Magnetic field evolution in galaxies}

Beyond the nearby Universe, SKA pathfinder telescopes have insufficient angular resolution to map the synchrotron radiation across discs of galaxies. However, Stil et al. (2009) have shown that polarised radiation with a preferred, and wavelength-independent, orientation will be detected in unresolved galaxies. Thus statistical studies of the magnetic fields in distant galaxies will be possible. These same sources are also expected to be excellent background probes in the RM grid.

Within the picture of the turbulent dynamo, the amplitude of the regular magnetic fields in galaxies is expected to decrease at higher redshift. The variation of galactic magnetic field strength with epoch may be reflected in the redshift dependence of the FIR/radio correlation (see Murphy 2009).

\subsubsection{Galaxy clusters}

The ICM of galaxy clusters consists not only of hot thermal gas $\left(T \sim 10^{7}-10^{8} \mathrm{~K}\right)$ emitting in the X-ray domain, but also of magnetic fields and relativistic particles that may give rise to synchrotron radio emission. The most direct evidence for the presence of magnetic fields are the so-called radio haloes and radio relics (see Section 3.4). Other evidence for cluster magnetic fields comes from the Faraday rotation of radio sources that lie within or behind the cluster along our line of sight (e.g. Clarke, Kronberg, \& Bohringer 2001; JohnstonHollitt \& Ekers 2004).

Magnetic fields are thought to play an important role in the development of large-scale structure in the Universe, but their origin and evolution are still poorly understood. Cosmological simulations indicate that the magnetic field strength resulting from the adiabatic compression of the gas falling into the cluster potential well cannot account for more than $1 \%$ of the magnetic field strength that is inferred from observations, so that other amplification mechanisms are required (Dolag et al. 2008). Cosmological simulations also predict that processes related to the cluster formation, such as merger events and shear flows, can in principle amplify the magnetic field up to the observed values. Hence, measurements of the ICM magnetic field, such as the power spectrum and radial profile, are necessary to test these predictions, and to measure the amplification of the magnetic field during cluster formation. Magnetic fields may also affect thermal conduction in the ICM, and so measuring the magnetic field strength 
is crucial to understand the origin of the relativistic particles responsible for the radio halo and relic emission.

\subsubsection{Faraday rotation measures and ICM magnetic fields}

A powerful method to constrain the magnetic field properties in the ICM is to study the Faraday rotation of sources that are within or behind the cluster along our line of sight (e.g. Krause et al. 2009). Recently, Bonafede et al. (2010) have constrained the magnetic field in the Coma cluster by observing seven sources at different projected distances from the cluster centre. The resulting RM images have been compared with the mock RM images obtained with the FARADAY code (Murgia et al. 2004) for different magnetic field configurations. The magnetic field that best reproduces the Coma observations has a central value $B_{0}=4.7 \mu \mathrm{G}$, and declines with the gas density profile $n$ according to $B(r)=$ $n(r)^{0.5}$. Murgia et al. (2004), Govoni et al. (2006), and Vogt \& Enßlin (2005) have achieved similar results on smaller samples. These studies require deep multi-frequency observations of several sources located at different projected distances from the cluster centre, so only a small number of clusters have been studied. The sensitivity of current radio telescopes is insufficient to study a large number of galaxy clusters with many RM probes per cluster, but the next generation of radio telescopes will make this possible.

\subsubsection{Fractional polarisation of radio sources and magnetic field in the ICM}

When synchrotron emission from a cluster or background source crosses the ICM, regions with similar intrinsic direction of the polarisation plane, $\Psi_{\text {int }}$, going through different paths, will be subject to differential Faraday rotation. If the magnetic field in the foreground screen is tangled on scales smaller than the observing beam, radiation with similar $\Psi_{\text {int }}$ but opposite orientation will be averaged out, and the observed degree of polarisation will be reduced (beam depolarisation). In the central region of a cluster, $B$ and $n$ are higher, resulting in a higher RM, and lower fractional polarisation $F_{P}$. Sources at larger radii experience a lower RM, and so suffer from less depolarisation.

Bonafede et al. (2011) selected a sample of massive galaxy clusters and used the NVSS data (Condon et al. 1998) to analyse the polarisation of radio sources as a function of the projected distance from the cluster centre. They find that, statistically, the fractional polarisation decreases with the cluster projected distance. By comparing this trend with that predicted by magnetic field models, they estimate the magnetic field in the cluster centre to be $\sim 5 \mu \mathrm{G}$. The advantage of this approach is that it does not need multi-frequency observations. This effect can be investigated with radio surveys at a single frequency in full polarisation mode. Although such studies do not provide detailed information on magnetic fields in specific clusters, they allow us to understand the average properties of magnetic fields in the ICM, and potentially even on larger scales.

\subsubsection{The origin of cosmic magnetism}

Galaxies and the ICM can only account for about one-third of the baryon density in the local Universe expected from a concordance cosmology. The majority of the missing matter is likely to reside in a warm-hot intergalactic medium (WHIM), which in turn is expected to reside in the cosmic web of the large-scale structure. Detection of the magnetic field found in this cosmic web, or placing stringent upper limits on it, will provide powerful observational constraints on the origin of cosmic magnetism.

Various mechanisms have been suggested for the origin of magnetic fields in our Universe. One possibility is that the fields are truly primordial and that a seed field formed prior to recombination in the very early Universe (Banerjee \& Jedamzik 2003). Alternatively, fields could have been produced via the Weibel instability, a small-scale plasma instability formed at structure formation shocks (Medvedev et al. 2004). Both of these mechanisms occurred early in cosmic history, prior to galaxy formation, and are therefore referred to as early-type mechanisms. In contrast, possible late-type mechanisms are processes where the field was injected into the WHIM via the action of jets from AGNs and other outflows such as galactic winds (e.g. Kronberg 2004).

The SKA pathfinder polarisation surveys will together generate a rich data set from which a wide, high-density, broad bandwidth RM grid may be built, and weak magnetic fields in intergalactic filaments may be detected for the first time. A lower limit for the intergalactic magnetic field has been determined ( $10^{-15} \mathrm{G}$; Dolag et al. 2011).

In the filamentary structures of the cosmic web, field strengths are expected to be far more sensitive to the origin of the magnetic fields than in larger gravitationally bound structures where turbulent amplification will have led to saturation of the field. Specifically, the strength and penetration of magnetic fields in filaments is expected to be much lower if the field arises from a 'late' astrophysical source (AGNs, galactic winds, etc.), compared with that arising from a primordial one, and simulations have demonstrated that the strength of these turbulently amplified early-type seed fields range typically between 0.1 and $0.01 \mu \mathrm{G}$ (Ryu et al. 2008). Consequently, observations of the filamentary cosmic web are invaluable for inferring the origin of cosmic magnetism. Although the predicted field strengths are extremely low, such RMs are observationally accessible with the SKA pathfinder telescopes.

In addition, the rotation of emission from background radio sources through the large-scale structure filaments within e.g. superclusters of galaxies can also be used to infer the origin of cosmic magnetism. A statistical analysis of these RMs to measure the power spectrum of the magnetic field of the cosmic web (Kolatt 1998), using cross-correlations with other large-scale structure indicators, will also provide stronger constraints than individual RMs. These measurements will also probe the distribution and density of thermal gas at the interface between clusters and the cosmic web, 
which has important consequences for a number of related areas in astrophysics and will be highly complementary to X-ray and Sunyaev-Zel'dovich studies of these structures. However, because of the low temperatures of these regions, RM measurements may provide the only way to probe them.

\subsubsection{High-redshift rotation measures}

Studies of high-redshift objects (e.g. Kronberg et al. 2008, who examined the redshift dependence of the spread of RMs) suggest that the amplification of magnetic fields occurred more rapidly than the expectations of mean-field dynamo theory. The conclusions of this type of work are so far limited by small samples, together with uncertainties in the removal of the Milky Way foreground RM structure. SKA pathfinders will provide RM grids that will enable this kind of study to be done with significantly lower uncertainty. A better comparison with the predictions of dynamo theory (see e.g. Arshakian et al. 2009) will thus become possible.

\subsection{Cosmology}

The SKA pathfinder surveys (here referred to as SPARCS surveys) will not only be an important preparation for SKA surveys, but will also be able to deliver important cosmological results themselves. Earlier surveys were able to provide valuable constraints on cosmology and cosmic evolution (e.g. Blake \& Wall 2002; Overzier et al. 2003). Here we show that SPARCS surveys will be able to constrain cosmological models and parameters via several probes: the ISW effect (Sachs \& Wolfe 1967), the lensing effect known as cosmic magnification, and source count correlations.

The source count correlation function (or if measured in Fourier space, the power spectrum) is a well-known statistic measuring the excess probability that sources are clustered together on different scales. In the radio surveys considered here, we assume that individual redshifts will not be available, so the power spectrum will be measured on the 2D celestial sphere, by measuring the spatial auto-correlation function of the radio sources in the SPARCS surveys.

Cosmic magnification is a gravitational lensing effect, where the number density of background sources is affected by foreground structures in two competing ways. First, lensing dilates the background patch of sky, reducing the background number density. Second, the lensing expands the size of background sources while maintaining their surface brightness, resulting in an increase of apparent luminosity of sources, increasing the number density above any given detection threshold. Either of these effects can dominate depending on the luminosity function, so there may be either a correlation or anti-correlation of background number density with foreground structures. The effect is sensitive to the geometry of the Universe and the growth of structures in the Universe. In our case, we correlate background SPARCS radio sources with foreground sources from shallower optical surveys, such as SDSS (Abazajian et al. 2009) for northern surveys, Skymapper (Keller et al. 2007) or DES (The Dark Energy Survey Collaboration 2005) for southern surveys.

The ISW effect is a gravitational redshift, due to the evolution of the gravitational potential while photons pass through under- or overdensities in their path from the last scattering surface to us. In an Einstein-de Sitter universe, the blueshift of a photon falling into a well is cancelled by the redshift as it climbs out. But in a cosmology where there are evolving gravitational potentials, photons will experience a net blueor redshift, leading to a net change in photon temperature, which accumulates along the photon path. This translates into CMB temperature anisotropies proportional to the variation of the gravitational potentials:

$$
\frac{\delta T}{T} \propto \int[\dot{\Psi}(z)+\dot{\Phi}(z)] \mathrm{d} z
$$

Here, we have introduced the gravitational potentials $\Psi$ and $\Phi$, which are the time-part and space-part perturbations of the Friedmann-Robertson-Walker (FRW) background in the conformal Newtonian gauge, $\mathrm{d} s^{2}=-a^{2}\left((1+2 \Psi) \mathrm{d} \tau^{2}-\right.$ $\left.(1-2 \Phi) \mathrm{d} x^{2}\right)$. In GR, in the absence of anisotropic stress, we expect $\Psi$ and $\Phi$ to be identical.

The ISW effect can contribute significantly to the CMB temperature fluctuations on large angular scales, but it enhances only the low $\ell$ multipoles, and is smaller than other CMB anisotropies. For this reason, in order to detect the effect, we have to cross-correlate $\mathrm{CMB}$ temperature maps with tracers of the potential wells (Crittenden \& Turok 1996) such as galaxies; if the potential evolves, then we should observe a correlation between CMB temperature anisotropies and the galaxy distribution. For the tests considered here, we crosscorrelate the SPARCS radio sources with the CMB data from WMAP or Planck.

\subsubsection{Calculating probe predictions}

Here we consider the measurements of the three probes discussed above (source count correlations, cosmic magnification, and ISW) and calculate the resulting constraints obtained with the LOFAR, WODAN, and EMU surveys (see Raccanelli et al. 2011 for details about the surveys and the assumed distributions and bias).

To predict the redshift distribution for these surveys, we use the latest empirical simulations developed for the SKA continuum survey (Wilman et al. 2008). This simulation provides different prescriptions for the redshift evolution of the various populations which dominate the radio source counts at five different radio frequencies: 150, 610, 1400,4860 , and $18000 \mathrm{MHz}$. Catalogues are generated from the $S^{3}$ database, ${ }^{3}$ using the radio flux-density limits of different surveys, and we perform our analysis by extracting a catalogue from the database with cuts at the $10 \sigma$ detection levels of the forthcoming surveys.

The $S^{3}$ simulation provides us with a source catalogue in which sources are identified by type (e.g. SB, SFG, FRI, FRII,

\footnotetext{
${ }^{3}$ http://s-cubed.physics.ox.ac.uk
} 


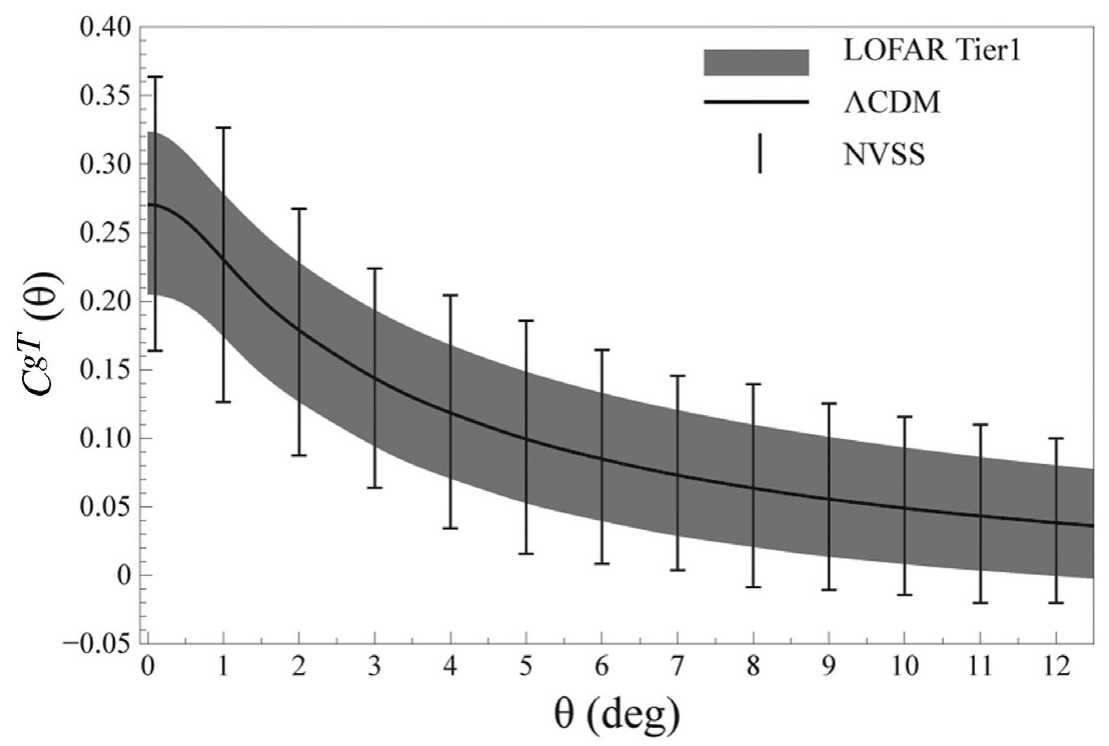

Figure 20. Predicted correlations of the CMB with the LOFAR Tier 1 survey data. As a comparison, we also show error bars from current NVSS measurements. Note the improvement we obtain with one of the pathfinders over current constraints on all scales.

and RQQ). The bias is computed separately for each galaxy population using the formalism of Mo \& White (1996), in which each population is assigned a dark matter halo mass chosen to reflect the observed large-scale clustering. With this framework, the increasing bias $b(z)$ with redshift would lead to excessively strong clustering at high redshift, so the bias for each population is held constant above a certain cut-off redshift (see Wilman et al. 2008, for details).

We calculate the angular power spectra for each of the probes, i.e. source-source, radio background-optical foreground, and source CMB. Each of these is a projection of the density power spectrum, see Raccanelli et al. (2011) for details. For instance, the ISW cross-power spectrum is given by

$$
C_{\ell}^{g T}=\left\langle a_{\ell m}^{g} a_{\ell m}^{T *}\right\rangle=4 \pi \int \frac{\mathrm{d} k}{k} \Delta^{2}(k) W_{\ell}^{g}(k) W_{\ell}^{T}(k)
$$

where $W^{g}{ }_{\ell}$ and $W^{T}{ }_{\ell}$ are the galaxy and CMB window functions, respectively, and $\Delta^{2}(k)$ is the logarithmic matter power spectrum today.

The galaxy window function, which enters all three power spectra, is written as

$$
W_{\ell}^{g}(k)=\int \frac{\mathrm{d} N}{\mathrm{~d} z} b(z) D(z) j_{\ell}[k \eta(z)] \mathrm{d} z
$$

where $(\mathrm{d} N / \mathrm{d} z) \mathrm{d} z$ is the mean number of sources per steradian with redshift $z$ within $\mathrm{d} z$, brighter than the flux limit, $b(z)$ is the bias factor relating the source to the mass overdensity, $D(z)$ is the linear growth factor of mass fluctuations, $j_{\ell}(x)$ is the spherical Bessel function of order $\ell$, and $\eta(z)$ is the conformal lookback time. Similar window functions exist for the CMB and foreground elements for the different power spectra.
We can write corresponding correlation functions as a function of the angular separation $\theta$ :

$$
C(\theta)=\sum_{\ell} \frac{2 \ell+1}{4 \pi} C_{\ell} L_{\ell}(\cos \theta),
$$

where $L_{\ell}$ are the Legendre polynomials of order $\ell$.

\subsubsection{Predictions for the pathfinders}

We have calculated correlation functions or power spectra for the ISW, cosmic magnification, and source counts for the forthcoming radio surveys. For example, in Figure 20 we show our result for the CMB-LSS cross-correlation using the predicted redshift distributions and bias for the LOFAR Tier 1 survey, where we assume the standard $\Lambda \mathrm{CDM}+\mathrm{GR}$ model and the shaded areas are cosmic-variance errors, calculated via

$$
\sigma_{C_{\ell}^{g T}}=\sqrt{\frac{\left(C_{\ell}^{g T}\right)^{2}+C_{\ell}^{g g} C_{\ell}^{T T}}{(2 \ell+1) f_{\text {sky }}}},
$$

where $f_{\text {sky }}$ is the sky coverage of the survey; $C^{g g}{ }_{\ell}$ and $C^{T T}{ }_{\ell}$ are the auto-correlations density-density and temperaturetemperature, and contain shot noise and detector noise; the errors are then converted to real space as

$$
\sigma^{2}(\theta)=\sum_{\ell}\left(\frac{2 \ell+1}{4 \pi}\right)^{2} P_{\ell}^{2}(\cos \theta) \sigma_{C_{\ell}^{g T}}^{2}
$$

Now we wish to predict the combined cosmological measurements that the SKA pathfinders should allow. In order to do this, we perform a joint analysis including $\mathrm{CMB}+\mathrm{SNIa}$ priors, ISW, cosmic magnification, and the auto-correlation of radio sources. We test deviations from the standard 
$\Lambda \mathrm{CDM}+\mathrm{GR}$ model for two cases: we first parameterise modifications of the theory of gravity; and then alternatively, we assume GR to be the correct model to describe gravity and consider a dynamical dark energy model.

\subsubsection{Modified gravity constraints}

We want to test deviations from GR using the $\{\eta, \mu\{$ parameters, which are defined via

$$
\begin{gathered}
\frac{\Phi}{\Psi}=\eta(a, k), \\
k^{2} \Psi=-4 \pi G a^{2} \mu(a, k) \rho \Delta,
\end{gathered}
$$

where $\Delta$ is the gauge-invariant comoving density contrast:

$$
\Delta=\delta+3 \frac{a H}{k} v
$$

in General Relativity $\eta(a, k)=\mu(a, k)=1$, while in alternative models they deviate from this value, and can also be functions of time and scale (e.g. Zhao et al. 2010).

We model the time evolution of $\mu$ and $\eta$ as (see Zhao et al. 2010 for details)

$$
\begin{aligned}
& \eta(z)=\frac{1-\eta_{0}}{2}\left(1+\tanh \frac{z-z_{s}}{\Delta z}\right)+\mu_{0}, \\
& \mu(z)=\frac{1-\mu_{0}}{2}\left(1+\tanh \frac{z-z_{s}}{\Delta z}\right)+\mu_{0} .
\end{aligned}
$$

In Figure 21 (top panel), we show predictions for how well we can constrain the modified gravity parameters using the EMU survey. Also displayed are the $68 \%$ confidence level for current surveys (SNe, WMAP, and SDSS; Raccanelli et al. 2011). We use the current best-fit model as our fiducial model; note that if this current best fit remains the best fit, the standard GR+LCDM model would be excluded with forthcoming radio surveys at high significance. In any case, the surveys impressively reduce the permitted parameter space.

\subsubsection{Constraints on dark energy evolution}

To study how well the SKA pathfinders will constrain the evolution of dark energy we use the $\left\{w_{0}, w_{a}\{\right.$ parameterisation (Linder 2003) of the dark energy equation of state:

$$
w(a)=w_{0}+w_{a}(1-a) .
$$

In Figure 21 (bottom panel) we show predictions for the constraints on the parameters $\left\{w_{0}, w_{a}\{\right.$, using the EMU survey. Again, the constraints are substantially improved over the currently permitted region of parameter space.

\subsubsection{Bias}

To obtain constraints on cosmological parameters from continuum surveys, the redshift distribution and bias of radio sources needs to be known. Here we suggest a method for measuring the bias of radio sources at high redshift. We cross-match FIRST radio sources (Becker et al. 1995) with
SDSS-DR7 data (Abazajian et al. 2009) and use photometric redshifts in the DR7 catalogue to obtain an estimate of the redshift distribution of the optically identified sources. To probe the high- $z$ radio continuum population, we assume that the $z$-distribution of all FIRST sources is given by the SKADS simulation (Wilman et al. 2008) and we subtract off the distribution of the optically identified sources to estimate the $z$-distribution of the unidentified sources. By measuring the angular correlation function of the unidentified sources and comparing this with predictions for dark matter clustering, we will then be able to estimate the bias at $z \sim 0.7$. Some initial results using this technique are presented in Passmoor et al. (2013).

If SKA pathfinder surveys are to be used to measure cosmological parameters, it will be important to continue and extend this work to obtain a better estimate of bias for different classes of radio source, and also to understand the sensitivity of the derived cosmological parameters to uncertainty in the value of the bias parameter.

\subsubsection{The effect of redshifts on cosmological tests}

The cosmology discussion to this point makes the conservative assumption that no redshifts are available for individual radio sources, although we do know the redshift distribution of the different classes of source, and the already impressive results shown in Figure 21 are calculated using this assumption. While less than about $1 \%$ of radio sources in SPARCS surveys are likely to have spectroscopic redshifts at the time of the data releases, photometric and statistical redshift information is likely to be available for $\sim 50 \%$ of sources, as described in Section 4.7.

Camera et al. (2012) have explored the impact of photometric redshifts on the cosmological measurements from EMU and WODAN. Using only the galaxy clustering angular power spectrum and dark energy constraints, they find that even approximate photometric redshifts obtained from SDSS and SkyMapper yield a significant improvement to the constraints provided by the radio data alone.

An alternative approach has been suggested by Norris et al. (2011b), using radio data alone. Sources with detected polarisation in the POSSUM survey (see Section 2.4) are virtually all AGNs, with an expected median redshift of $\langle z\rangle \sim 1.8$, while the remaining unpolarised EMU sources will be a mixture of AGNs and SFGs, with an expected median redshift of $\langle z\rangle \sim 1$.1. The unpolarised sources can therefore be treated as a separate population from the polarised sources for the purposes of the cosmological tests described above, giving two radio source samples at different redshift ranges, thus potentially yielding a measurement of the time-dependent component of the dark energy and modified gravity parameters. However, modelling is required to establish whether this technique yields a significant improvement over other techniques. 

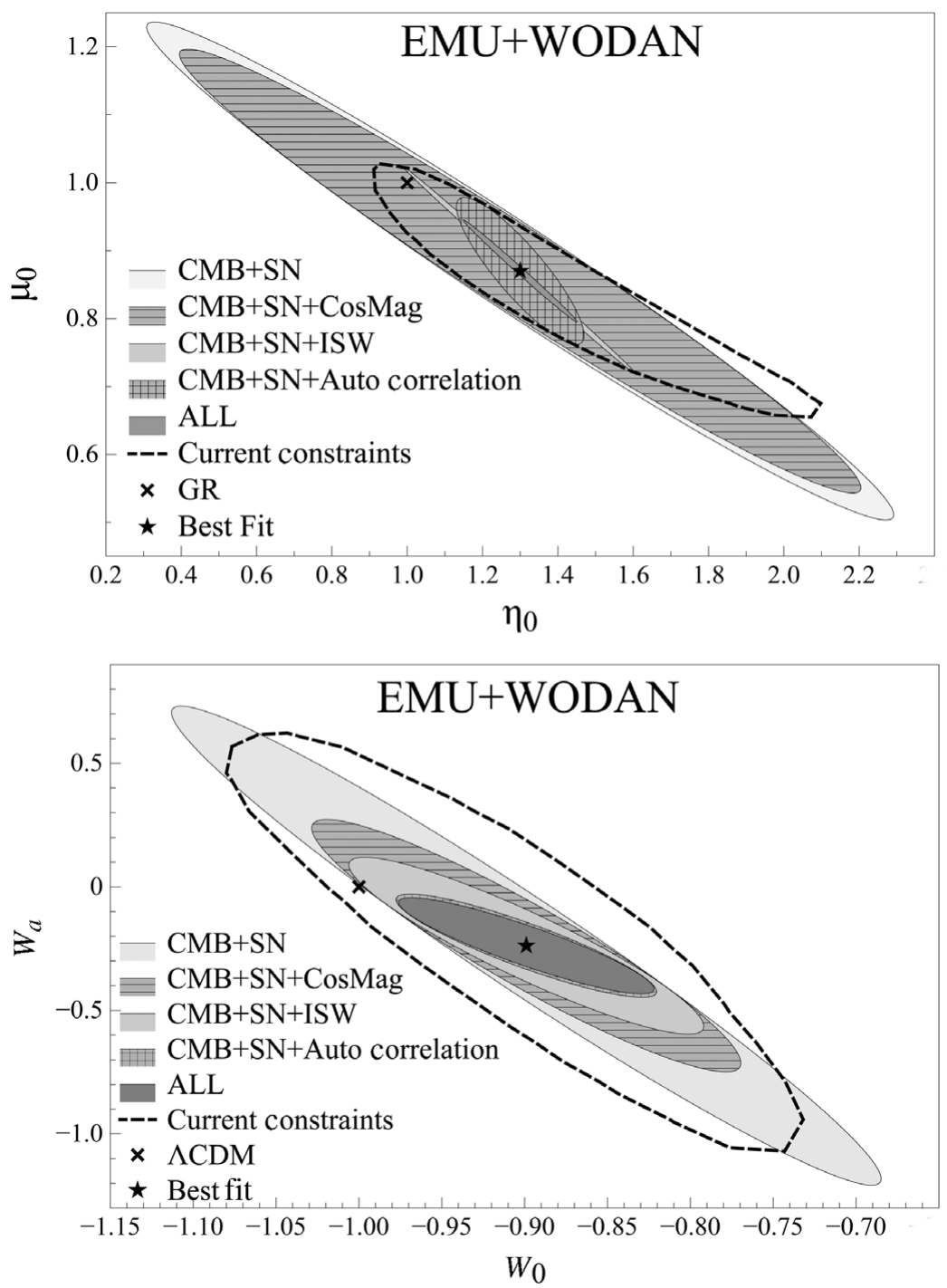

Figure 21. Predicted constraints on parameters using EMU+WODAN. The outer dotted ellipse shows the current constraints and the innermost grey ellipse shows the constraints available using EMU+WODAN with the three probes described. Bottom panel: dark energy parameters, showing current and predicted $68 \% \mathrm{CL}$ constraints. Top panel: modified gravity parameter constraints.

\subsubsection{Weak lensing}

Weak gravitational lensing by large-scale dark matter in the Universe distorts the images of faint background galaxies. Measurements of this 'cosmic shear' effect as a function of redshift can provide powerful constraints on dark energy and its evolution over cosmic time (e.g. Albrecht et al. 2006; Peacock et al. 2006). The measurements require a large number of well-resolved galaxies to reduce the 'shape noise' associated with the intrinsic variety of galaxy shapes. Most cosmic shear studies to date have therefore been at optical wavelengths. Radio measurements have typically been less effective because of smaller number densities and poorer spatial resolution. Chang, Refregier, \& Helfand (2004), however, made a statistical detection of cosmic shear in the VLA FIRST survey, and Patel et al. (2010) used VLA and MERLIN data to explore radio weak lensing techniques.

Measuring weak lensing at radio wavelengths potentially offers a number of advantages over optical surveys in terms of minimising instrumental and astrophysical systematic effects. For example, radio telescopes have highly stable and well-understood point-spread functions (PSFs), which makes them particularly suited for measuring the small distortions caused by weak lensing. In addition, a future weak lensing survey conducted with the SKA could yield redshifts for a significant fraction of the lensed galaxies through the detection of their $\mathrm{H}$ I emission lines (e.g. Blake et al. 2007). Uncertainties and biases associated with photometric redshift errors would consequently be greatly reduced with an SKA lensing survey. 
A major astrophysical cause of systematic error in weak lensing measurements is the intrinsic alignment in galaxy shapes, caused by tidal torquing during the galaxy formation process. Cleanly separating these from the extrinsic alignments caused by weak lensing will be crucial for interpreting precision weak lensing measurements in the future. Brown \& Battye (2011a) showed that polarisation, which is routinely measured at radio wavelengths can, in principle, discriminate between the lensing distortions and intrinsic alignments.

Blake et al. (2007) have explored the potential of the SKA to perform a precision weak lensing survey. The potential advantages described above would make such a survey highly complementary to future large-scale optical lensing surveys. The SKA pathfinders offer excellent opportunities to develop the field of radio weak lensing and to demonstrate the above techniques and advantages on real data. For example, largearea surveys with arcsec resolution with LOFAR can test weak lensing techniques on large radio data sets. Moreover, the $e$-MERLIN telescope offers an exciting opportunity to perform deep sub-arcsec radio imaging over a large FOV which will be an ideal testbed for investigating radio lensing techniques. This potential has recently been recognised with the award of $\sim 800 \mathrm{~h}$ of $e$-MERLIN legacy survey time for the Supercluster Assisted Shear Survey (SuperCLASS), which will image a $1.75 \mathrm{deg}^{2}$ region of sky containing a supercluster to a depth of $4 \mu \mathrm{Jy}$ beam $^{-1}$ with 0.2 -arcsec resolution, including polarisation information. This study is expected to detect $\sim 1-2$ galaxies per square arcmin, which will be used to map the dark matter distribution in the vicinity of the supercluster (Brown \& Battye 2011b).

\subsection{Variability and transients}

Transient and variability studies are key science drivers for SKA pathfinder instruments, and potentially for the SKA as well. Here we briefly discuss some of the key issues that need to be considered and addressed on the road to the SKA, potentially via pathfinder instruments. We also highlight some of the outcomes that can potentially be achieved through future surveys.

Blind and dedicated radio transient surveys can potentially

- discover new classes of energetic objects ('unknownunknowns'),

- monitor known transient and variable radio sources ('known-knowns'),

- search for previously undetected transient and variable sources ('known-unknowns'),

- explore the intervening medium via extreme scattering events, and

- search for a SETI signal.

Table 1 summarises our current knowledge of variable radio sources (see also Condon et al. 1979 and references therein), showing observations at a variety of cadences, frequencies, and Galactic latitudes. A complex variety of vari- ability is reported; in some cases short timescale variations at low frequencies are attributed to interstellar scintillation, while long-term variations at $\mathrm{GHz}$ frequencies are in some cases attributed to AGN activity. A systematic experiment to map the surface density of variable radio sources as a function of flux, frequency, and cadence would be an invaluable step on the road to the SKA.

Source variability (including bright calibrators; see Gaensler \& Hunstead 2000) may affect the calibration and clean strategies required for future surveys and will ultimately impact on the final dynamic range and image fidelity (e.g. see Stewart, Fenech, \& Muxlow 2011). Therefore, characterising the variability and abundance of known radio sources is a necessity for planning future surveys and calibration strategies. Many of the compact nJy sources to be studied by the SKA will be affected by scintillation (Dennison \& Condon 1981; Rickett 1986). Furthermore, at these sensitivities highly variable sources in the range $1 \mu \mathrm{Jy}-1 \mathrm{mJy}$ will yield a dynamic range of 1 000:1 (and greater). Carilli, Ivison, \& Frail (2003) report a surface density of highly variable sources of up to $18 \mathrm{deg}^{-2}$ (see Table 1) at a detection threshold of $\sim 0.1 \mathrm{mJy}$ : such a high abundance could seriously impact SKA surveys.

Possible transient sources range from extrasolar planets at frequencies below $50 \mathrm{MHz}$ (Grießmeier, Zarka, \& Spreeuw 2007) to brown dwarf flares at $8 \mathrm{GHz}$ (Berger 2002). Early blind searches for transient sources suggest that a bright $(\geq 0.1 \mathrm{Jy})$, frequent, population of $\mathrm{GHz}$ transients does not exist (see e.g. Croft et al. 2010, 2011; Bower et al. 2010; Bower \& Saul 2011; Bell et al. 2011). The benchmark survey of Bower et al. (2007) which predicted a substantial number of radio transients in the sub-mJy range has recently been revised by Frail et al. (2012), lowering the predicted surface density of sources. An all-sky survey could however still potentially detect several thousand new transients, though a much lower yield would also be consistent with the current uncertainties (see e.g. Bannister et al. 2011; Murphy et al. 2012).

So far many surveys have been conducted in the $\mathrm{GHz}$ regime, at low frequencies, synchrotron sources may be optically thick with lower peak fluxes. Coherent processes therefore might dominate transient detections below $1 \mathrm{GHz}$, and rate estimates should differentiate between the two processes. Exploring the transient rates at low frequencies is an especially important goal for pathfinders such as LOFAR and the LWDA (Fender, Wijers, \& Stappers 2008; Lazio et al. 2010) and ultimately SKA-Low.

A goal for the transient key science projects is to sample a given field on a logarithmic range of timescales, perhaps commensally with a project revisiting a given field at a variety of timescales to build up the necessary sensitivity. A further goal of the variability studies will be to follow up on objects (such as pulsars or radio stars) which may be detected in the large continuum surveys, and may be manifested in the image as having spoke-like features, as discussed in Section 3.8.4. When the EMU and WODAN surveys start, transient 
Table 1. A selection of variable source statistics taken from the literature. $\rho$ gives the snapshot rate of sources $\left(\mathrm{deg}^{-2}\right) . t_{\mathrm{char}}$ gives the characteristic timescale on which the variability was sampled. $\Delta S / S$ gives the fractional change in flux (please refer to individual publications for further details).

\begin{tabular}{llllll}
\hline \hline Study & Flux (mJy) & $\rho\left(\mathrm{deg}^{-2}\right)$ & $t_{\text {char }}$ & $v(\mathrm{GHz})$ & $\Delta S / S$ \\
\hline Bannister et al. (2011) & $>14$ & 0.268 & days-years & 0.843 & $\geq 50 \%$ \\
Carilli et al. (2003) & $>0.1$ & $<18$ & 19 days and 17 months & 1.4 & $\geq 50 \%$ \\
Becker et al. (2010) & $>0.1$ & 1.6 & $\sim 15$ years & 4.8 & $\geq 50 \%$ (d) \\
Frail et al. (2003) & $>0.25$ & 5.8 & $\sim 1$ day & 5 and 8.5 & $\geq 50 \%$ \\
Gaensler \& Hunstead (2000) & $>2500$ & - & days-years & 0.843 & $<20 \%$ (d) \\
Condon \& Backer (1975) & $\sim 1000-25000$ & - & days & 2.695 and 8.085 & $0.5 \%$ and $0.98 \%$ (a) \\
Dennison et al. (1981) & $\sim 500-33000$ & - & 5-10 years & 0.318 & $8 \%-100 \%$ \\
Simonetti \& Cordes (1990) & $400-12000$ & - & days & 0.820 and 1.41 & $4.1 \%$ and $3.5 \%$ (b) \\
Ryle et al. (1978) & $200-2000$ & - & months & $2.7-15.4$ & $10 \%-50 \%(\mathrm{c})$ and (d) \\
Taylor \& Gregory (1983) & $18-1200$ & - & days-months & 5 & $10 \%-400 \%$ (c) and (d) \\
\hline \hline
\end{tabular}

(a) $0.5 \%$ at $2.695 \mathrm{GHz}$ and $0.98 \%$ at $8.085 \mathrm{GHz}$. Values are derived from the average (daily) fractional change in 16 sources.

(b) $4.1 \%$ at $0.820 \mathrm{GHz}$ and $3.5 \%$ at $1.41 \mathrm{GHz}$. Values are derived from the average modulation index ( $\mathrm{rms} / \mathrm{mean}$ ) in 13 flat spectrum sources.

(c) Also see Rickett (1986).

(d) Observations in the direction of the Galactic plane.

and variability studies through projects such as VAST (Chatterjee et al. 2010; Murphy et al. 2012) can be performed commensally via comparison with previous catalogues such as SUMSS (Bock, Large, \& Sadler 1999) and NVSS (Condon et al. 1998). Through commensal and targeted observations a more complete census of the abundance of transients and variables can be achieved prior to SKA operations.

\subsection{Galactic science}

The proposed wide-field surveys such as EMU and POSSUM include the Galactic plane, giving the opportunity to create a sensitive atlas of discrete Galactic radio continuum sources. Most of these sources represent the interaction of stars at various stages of evolution with their environment. The high sensitivity of the surveys allows access to all stages of $\mathrm{H}$ II regions from the optically thick hyper-compact kind presumably related to the earliest stages of a 'turned-on' star to large $\mathrm{H}$ II regions related to massive mature or giant stars producing strong stellar winds. Due to the high resolution of these surveys we can detect the youngest, most compact supernova remnants and pulsar wind nebulae. We can detect and study planetary nebulae giving us insight in the late stages of the evolution of low-mass stars. And we can detect radio stars and pulsars. High-sensitivity polarisation surveys such as POSSUM will also open the door to the identification of currently unexplained polarisation features known as Faraday screens.

At $1.4 \mathrm{GHz}$, the main mechanisms for radio emission from discrete Galactic objects are thermal free-free emission from $\mathrm{H}$ II regions and planetary nebulae and non-thermal synchrotron emission from supernova remnants and pulsar wind nebulae. Resolved Galactic sources can be separated by their spectral and linear polarisation properties. Young $\mathrm{H}$ II regions such as compact, ultra-compact, and hyper-compact sources are optically thick at frequencies around $1.4 \mathrm{GHz}$, and thus easy to separate from not only Galactic but also extragalac- tic sources even if they are not resolved. Large evolved H II regions have flat optically thin spectra compared with supernova remnants that may show similar structures but have steeper spectra, and are typically linearly polarised. Pulsar wind nebulae also have flat radio spectra, but are linearly polarised. Comparisons with surveys at other wavelength such as infrared or X-ray will further help to discriminate individual sources of interest for follow-up studies.

\subsubsection{H II regions}

The high sensitivity of the planned wide-field 1.4-GHz surveys such as EMU and WODAN allows access to all stages of the evolution of $\mathrm{H}$ II regions, even though free-free emission near $1.4 \mathrm{GHz}$ will be optically thick for the very dense ultra- and hyper-compact $\mathrm{H}$ II regions (UCHII and HCHII, respectively), which are too small to be resolved by a 10arcsec beam, unless they are very nearby. UCHII and HCHII represent a very young phase in the development of an $\mathrm{H}$ II region (Churchwell 2002).

Expected flux densities at $1.4 \mathrm{GHz}$ for the most compact $\mathrm{H}$ II regions are displayed in Figure 22. The separation between ultra-compact and hyper-compact $\mathrm{H}$ II regions is at a diameter of about $0.02 \mathrm{pc}$. Hence, EMU will be able to detect all ultra-compact $\mathrm{H}$ II regions and also many hyper-compact objects. There is still confusion about what a hyper-compact $\mathrm{H}$ II region actually is. Are they just extremely compact ultracompact $\mathrm{H}$ II regions or a new class of objects? There is evidence that HCHIIs have somewhat different spectral characteristics than the UCHIIs, which might be explained by a density gradient within the ionised gas (Kurtz 2005). So far only a small number of HCHIIs are known. A more complete census of these objects with a sensitive wide-field survey is required to get a more complete picture of the evolutionary process of $\mathrm{H}$ II regions. Follow-up observations at higher radio frequencies or a comparison with high radio frequency surveys is then required to determine physical characteristics which will help to unravel the true nature of these objects. 


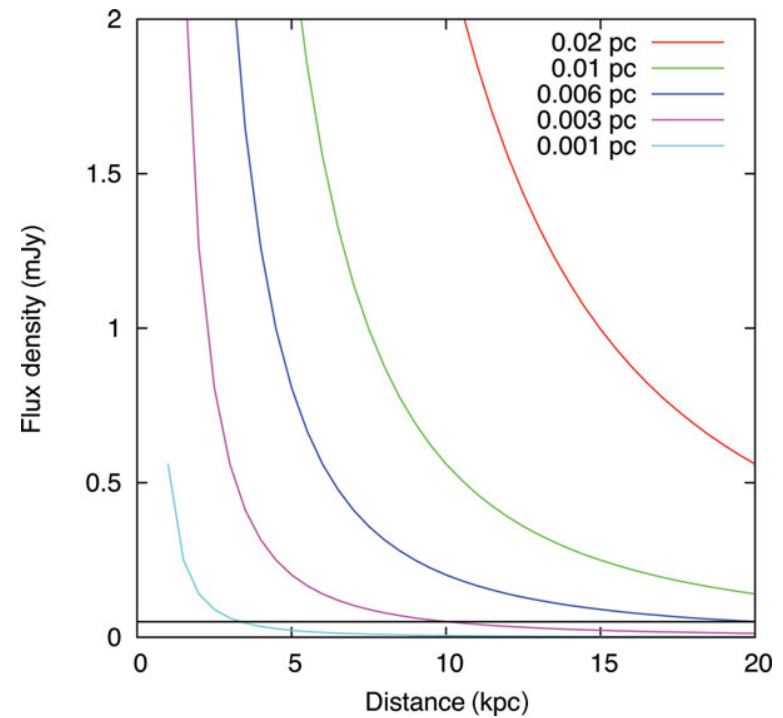

Figure 22. Predicted peak radio flux density of unresolved UCHIIs and HCHIIs at $1.4 \mathrm{GHz}$ as a function of distance from the sun. UCHIIs and HCHIIs with different diameters are displayed. We assumed a constant electron temperature of $8000 \mathrm{~K}$, a homogeneous distribution of material, and high optical depth $(\tau \gg 1.0)$.

We can also probe the magnetic field inside $\mathrm{H}$ II regions by either studying the RM signature of polarised point sources 'shining through' the object (e.g. Harvey-Smith, Madsen, \& Gaensler 2011) or by studying overlapping or even related diffuse polarised emission (e.g. Foster et al. 2006).

\subsubsection{Planetary nebulae}

Planetary nebulae $(\mathrm{PNe})$ are the most abundant compact Galactic sources in the NVSS (Condon et al. 1998). Condon \& Kaplan (1998) identified 680 NVSS radio sources brighter than $2.5 \mathrm{mJy}$ with planetary nebulae. Assuming a detection limit of $50 \mu \mathrm{Jy}$, EMU will be able to detect unresolved PNe up to seven times farther away than NVSS, potentially leading to a significant increase of the number of PNe detected at radio frequencies. PNe can be very useful tools for measuring extinction and probing SFRs of stars not massive enough to produce supernovae or H II regions (Condon \& Kaplan 1998; Condon, Kaplan, \& Terzian 1999). Identification of these PNe, however, will be difficult.

The ionised low-density circumstellar material of nearby planetary nebulae, released during the Asymptotic Giant Branch (AGB) wind phase of the central stellar object, is illuminated by background linear polarised emission through Faraday rotation. Free thermal electrons embedded in a magnetised medium rotate background linear polarisation emission to create an RM signature observable at $1.4 \mathrm{GHz}$. A typical radio telescope operating in this wavelength range proves to be more sensitive to the Faraday rotation of a plasma region than to its bremsstrahlung signature. Linear polarisation studies of nearby planetary nebulae can reveal important information about the mass loss history of old AGB stars or white dwarfs that cannot be obtained easily any other way (e.g. Ransom et al. 2008, 2010).

\subsubsection{Supernova remnants}

Only 274 supernova remnants (SNRs) have been discovered so far in our Galaxy (Green 2009). ${ }^{4}$ However, the estimated population of SNRs is much higher (maybe 500-1 000; Helfand et al. 2006). This discrepancy is mainly caused by a strong bias towards bright extended objects. These are the mature SNRs expanding into a medium- to high-density environment. There are two groups of missing SNRs. The compact, presumably very young, objects were missed by previous wide-field surveys, because they are either confused by other nearby objects or mistaken for extragalactic sources if they are not resolved. The second group of missing SNRs are low surface brightness SNRs. A population of more than 1000 SNRs for our Galaxy can also be extrapolated using the Canadian Galactic Plane Survey (CGPS; Taylor et al. 2003) and its supernova remnant catalogue (Kothes et al. 2006). The CGPS is a radio continuum and $\mathrm{H}$ I survey of the Outer Galaxy. Since the distance to the edge of the Galaxy, and hence to every object in the survey, is very short, the $\sim 1$ arcmin resolution at $1420-\mathrm{MHz}$ radio continuum ( $\leq 1 \mathrm{pc})$ is sufficient to resolve any SNR - even those which are very young.

EMU and POSSUM will have a very similar spatial resolution than the CGPS but much higher sensitivity. The 10arcsec beam translates to about the same spatial resolution at the most distant edge of the Galaxy through the inner part of the Milky Way $(\approx 20 \mathrm{kpc})$ than the CGPS beam does towards the Outer Galaxy. Hence, we would expect to detect a similar number density of SNRs. This indicates that EMU in conjunction with POSSUM could discover more than 500 new SNRs. Resolved SNRs can be easily distinguished from extragalactic sources and Galactic $\mathrm{H}$ II regions by an analysis of structure and spectral and polarisation signatures.

Radio polarisation observations of SNRs can also be a powerful tool to probe the magnetic field into which these objects are expanding. SNRs act as magnifying glasses of ambient magnetic fields, since they freeze and compress it into the expanding shell of the swept up material. Radio polarisation and RM studies can reconstruct the 3D ambient magnetic field, as demonstrated in the studies by Uyanıker, Kothes, \& Brunt (2002), Kothes \& Brown (2009), and Harvey-Smith et al. (2010). Radio polarisation studies of SNRs with EMU and POSSUM can probe the large-scale magnetic field of the Inner Galaxy, detect potential field reversals, and probe the transition from the Galactic plane to the halo.

\subsubsection{Pulsars and their wind nebulae}

Pulsars generate magnetised relativistic particle winds, inflating an expanding bubble called a pulsar wind nebula (PWN), which is confined by the expanding supernova ejecta. Electrons and positrons are accelerated at the termination shock

\footnotetext{
${ }^{4}$ http://www.mrao.cam.ac.uk/surveys/snrs/snrs.info.html
} 
some $0.1 \mathrm{pc}$ distant from the pulsar and interact with the magnetic field to produce synchrotron emission across the entire electromagnetic spectrum. At $\mathrm{GHz}$ frequencies, the flat spectrum PWNe stand out from steep spectrum SNRs and can be distinguished from $\mathrm{H}$ II regions by their linear polarisation signal.

The high resolution and sensitivity of the proposed widefield 1.4-GHz surveys will enable us to built a more complete census of Galactic pulsar wind nebulae down to unprecedented levels.

The number of pulsars at high Galactic latitudes is poorly constrained since most surveys concentrate on a narrow band along the Galactic plane. Out of the 1433 pulsars with measured $1400-\mathrm{MHz}$ fluxes listed in the ATNF Pulsar Catalogue (Manchester et al. 2005), 1410 have integrated fluxes above $50 \mu \mathrm{Jy}$. Only 310 of those have Galactic latitudes $|b|>5^{\circ}$. Sensitive high-resolution radio continuum surveys will enable us to get a more complete picture of the pulsar distribution in the Milky Way and their RMs will help to constrain the configuration and strength of the large-scale Galactic magnetic field.

Pulsars should be distinguishable from other point-like radio sources because of their steep radio continuum spectra, their polarisation, and artefacts caused by short-term variability. These observational signatures will be complemented by the variability and transient surveys discussed in Section 3.7.

\subsubsection{Radio stars}

Radio stars emit a tiny fraction of their total luminosity in the radio band. For example, the quiet Sun has a radio luminosity of $10^{14} \mathrm{~W}$, which is only $\sim 10^{-12}$ of its bolometric luminosity. Nevertheless, in many cases, radio observations of stars and stellar systems have revealed astrophysical phenomena, not detectable by other means, that play a fundamental role in our understanding of stellar evolution and of physical processes that operates in stellar atmospheres.

Generally, the brightest stellar radio emission appears to be associated with magnetically induced phenomena, such as stellar flares, related to the presence of a strong and/or variable stellar magnetic field (high brightness temperature) or with enhanced mass loss (large emitting surface).

Among the brightest radio sources are active stars and binary systems, including flare stars, RS CVns, and Algol binary systems, characterised by strong magnetic activity which drives high energy processes in their atmospheres. Their radio flux density is highly variable and it usually shows two different regimes: quiescent periods, during which a basal flux density of few mJy is observed, and active periods, characterised by a continuous strong flaring which can last for several days (Umana et al. 1995).

Both quiescent and flaring radio emission show spectral and polarisation characteristics consistent with non-thermal radio emission, probably driven by the magnetic activity manifested at other wavelengths. In this scenario, the radio flux is due to gyrosynchrotron emission (Guedel 2009), caused by the interaction of the stellar magnetic field with mildly relativistic particles. A similar mechanism causes the radio emission from pre-main sequence (PMS) stars and Xray binaries. Non-thermal radio emission is also seen from shocks of colliding winds in massive binaries.

Stellar radio flares can occur also as narrow band, rapid, intense, and highly polarised (up to $100 \%$ ) radio bursts, which are particularly common at low frequency $(<1.5 \mathrm{GHz})$. They are believed to be the result of coherent emission mechanisms, requiring a strong magnetic field (which may be variable) and a source of energetic particles. Coherent burst emission has been observed in RS CVns, flare stars, brown dwarfs (BD), and chemically peculiar stars (CPs) (Slee, Wilson, \& Ramsay 2008; Berger 2002; Trigilio et al. 2000). Coherent emission has been detected in just a few tens of stars, because of the limited sensitivity of the available instruments.

Stellar radio emission often exhibits significant circular polarisation (Trigilio et al. 2011; Ravi et al. 2010). While some extragalactic sources do exhibit circular polarisation (e.g. Rayner, Norris, \& Sault 2000), the degree of polarisation is small (typically $\sim 0.01 \%$ ), compared with the levels up to $100 \%$ seen in radio stars. It is therefore important, for the purposes of distinguishing stars from extragalactic objects, that surveys record circular as well as linear polarisation.

Thermal emission (bremsstrahlung emission) is expected from winds associated with WR and OB stars, shells surrounding $\mathrm{PNe}$, and novae and jets from symbiotic stars and class 0 PMS stars (Guedel 2002). In all these cases, radio continuum emission is an important diagnostic to understand the underlying physics. For example, radio continuum observations are the most precise way yet to determine the mass loss of stellar winds. This is particularly important when other diagnostics cannot be used, such as in the case of dustenshrouded objects (Umana et al. 2005).

Since no sensitive survey for radio stars has yet been conducted, little statistical information on stellar radio emission is available. Forecasts of the number of sources detectable with SPARCS surveys rely on radio observations of small samples of stellar sources, usually selected on the basis of observed peculiarities at other wavelengths. Assuming typical radio luminosities for each type of radio star, we conclude that stellar winds and non-thermal radio emission from many active binaries, flares stars, PMS will be easily detected with an rms sensitivity of $10 \mu \mathrm{Jy}^{\text {beam }^{-1}}$ (EMU), while SKA $\left(1 \mu \mathrm{Jy}_{\text {beam }}{ }^{-1} \mathrm{rms}\right)$ will be able to detect a quiescent Sun at $10 \mathrm{pc}$ (see Figure 23).

Until now, targeted observations of well-known radio stars have constituted the best approach to investigate their radio emission mechanisms (e.g. flare development, spectral and polarisation evolution, emission mechanism, etc.). An all-sky survey would significantly enlarge the known stellar radio emitting database, free from selection effects. Results from such a survey would provide new insights into the physics of active stellar systems and plasma processes.

Two areas of stellar radio emission will particularly benefit from SKA and SPARCS surveys. One is the study of 


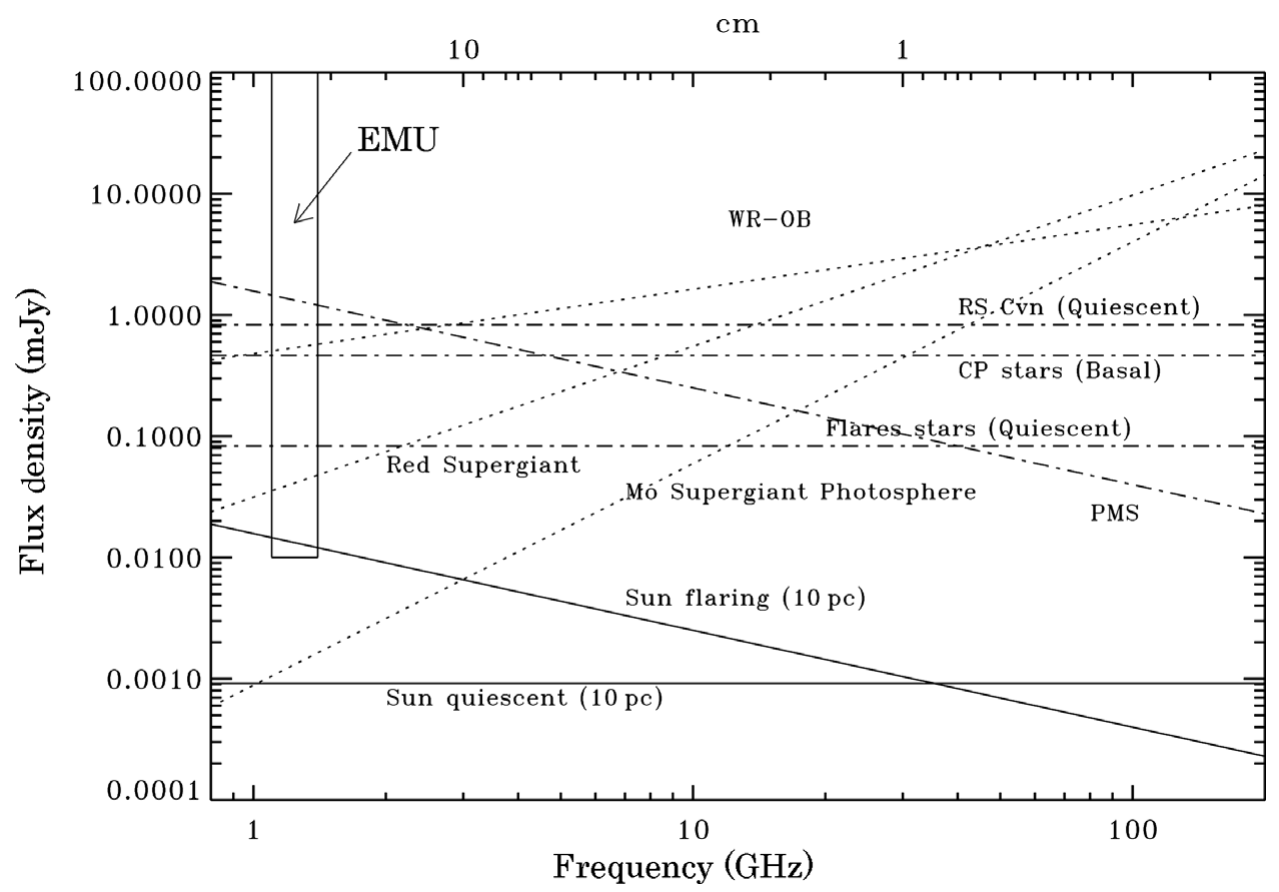

Figure 23. Typical radio spectrum of several classes of radio emitting stars. Fluxes have been derived from the radio luminosity (Seaquist, Krogulec, \& Taylor 1993; Guedel 2002; Umana, Trigilio, \& Catalano 1998; Trigilio et al. 2008; Berger 2006) assuming an appropriate distance for each type of radio star (10 pc for flare stars, $100 \mathrm{pc}$ for active binary systems, $1 \mathrm{kpc}$ for supergiants, $\mathrm{OB}$ and WR, $500 \mathrm{pc}$ for CP stars). The bandwidth and sensitivity of EMU have also been indicated.

gyrosynchrotron stellar flares, where multi-epoch, multifrequency observations will enable the detection of serendipitous flaring activity, allowing the derivation of a typical behaviour (occurrence rate, variation amplitude, etc.) from a statistical study of a larger source population. Moreover, detailed studies of a large number of stellar coronae will be possible, allowing us to understand the nature of energy release in the upper atmospheres of stars of different mass and age and to investigate the correlation between radio and $\mathrm{X}$ ray emission and thus on the Neupert effect (Guedel 2009). The expected sensitivity will also test the presence of superflares on solar-type stars, and search for correlations with an orbiting hot Jupiter (Maehara et al. 2012).

The other important area is related to the study of coherent emission. The EMU plus WODAN surveys will offer the best opportunity yet to determine how common coherent radio emission is from stellar and sub-stellar systems. The detection of coherent emission from a large fraction of surveyed stars will have immense implications for our understanding of both stellar magnetic activity and the dynamo mechanism generating magnetic fields in fully convective stars and brown dwarfs (Hallinan et al. 2008; Ravi et al. 2011). Coherent emission observed in active and BD stars shares several characteristics with that observed in CP stars (Trigilio et al. 2000), since both require a large-scale magnetosphere and are similar to the low frequency coherent radio emission observed from the magnetised planets in our solar system (Trigilio et al. 2011). The well-known topology of CPs magnetic fields, independently derived from optical observations, makes this kind of object an excellent laboratory for stellar magnetosphere studies. Matching of predicted emission with that observed yields parameters of the radio source, such as the surface magnetic field, the number density of the emitting electrons, and the energy spectrum of the electrons. In turn, this provides clues to the acceleration process, the size of the magnetosphere (Alfvén radius) and the inclination of the rotational axis.

If coherent emission is present in many radio active stars, with the same characteristics, it will constitute an excellent diagnostic for star magnetospheres, and a powerful probe of magnetic field topology. In one CP star (CU Vir) the coherent emission is stable on a timescale of years, and has been used to time the rotation of the star, revealing a likely change of its period (Trigilio et al. 2008; Ravi et al. 2010). The discovery of other similar radio lighthouses will enable high-precision studies of the rotation period, and thus angular momentum evolution, in different classes of stars.

\subsection{Unexpected discoveries}

Experience has shown that many great discoveries in astronomy have been made, not by testing a hypothesis, but by observing the sky in an innovative way. The necessary conditions for this to take place are (a) a telescope observing an unexplored part of the observational phase space (frequency, resolution, time domain, area of sky, etc.); (b) an intelligent observer who understands the instrument sufficiently well to distinguish between artefact and discovery; and (c) a prepared 
and enthusiastic mind ready to accommodate and interpret a new discovery.

SKA pathfinder surveys will easily satisfy (a), if only in terms of the numbers of objects surveyed. However, their petabytes of data, and arms-length access, may prevent an observer from satisfying (b) and (c). Although we may hope that someone will eventually stumble across any unexpected phenomena in the data, the impenetrable size of the database implies dark nooks that may never be fully explored. If we rely on serendipity, discoveries may remain undiscovered, for ever.

An alternative is to harness data-mining techniques to help the intelligent observer search for the unexpected. For example, the WTF (Widefield ouTlier Finder) (e.g. Norris et al. 2011b) project will systematically mine the EMU database, searching for the unexpected by discarding objects that already fit known classes of object, using a variety of approaches including

- decision tree analysis

- cluster analysis

- k-furthest neighbour (kFN) by analogy with the kNN technique (P.-C. Zinn et al., in preparation)

- Bayesian analysis.

Identified objects/regions will be either processing artefacts (which are themselves important for data quality control), statistical outliers of known classes of object, or, in a few cases, genuinely new classes of object.

\section{TECHNICAL CHALLENGES}

\subsection{Survey design and quality}

The SKA pathfinder radio continuum surveys may be characterised by the following parameters:

- point-source detection limit,

- angular resolution,

- brightness sensitivity,

- dynamic range,

- frequency coverage,

- polarisation,

- accuracy:

- flux densities,

- positions,

- polarisation,

- image fidelity,

- uniformity in sensitivity.

All of these need to be addressed for these surveys. Ironically, the more ambitious a survey, the more susceptible it will be to small systematic errors. The ambition of the surveys therefore needs to be matched by a corresponding level of effort to understand subtle sources of error and uncertainty.
For example, many radio astronomers have been content to tolerate $10 \%$ uncertainty in their measurements of flux density, while we know that our instruments are, in principle, capable of measuring flux densities to $1 \%$. Reasons for this failure include deconvolution errors, errors in primary beam shape and pointing, failing to correct for missing extended flux, resolution bias, bandwidth smearing, time smearing, CLEAN bias, Eddington bias, etc. To overcome these effects (and probably find new effects which we have not yet met) will doubtless require a significant amount of effort to understand the instrument and its errors, but that effort is justified by the scientific return from these enormous surveys.

It will also be important to present the data to users in ways that are clear and unambiguous, with uniform coverage and easily understood survey parameters. For example, most surveys produce catalogues chosen at a $\sim 5 \sigma$ level of confidence. For some purposes, a less reliable catalogue at a $3 \sigma$ level (for greater numbers of sources) may be useful, while for other studies a threshold of $8 \sigma$ or $10 \sigma$ level (for greater reliability) may be more appropriate (e.g. Raccanelli et al. 2011 assume $10 \sigma)$. While an $8 \sigma$ subset can always be chosen from a $5 \sigma$ sample, it may cause less confusion amongst users for the survey to generate these different levels of catalogue.

It is also important to remember that these surveys will be used for purposes that have not yet been thought of. For example, one of the most exciting results from the NVSS was the measurement of the RM sky (and hence the Galaxy's magnetic field; Taylor et al. 2009). That was not planned at all during the survey, but was subsequently possible because the survey had excellent continuum calibration.

Management of large surveys is likely to be an issue, with previous large radio surveys having had relatively few people with the responsibility for conducting the survey in contrast to the emerging model in which large teams are responsible.

\subsection{The SPARCS reference fields}

The uniformity required from the SPARCS surveys cannot be achieved by simply ensuring that the calibrators are on the same flux scale, although this is an essential first step. For example, the fluxes of strong sources in the first ATLAS data release (Norris et al. 2006; Middelberg et al. 2008b) agreed with those measured by the VLA, while weaker ATCA sources were systematically weaker than weak VLA sources, an effect which turned out to be caused by incorrect assumptions about bandwidth smearing in mosaicked ATCA data (Hales et al. 2012). Other subtle flux-dependent effects doubtless await us, and consistency between our calibrator sources will not be sufficient to detect or fix them. There have even been suggestions of declination-dependent calibration effects (Parra et al. 2010).

During the commissioning of the SPARCS telescopes, particularly those using the relatively untried PAF technologies, it will be important to repeatedly survey well-studied fields containing a grid of sources, pushing the sensitivity and 
dynamic range until all the effects are well understood and can be corrected for.

The measured flux densities of sources in radio surveys are subject to a large number of subtle corrections and bias effects, and even expert radio astronomers do not always agree on these. For example, there is a continuing debate on the measured scatter in the radio source counts at low flux densities (see Figure 3), with some proponents arguing that it is caused by cosmic variance, and others arguing that it is due to processing and calibration differences. In some cases, reprocessing or reobserving (e.g. Condon et al. 2012) has shown that some variations are caused by different processing paths, although cosmic variance may be sufficient in other cases. The problem may be even worse for EMU and WODAN, as both rely on new PAF technology. To extract the maximum science output from these major projects, relying on new PAF technology that has yet to be sufficiently battle tested, requires careful characterisation of individual survey systematics.

To achieve this, we propose three reference fields at declination $\sim+30^{\circ}, 0^{\circ}$, and $-30^{\circ}$ ), which can be observed by all existing and new radio telescopes. Where possible, they have been chosen to overlap with a field that is well studied at other wavelengths, to maximise the science to be obtained from these observations. The two southern fields are in the CDFS-ATLAS field (Norris et al. 2006) and the COSMOS field (Schinnerer et al. 2007). A northern field (15:30, +29:00) has been chosen within the overlap range of ATCA and Westerbork, within the SLOAN survey area, and at least $2.5^{\circ}$ away from any strong ( $>0.5 \mathrm{Jy}$ ) sources. It is planned to observe all three fields, as far and deeply as possible, with all existing survey telescopes (VLA, ATCA, Westerbork, LOFAR, GMRT, etc.) as well as the new SPARCS surveys.

\subsection{Calibration}

Calibration and imaging of SKA pathfinder surveys is likely to present significantly new challenges compared to earlier surveys. For example:

- Direction-dependent calibration is likely to be important not only for low frequencies, such as LOFAR, but also for intermediate frequencies, such as EMU and probably even for VLA and MeerKAT.

- Because all data are acquired in a spectral-line mode, flagging the data for RFI affects the weighting of the data.

- The timescale of gain variations, such as those resulting from pointing errors, is important. For example, wind gusts have a particularly dramatic effect on pointing errors, in part because the errors scale as the square of the wind velocity. The result is quite rapid pointing variations, which may be difficult to track and correct.

- Experience from multi-epoch observations with the WSRT has illustrated the importance of monitoring the calibration information over time. In particular, there can be time variability of the calibration information (e.g., a variable source in the FOV of the calibrator), which could affect the calibration or result in apparent variables.

- The scientific drivers for high dynamic range imaging need to be identified and kept clear. There may not be a need for uniform and high dynamic range across the entire sky.

\subsection{Imaging}

At the excellent sensitivities offered by SKA pathfinder telescopes, imaging and calibration algorithms need to deal simultaneously with issues of

- wide bandwidth (e.g. primary beam corrections and polarisation leakage terms change with frequency),

- wide-field imaging,

- advanced multi-scale deconvolution techniques for imaging extended emission,

- directionally-dependent effects such as instrumental and ionospheric errors,

- the need for high dynamic range and high polarisation purity.

Furthermore, the inherent data volumes are large (typically petabytes) so that computational efficiency and the number of data traversals are important parameters to consider in designing post-processing strategies.

\subsubsection{Existing algorithms}

Two distinct approaches are being pursued in the community to correct for wide-field and direction-dependent effects, which can be classified as (a) projection algorithms and (b) faceting algorithms (Bhatnagar 2009).

Projection algorithms are based on physical modelling of the various direction-dependent (DD) terms of the measurement equation and incorporating them as part of the forward and reverse transforms. These algorithms therefore do not require assumptions about the source brightness distribution. This allows them to take advantage of the FFT algorithm for Fourier transforms, as well as integrate well with advanced deconvolution techniques for scale-sensitive wideband imaging.

Faceting algorithms, on the other hand, are based on data partitioning in such a way that standard direction independent techniques can be applied to the partitions (Nijboer \& Noordam 2007; Mitchell et al. 2008; Smirnov 2011). This approach is phenomenological by design requiring no physical understanding or modelling of the DD term in the measurement equation. They can however suffer from nonoptimal use of the available signal-to-noise ratio (due to data partitioning), curse of dimensionality (too many degrees of freedom) and computational load. To alleviate some of these problems, in addition to the problem of degrees of freedom, 
assumptions about the structure of the brightness distribution typically have to be made.

The W-Projection algorithm (Cornwell, Golap \& Bhatnagar 2008) incorporates the effects of non-coplanar baselines and corrects for its effects during imaging. The A-Projection algorithm (Bhatnagar et al. 2008) similarly accounts for the time, frequency, and polarisation dependence of the antenna primary beams modelling the antenna aperture illumination patterns (or the measured illumination patterns). The combined AW-Projection algorithm therefore can be used at low frequencies where the effects of the W-Term as well as that of antenna primary beams limit the imaging performance. Antenna pointing errors are thought to limit the imaging performance at high frequencies and in mosaic imaging at all frequencies. Using A-Projection for forward and reverse transforms, the Pointing SelfCal algorithm (Bhatnagar, Cornwell, \& Golap 2004) solves for the time-dependent antenna pointing errors. The pointing solutions are then incorporated as part of the A-Projection to correct for the effects of antenna pointing errors.

The Multi-Scale Clean (Cornwell 2008) and Asp-Clean (Bhatnagar \& Cornwell 2004) algorithms are advanced image deconvolution techniques for imaging fields with extended emission. The MS-MFS algorithm (Rau \& Cornwell 2010) for wide-band multi-frequency synthesis not only deals with the deconvolution of extended emission, but also accounts for spatially resolved spectral index variation across the FOV. The combination of AW-Projection and MS-MFS algorithms can therefore be used to account simultaneously for all the dominant wide-field wide-band effects which are expected to limit the imaging performance with SKA pathfinder telescopes.

The algorithmic design of the combined AW-Projection and MS-MFS algorithm is such that it falls in the category of embarrassingly parallel algorithm. To mitigate the fundamentally higher computing load, work is in progress to deploy these combined advanced algorithms on highperformance computing (HPC) platforms, typically consisting of a cluster of computers with multi-core CPUs and high bandwidth interconnect (Bhatnagar, Ye, \& Schiebel 2009).

Since these algorithms also need to iterate over a large volume of data, computing clusters connected to a parallel file system (e.g. the Lustre file system) and using multi-threaded I/O techniques are currently being used. Tests with up to a few Terabytes of data show close to linear scaling in computing with number of computing nodes, although this is subject to the achievable compute-to-I/O ratio. Work is in progress to address the problem of improving this ratio while keeping the resulting memory footprint in reasonable limits. For SKA-sized data sets, iterating over the entire data set might not be feasible. Work is in progress to devise computing schemes and algorithms that may eliminate or at least reduce the number of iterations involving the entire data.
4.4.2 Development of new deconvolution algorithms In the last few years, the theory of compressed sensing (CS; Donoho 2006; Candès, Romberg \& Tao 2006) has been developed by the signal/image processing community, and may be applicable to radio-astronomical image deconvolution.

The theory of CS mostly addresses random sampling, sparse signals, and coarse-grained dictionaries. In radio interferometry, sampling is usually structured, astronomical images are not sparse, and dictionaries must be fine-grained to adequately represent the images. Theoretical CS results therefore do not usually improve estimates of astrophysical sources from interferometric data. However, a key ingredient of the CS framework, sparse representations (Mallat 2008), has demonstrated significant improvements in radioastronomical deconvolution, and has been used as a tool for deconvolution of extended and diffuse sources in the image plane (Wiaux et al. 2009a; Li et al. 2011a; Dabbech, Mary, \& Ferrari 2012) as well as in Faraday depth (Li et al. 2011b).

Sparse representation theory (Fornassier 2010) may be seen as a generalisation of ideas exploited in CLEAN (e.g. Högbom 1974; Wakker \& Schwarz 1988; Cornwell 2008). CLEAN performs an iterative deconvolution by using a dictionary of shifted PSFs to construct a sparse dictionary of point sources. Sparse representation theory aims to construct a dictionary of more complex geometrical features than point sources, addressing three fundamental issues of astronomical image restoration. First, the sparsity of the dictionary reduces the indeterminacy and instability caused by the zeros of the transfer function of an interferometer. Second, the theory allows flexible and sophisticated models that can cope with complex astrophysical sources. Third, it offers computationally efficient optimisation techniques to solve the resulting deconvolution problem.

Sparse representations can take either of two approaches: synthesis and analysis (Elad et al. 2005).

In the synthesis approach, the unknown intensity distribution $\boldsymbol{x}$ (of size $(N, 1)$, say) is assumed to be sparsely synthesisable by a few atoms of a given full rank dictionary $\boldsymbol{S}$ of size $(N, L)$. Hence, we write $\boldsymbol{x}$ as $\boldsymbol{x}=\boldsymbol{S} \pm b \boldsymbol{\gamma}$, where $\pm b \boldsymbol{\gamma}$ (the synthesis coefficients vector) is sparse. In contrast, the analysis approach assumes that $\boldsymbol{x}$ is not correlated with some atoms of an analysis dictionary $\boldsymbol{A}$ of size $(N, L): \boldsymbol{A}^{T} \boldsymbol{x}$ is sparse.

Since real images can often be approximated by a linear combination of a few elementary geometrical features, the synthesis approach is more intuitive and has been the focus of more research. Its design simplicity has also made it popular in image processing applications such as compression, de-noising, and in-painting. However, because a synthesisbased deconvolved image is restricted to a subspace of the synthesis dictionary, the resulting astrophysical images may be too rough to be realistic. On the other hand, since the signal is not built from a small number of atoms, the analysis approach may be more robust to 'false detections'. However, the number of unknowns in the synthesis approach (the number of atoms in the dictionary) may be much larger than in the 
analysis approach. Thus, while analysis-based optimisation strategies may be computationally and qualitatively more efficient for large dictionaries (Starck, Murtagh, \& Fadili 2010), the question of which approach is best remains open (Gribonval \& Schnass 2009, and references therein).

Regardless of the approach chosen, a sparsity-based deconvolution method still requires a dictionary, chosen from a class of images (Mallat 2008). Astronomical wavelet dictionaries are widely used, but sometimes fail to represent asymmetric structures adequately. In such cases, it may be necessary to use other transforms designed for specific classes of objects, such as curvelets, which are optimum for curved, elongated patterns such as planetary rings or galaxy arms, and shapelets, which are often optimum for galaxy morphologies. Modelling complex images may require several dictionaries to be concatenated into a larger dictionary (Chen, Donoho \& Saunders 1998; Gribonval \& Nielsen 2003), although computational issues limit the size of dictionaries, especially for radio synthesis images containing hundreds of thousands of Fourier samples.

Several deconvolution methods that combine these approaches have recently been suggested (e.g. Suksmono 2009; Wiaux et al. 2009a, 2009b; Vannier et al. 2010; Wenger et al. 2010; McEwen \& Wiaux 2011)), including successful simulations of SKA pathfinder observations. Li et al. (2011a) present a classical synthesis approach with an IUWT (Isotropic Undecimated Wavelet Transform) synthesis dictionary, Carillo, McEwen, \& Wiaux (2012) used an analysis approach using a concatenation of wavelet bases, and Dabbech et al. (2012) defined a hybrid analysis-by-synthesis approach.

While the theoretical results of CS have not yet generated tools which can be used routinely for synthesis imaging, they have brought new perspectives from the domain of sparse representations: improved sparsity models and improved optimisation algorithms. The increased research effort on fundamental models for sparse representations (analysis/synthesis/hybrid models) leads to improved models and thus to improved reconstruction. Research in sparse representations is active and growing, and promises valuable future developments in radio-astronomical deconvolution algorithms.

\subsection{Source extraction and measurement}

There are many approaches to source extraction, each with different strengths and weaknesses, so it is important to mount a joint effort to determine how an optimum source finder would work, and what algorithms to implement. In such a joint effort, a reference data set should be subjected to the various algorithms, for optimum comparability.

Building a source finder is likely to be subject to compromise, and current source finders fall well short of what may be theoretically achievable. For example, no source finder currently accounts for variations in the PSF across the image. Development of an 'optimum' source finder may well require collaboration with computer scientists with no astron- omy background to develop signal extraction algorithms. It is likely that no single algorithm will be optimum for all scenarios.

\subsubsection{Compact source extraction}

Two main areas of recent focus have been investigations into the impact of background and noise estimation on the detection of compact sources, and a rigorous comparison of existing source finding software tools. The first of these focuses on source detection, where the background and noise levels in an image are characterised (Huynh et al. 2012).

The second focuses on source characterisation, where islands of pixels are described or fit in some well-defined manner (Hancock et al. 2012). The most common way to describe a source in radio astronomy is as a group of Gaussian components.

The process of background characterisation has been explored by Huynh et al. (2012), who tested the steps of background and noise measurement, and of the choice of a threshold high enough to reject false sources yet not so high that the catalogues are significantly incomplete. This analysis explored the results from testing the background and thresholding algorithms as implemented in the SEXTRACTOR, Duchamp/Selavy, and sFIND tools on simulated data. The result of this analysis shows clearly that it will be crucial to first develop and then implement an automated algorithm for establishing the appropriate scale size on which to estimate the 'local' background and rms noise values for each image, and that this may not be common to all images. This analysis also suggests that the false discovery rate approach (Hopkins et al. 2002) to thresholding may be the most robust, optimising for both completeness and reliability.

The source characterisation stage has been found to have its own distinct issues (Hancock et al. 2012). For a selection of commonly used source finding tools (SFIND, SEXTRACTOR, IMSAD, and SELAVY) there are significant differences in the way that islands of sources are divided into components and subsequently fit. This is exacerbated with examples of sources which are blended or adjacent, especially when there is a large difference in the relative flux densities (even though the fainter component(s) may in their own right be very high signal-to-noise ratio objects). Such behaviour has particular implications for variable objects. If one such source were variable or transient, this behaviour could be missed if a source finding program is not able to correctly decompose the island. If a source finder's ability to decompose an island into components is related to the clipping limit, or background noise, then a source of constant flux could be interpreted as transient or variable due to irregularities in the source characterisation process.

These issues have triggered the development of new algorithms (e.g. Hancock et al. 2012; Hales et al. 2012) that aim to determine the correct number of components within an island, and to generate an initial parameter set for the fitting of multiple Gaussian components. Hancock et al. (2012) use a Laplace transform, to identify regions of negative 
curvature in the pixel intensity profiles, combined with the map defined from the islands of pixels above a threshold, to automate the process of identifying the numbers of components in each island, and their initial position and peak flux density estimates.

Another clear requirement that has developed from these analyses is the need to quantify the false detection rate of sources as a function of signal-to-noise ratio level, and perhaps other image or telescope parameters, in order to assess the likelihood that any detected source is actually real. The impact of even a $0.1 \%$ false detection rate when catalogues of many tens or hundreds of millions of sources are being anticipated is clearly large.

These initial steps have demonstrated that while source finding has been ubiquitous in radio astronomy for many decades, there is still scope for optimising and quantifying the process, and that careful attention will need to be paid to these details in the lead up to the new generation of radio telescopes. There will be many lessons to be learned in order to ensure that source measurement using the SKA, ultimately, can be made as robust as possible.

\subsubsection{Extended source extraction}

Many of the feature recognition algorithms widely applied in fields such as optical character recognition, machine vision, or medical imaging are absent from the repertoire of techniques routinely employed by astrophysicists. However, the increasing complexity and scale of astronomical data are driving an interest in more advanced feature extraction techniques for astronomical contexts. In particular, radio surveys on next-generation telescopes will generate image data containing tens of millions of astronomical sources, many of which will be extended.

Localised kernel transforms, such as the wavelet transform, are often used for astronomical purposes, including diffuse source detection (e.g. detection of diffuse X-ray emission in the COSMOS field; Finoguenov et al. 2006). They are created either by modifying a conventional kernel transforms, or by using exotic basis functions, and are supplemented by a localisation algorithm to allow the target to be located. The success of these methods relies on 'matching' the basis function to the problem at hand. Given the variety of source morphologies in astronomical images, no single transform will adequately detect all sources. Localised kernel transforms work best in cases where a single class of objects can be matched to a suitable set of basis functions, and can also be used to separate diffuse from point source components using curvelet, ridgelet, or á trous transforms (e.g. Figure 24).

Compressive processes rely on the fact that pixels in an image are not independent, so it is possible to encode the patterns in the image rather than the pixel-by-pixel information. This provides a more efficient 'compressed' representation of the information by providing a 'model' of the image. Object detection is then applied to this 'model' rather than to the real data, greatly reducing the complexity of the problem.
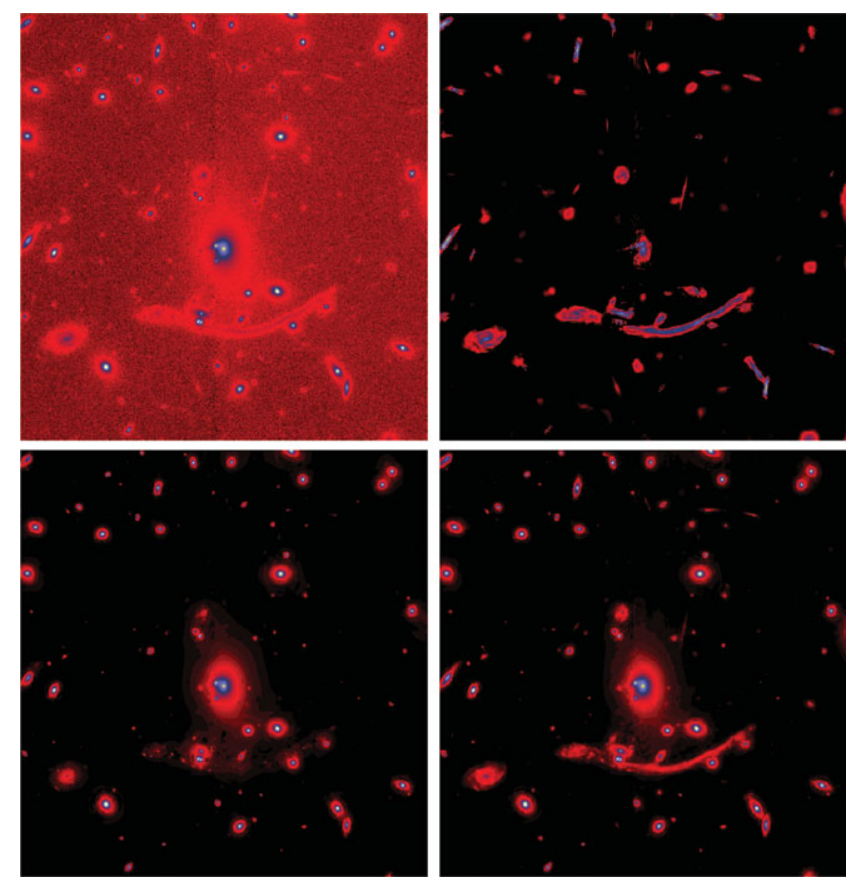

Figure 24. Feature extraction in the HST image of Abell 370 from Starck, Donoho, \& Candés (2003). The top left panel shows the original HST image, top right shows the co-added image from the ridgelet and the curvelet transforms which extract extended features, bottom left shows the reconstruction from the à trous algorithm which responds to point sources, and bottom right shows the addition of the results of all three transforms.

Compression attempts to preserve the information inherent in the original image but does not attempt to abstract geometric or semantic information. Compressive techniques therefore preserve large-scale noise features in addition to diffuse sources. The use of compressive sampling for deconvolution has been discussed in Section 4.4.2.

Template matching is a process where an image is correlated with a target object of known form, and is similar to 'matched filtering'. This technique has been used in radio astronomy for pulsar timing prediction (van Straten 2006; Oslowski et al. 2011), transient source detection (Trott et al. 2012), and to remove radio point source contaminants from diffuse source backgrounds (Pindor et al. 2011). Two disadvantages of template matching are that (a) it is hard to control the required number of filters, resulting in a greater number than for localised kernel transforms, and (b) each template must fit a range of orientation and scaling. Even when the computational complexity of template matching is reduced by combining it with a scale-invariant transform such as the Mellin transform, orientation must still be calculated making this technique very computationally expensive. On the other hand, it has the advantages that (a) the templates do not have to be precise, and cartoon-like models suffice; (b) the method is robust against artefacts; (c) it can be applied in either the image or Fourier domains; and (d) it returns the size, orientation, location type of detected sources. 


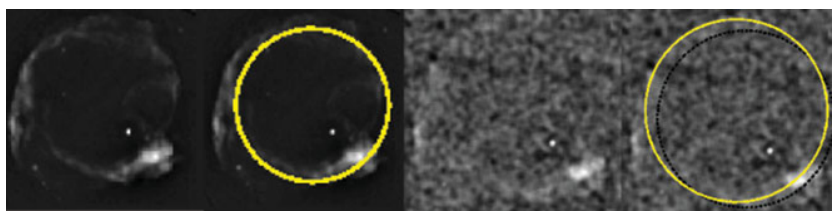

Figure 25. Application of the circle Hough transform to detect radio supernova remnants in the Molonglo Galactic Plane Survey. Left to right: the original image of G315.4-2.3, the location and size of the remnant as found by the CHT, the original image with ten times the Gaussian noise and the response of the $\mathrm{CHT}$ in the noise case (yellow) compared with the original (black), which demonstrates the robustness of CHTs to noise (Hollitt \& Johnston-Hollitt 2012).

Hough transforms can find and characterise geometrical objects such as lines, circles, and ellipses. In general, the Hough transform is equivalent to template matching for some geometric shape, although it inherently incorporates scaling and orientation constraints. Circle or elliptical Hough transforms are particularly relevant to astronomical source detection to determine questions such as the size and location of a circle/ellipse, and have the advantage that the geometrical abstraction limits the number of filters required (e.g. different types of objects contain circular and arc-like features). The circle Hough transform (CHT) can find partial circular objects and is robust to noise. The CHT inputs an image and outputs a 3D array in which two dimensions represent the possible locations for the centres of circles and the third dimension spans the set of possible circle radii. Peaks in this so-called 'Hough space' therefore correspond to circles in the input image. In its simplest form, the circle Hough transform is computationally challenging, with both computational effort and memory consumption scaling as $O\left(n^{3}\right)$ when transforming an $n \times n$ pixel image. Recently, the transform has been recast via a convolution approach which reduces the complexity to $O\left(n^{2}\right) \log n$ (Hollitt 2009, 2012). Although this is not the fastest approach for astronomical source detection, it has been applied as a source detection method for a variety of extended radio sources with arc-like features including supernova remnants, tailed radio galaxies, and radio relics (Hollitt \& Johnston-Hollitt 2009, 2012). For example, Figure 25 shows the detection of a supernova remnant. Hough transforms return semantically useful information such as position, size, orientation, and eccentricity, but will produce somewhat less information than a full template matching process. For example, it cannot distinguish between a supernova remnant and a tailed radio galaxy.

Segmentation methods assume nothing about a source but rather seek to group pixels together based on differences with the background. Three main classes of segmentation methods are used in astrophysics: region growing, edge detection, and waterfalling. The simplest version of a segmentation method is to set a threshold and assume everything above that threshold is a target. In practice, adjoining pixels above the threshold are grouped together as a single target. This approach is usually too simplistic and requires an extension via one of the three subclasses above. Region growing, which includes floodfilling techniques, compares adjacent pixels at the edge of a target and groups them together based on some tolerance in pixel value (e.g. Duchamp and BLOBCAT; Whiting 2012; C. A. Hales et al., in preparation). This technique alone is poor at dealing with disjointed areas, although it may be heuristically augmented to define nearby areas as one object. Another method, edge detection, uses thresholds to find the borders between different 'types' of pixel. This is rarely useful in astronomy because sources have 'soft' edges. Waterfalling involves considering the image as a topographic surface and calculating the intensity gradient, pixels with the lowest gradient denoting watershed lines which are used to bound regions. An advantage of segmentation methods is their speed, making them good for large area surveys. One of the downfalls of segmentation methods is that no semantic information is returned. An additional classifier step is required to be applied to the output of segmentation algorithms to determine semantic properties.

While algorithms for the detection of point sources are well established, automatic detection and characterisation of diffuse sources presents a significant challenge to large radio surveys. While methods such as local kernel transforms, template matching, and Hough transforms have been demonstrated to detect and characterise diffuse sources, they are too computationally expensive to apply to large images. The favoured approach at present is therefore a combination of segmentation algorithms with heuristics to extract diffuse source catalogues. This combined process is not likely to be sufficient for future survey science goals and some combination of techniques, possibly applied on different resolution scales, will be required.

\subsection{Classification and cross-identification}

The large area surveys proposed by the SKA pathfinder projects will return many tens to hundreds of millions of sources, requiring automated techniques both to group components into sources and to identify the counterparts at other wavelengths.

Table 2 lists the current, or planned, large-area multiwavelength surveys that have significant overlap with nextgeneration radio surveys. Based on analysis of current deep multiwavelength fields such as COSMOS and GOODS-N (Scoville et al. 2007; Schinnerer et al. 2007; Morrison et al. 2010; Giavalisco et al. 2004), we estimate that, assuming a limit of rms $\sim 10 \mu \mathrm{Jy}$ beam $^{-1}$ at $1.4 \mathrm{GHz}, \sim 50 \%$ will have counterparts in areas coincident with deep optical surveys (i.e. DES and Pan-STARRS), while $\sim 70 \%$ will have nearIR counterparts in the overlap regions with surveys such as Vista Hemisphere Survey (VHS) and VIKING. However, because most of the deeper surveys only cover small areas, about $50 \%$ of radio sources will have no counterparts at other wavelengths, with this fraction falling to at most $30 \%$ in the $\sim 1000-2000 \mathrm{deg}^{2}$ overlap with the deepest large-area optical-near IR surveys. 
Table 2. Key multiwavelength surveys with which EMU/WODAN data will be cross-identified (restricted to surveys larger than $1000 \mathrm{deg}^{2}$ ) adapted from Norris et al. (2011b). All magnitudes are in AB.

\begin{tabular}{|c|c|c|c|c|c|c|}
\hline $\begin{array}{l}\text { Survey } \\
\text { name }\end{array}$ & $\begin{array}{l}\text { Area } \\
\left(\mathrm{deg}^{2}\right)\end{array}$ & $\begin{array}{l}\text { Wavelength } \\
\text { bands }\end{array}$ & $\begin{array}{l}\text { Limiting } \\
\text { mag. flux }{ }^{a}\end{array}$ & $\begin{array}{l}\text { Detectable } \\
(\%)\end{array}$ & $\begin{array}{l}\text { Matched } \\
(\%)\end{array}$ & $\begin{array}{l}\text { Data } \\
\text { release date }\end{array}$ \\
\hline WISE $^{1}$ & 40000 & $3.4,4.6,12,22 \mu \mathrm{m}$ & $80 \mu \mathrm{Jy}$ & 23 & 23 & 2012 \\
\hline Pan-STARRS ${ }^{2}$ & 30000 & $g, r, i, z, y$ & $r<24.0$ & 54 & 41 & 2020 \\
\hline Wallaby $^{3, \mathrm{~b}}$ & 30000 & $20 \mathrm{~cm}(\mathrm{H} \mathrm{I})$ & $1.6 \mathrm{mJy}^{\mathrm{c}}$ & 1 & 1 & 2013 \\
\hline LSST $^{4}$ & 20000 & $u, g, r, i, z, y$ & $r<27.5$ & 96 & 48 & 2020 \\
\hline Skymapper $^{5}$ & 20000 & $u, v, g, r, i, z$ & $r<22.6$ & 31 & 16 & 2015 \\
\hline VHS $^{6}$ & 20000 & $Y, J, H, K$ & $K<20.5$ & 49 & 25 & 2012 \\
\hline SDSS $^{7}$ & 12000 & $u, g, r, i, z$ & $r<22.2$ & 28 & 8 & DR8 2011 \\
\hline $\mathrm{DES}^{8}$ & 5000 & $g, r, i, z, y$ & $r<25$ & 71 & 9 & 2017 \\
\hline VST-ATLAS $^{9}$ & 4500 & $u, g, r, i, z$ & $r<22.3$ & 30 & 4 & $2012 ?$ \\
\hline Viking $^{10}$ & 1500 & $Y, J, H, K$ & $K<21.5$ & 68 & 3 & 2012 \\
\hline Pan-STARRS Deep 2 & 1200 & $0.5-0.8, g, r, i, z, y$ & $g<27.0$ & 57 & 2 & 2020 \\
\hline
\end{tabular}

Notes. The 'detectable' column is the fraction of 1.4-GHz EMU/WODAN sources that are in principle detectable by the multiwavelength survey to its $5 \sigma$ limit. The 'matched' column is the fraction of $1.4 \mathrm{GHz}$ sources which are both detectable and in the area of sky covered by the multiwavelength survey. The sensitivity shown for the WISE survey is for the $3.5 \mu \mathrm{m}$ band.

${ }^{\text {a Denotes }} 5 \sigma$ point source detection. However, in many cases, a priori positional information will enable $3 \sigma$ data to be used, resulting in a higher detection rate.

${ }^{\mathrm{b}}$ Being an $\mathrm{H}$ I survey, WALLABY will measure redshifts for all detected galaxies out to $z=0.26$.

${ }^{\mathrm{c}}$ Per $4 \mathrm{~km} \mathrm{~s}^{-1}$ channel achieved in 8-h integration.

References. ${ }^{1}$ Wright et al. (2010); ${ }^{2}$ Kaiser et al. (2010); ${ }^{3}$ B. S. Koribalski et al. (in preparation); ${ }^{4}$ Ivezic et al. (2008); ${ }^{5}$ Keller et al. (2007); ${ }^{6}$ http://www.ast.cam.ac.uk/research/instrumentation.surveys.and.projects/vista; ${ }^{7} \mathrm{Abazajian}$ et al. (2009); ${ }^{8}$ The Dark Energy Survey Collaboration (2005); ${ }^{9}$ Shanks (2005); ${ }^{10} \mathrm{http}: / /$ www.eso.org/sci/observing/policies/ PublicSurveys/sciencePublicSurveys.html

Padovani (2011) has estimated cross-identification rates for the SKA down to nJy radio flux densities. Figure 26 plots $R_{\text {mag }}$ versus $1.4-\mathrm{GHz}$ radio flux density and shows the expected $R_{\text {mag }}$ for various classes of sources that will be found in the radio surveys.

Figure 26 shows that, down to $50 \mu \mathrm{Jy}, \approx 50 \%$ of SFGs should be detected by the SDSS (northern hemisphere) and SkyMapper (southern hemisphere), while most AGNs will be fainter. Pan-STARSS and the LSST should detect most of the radio sources down to this flux density but on longer timescales (e.g. by $\sim 2028$ in the case of the LSST, assuming a start of operations in 2018). Surveys reaching $1 \mu \mathrm{Jy}$ (e.g. those from the VLA and MIGHTEE) will obviously have much fainter counterparts and might require JWST and the Extremely Large Telescopes (ELTs) for the optical identification of most of their sources, which however will be covering a relatively small FOV (up to a few $\operatorname{arcmin}^{2}$ ). X-ray surveys are intrinsically much less sensitive than optical/IR surveys and will detect only a very small fraction of the AGNs detected by radio surveys (Padovani 2011).

The requirements for spectroscopic redshifts are obviously even more difficult. For example, long exposures $(\sim 10 \mathrm{~h})$ with $8 / 10 \mathrm{~m}$ telescopes can secure redshifts in the case of strong emission lines down to $R_{\text {mag }} \approx 26$. Large-area spectroscopic surveys, however, cannot reach these magnitudes. For example, current magnitude limits are $r \leq 17.8$ (galaxies) and $i \leq 19.1$ (quasars) for the SDSS and $b_{J} \leq 20.85$ for the $2 \mathrm{dF}$ QSO redshift survey.

The source density of potential matches will probably be $>1$ per radio beam, so simply taking the nearest neighbour to the radio source in other catalogues is not a feasible approach. Furthermore, radio sources will typically have more than one component per source, so a simple nearest-neighbour algorithm will attempt to match sources to the lobes of an FRII galaxy as well as to the core. Clearly, more sophisticated matching algorithms must be considered

A significant number of techniques exist to facilitate the matching of catalogues. Techniques such as the $p$-statistic (Downes et al. 1986) and the likelihood ratio (Sutherland \& Saunders 1992) make use of the number density of background (and true counterpart) sources to 'weigh' potential counterparts within a given search radius, but cannot take into account the likelihood that several radio components may correspond to one optical component. More recently, Budavári \& Szalay (2008) outlined a Bayesian method to determine the most likely match. This method, while similar to the likelihood ratio, is distinct in that it does not consider the source density, and hence is less biased towards faint counterparts (although potentially less reliable for very high source densities). A Bayesian approach (Budavári 2011) also allows a number of other constraints to be built into the process for selecting the most likely candidate. This can in principle even extend to including the knowledge that the core of an FRII is associated with a host galaxy while the lobes are not. This balance between reliability of matches, and completeness as a function of flux, will be key in deciding which algorithm is appropriate for the scientific exploitation of radio surveys.

Finally, it is likely that any automated algorithm will fail on complex radio sources, which may include those which are most scientifically interesting. For these cases, a 'Citizen 


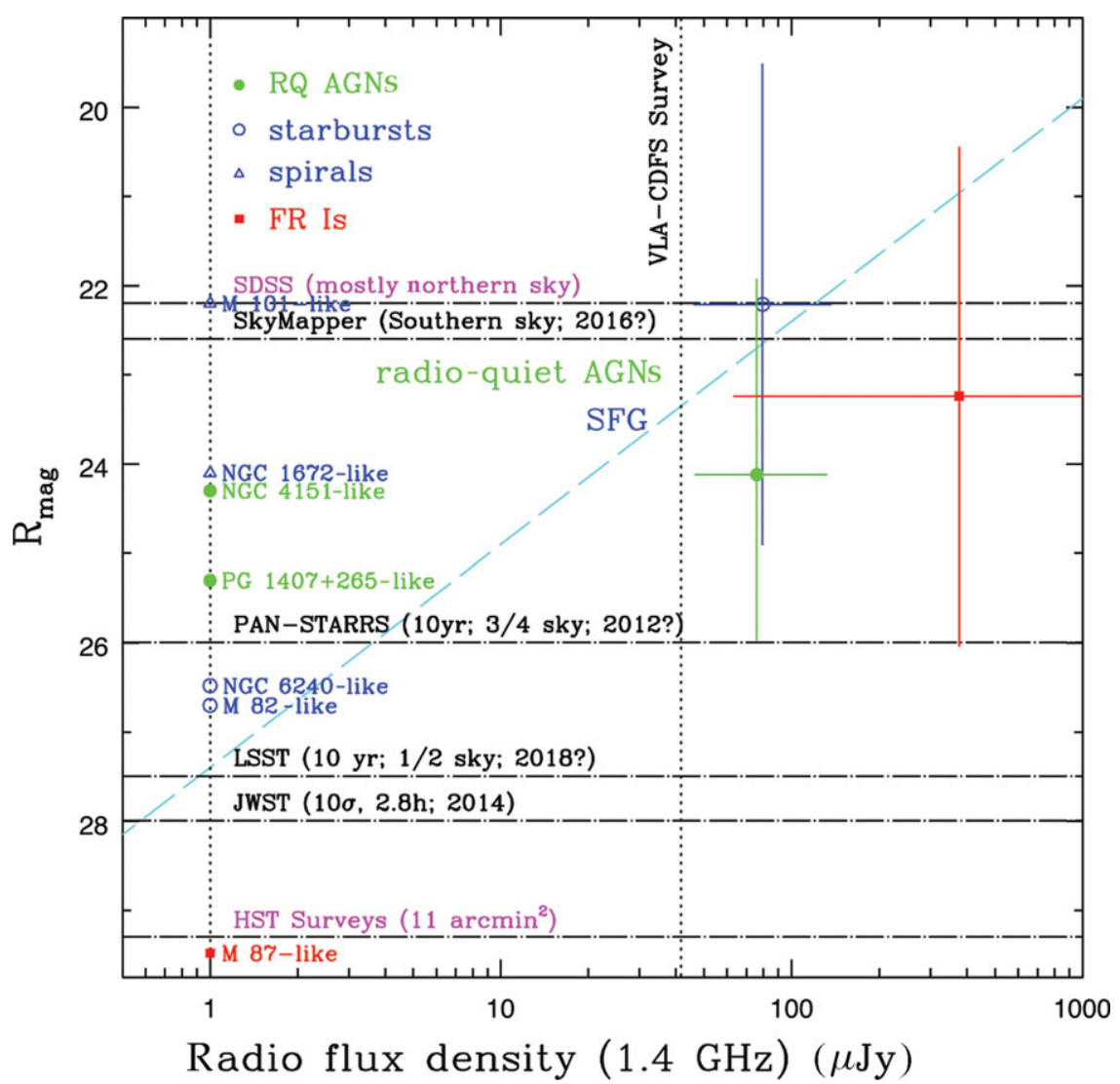

Figure 26. $R_{\mathrm{mag}}$ versus the 1.4-GHz radio flux density for faint radio sources. The diagonal dashed line indicates the maximum value for SFG and the approximate dividing line between radio-loud and radio-quiet AGNs, with SFGs and radio-quiet AGNs expected to populate the top left part of the diagram. The typical $R$ magnitudes of the three classes at $S_{1.4}=1 \mu \mathrm{Jy}$ are also shown, with SFG split into starbursts and spirals. Finally, the mean radio and $R_{\text {mag }}$ values for sources from the VLA-CDFS sample, with error bars indicating the standard deviation, are also marked. The horizontal dot-dashed lines indicate the approximate point-source limits of planned and existing surveys.

Science' approach is being developed to enlist the help of thousands of enthusiastic amateurs, in collaboration with the Galaxy Zoo group (Lintott et al. 2008) to examine each of the millions of complex sources by eye. The 'Radio Zoo' project expects to release its first prototype in early 2013.

\subsubsection{The likelihood ratio technique for radio source cross-identification}

The likelihood ratio (LR) technique (e.g. Richter 1975; Sutherland \& Saunders 1992; Cilegi et al. 2003) can be used to identify optical/near-infrared counterparts with sources selected from low resolution survey data. Smith et al. (2011) discuss a recent application of this Bayesian technique to identify 2423 optical counterparts to 6621 sources from the Herschel-Astrophysical TeraHertz Large Area Survey (Herschel-ATLAS; Eales et al. 2010), selected at $250 \mu \mathrm{m}$.

The LR technique relies on the brightness and astrometric properties of the input catalogues in general, and each individual source in particular, to determine the ratio of the probability that two objects are related, to the probability that they are unrelated. After using an empirical method to account for the fraction of sources which are not detected in the optical/near-infrared catalogue, we can determine the reliability of each association, with values ranging from 0 (not related) to 1 (a match).

The likely positional uncertainties, coupled with the probable optical/near-infrared catalogues that will be available for identifying counterparts to the new catalogues that these surveys will generate, suggest that, apart from the problem of radio sources with multiple components, the problem will be very similar to that addressed using the LR technique in the Herschel-ATLAS.

McAlpine et al. (2012) have begun detailed investigations based on existing deep radio survey data and new nearinfrared data from the VISTA VIDEO survey to quantify how effective the LR technique will be for these purposes, and how it will be affected by using data of different sensitivity and spatial resolution.

\subsubsection{Bayesian approaches}

Budavári \& Szalay (2008) propose Bayesian hypothesis testing for cross-identification. Given a set of detections, one 
can ask directly whether the measured directions are consistent with a common single object. This hypothesis is tested against its complement using the Bayes factor, which is the likelihood ratio

$$
B=\frac{L_{\text {same }}}{L_{\text {not }}},
$$

where the likelihoods are calculated as a sum over all possible model parameters, e.g., the true position of the source. Assuming that the astrometric uncertainty is well approximated with a normal distribution, the derivation is analytic. For example, in the case of two detections of point sources with high-precision circular uncertainties and an all-sky prior on the true position

$$
B=\frac{2}{\sigma_{1}^{2}+\sigma_{2}^{2}} \exp \left\{-\frac{\psi^{2}}{2\left(\sigma_{1}^{2}+\sigma_{2}^{2}\right)}\right\},
$$

where $\psi$ is the angular separation of the detections and $\sigma_{i}$ are the uncertainties, all in radians. When this dimensionless quantity is larger than 1 , the data support the association. The higher the value, the greater is the evidence. The probability is analytically given in terms of $B$ and the prior of the hypothesis, which is a function of the source densities of the input data sets. The approach is applicable to different kinds of data, such as fluxes or polarisation. The corresponding Bayes factors are computed independently and simply multiplied together. The method's firm statistical foundation along with its explicit dependence on the geometry and physical modelling provides a clean framework for catalogue associations at all wavelengths and straightforward extensions. Such examples include the association of stars with unknown proper motions (Kerekes et al. 2010) or transient events (T. Budavári, in preparation).

\subsection{Measuring redshifts}

To obtain the full value of a radio survey, it is essential to have multiwavelength identification and redshifts. However, only about $1 \%$ of radio sources from these surveys will have spectroscopic redshifts (Section 4.6), and so it is necessary to rely on photometric redshifts or even statistical redshifts.

The most popular way to compute photometric redshifts is via fitting of templates of galaxies to the measured multi-band photometry of target galaxies. Clearly, the accuracy of the result depends not only on the library of templates, but also on the number of photometric points available and their associated errors. Other techniques, such as k-nearest neighbour $(\mathrm{kNN})$ implementations, support vector regression models, self-organised maps, Gaussian processing, neural-networks, and rainbow forest, do not make any assumptions about the SED of the galaxies or the type and amount of extinction. Instead, they build on a simple colour-redshift relation using a training sample of sources with reliable spectroscopic redshifts. These methods are very efficient and fast whenever the training sample is representative of the entire population, and become less reliable outside the redshift range for which they were trained.

About $20 \%$ of EMU sources will have photometric data from the six optical bands of the Skymapper survey, the two near-infrared bands of VHS, and the four mid-infrared bands of WISE (see Table 2). Typically, with these available data sets, an error in redshift of $\sim 0.03$ can be reached for normal galaxies up to redshift $z \sim 1.5$ using either standard (SED template fitting) or empirical (machine learning) techniques.

About 20\% of identified sources will be classified as AGNs, based on one or more of the following criteria: (a) radio morphology, (b) radio polarisation, (c) X-ray detection, (d) a radio spectral index very different from $\alpha \sim-0.7$, and (e) a radio/IR ratio very different from the canonical SF value. About $5 \%$ of the sources will be QSOs, detectable by the next X-ray all-sky survey, eROSITA (Cappelluti et al. 2011; Merloni et al. 2012), and for these sources a special treatment must be adopted (Salvato et al. 2009, 2011).

About $80 \%$ of the EMU sources will have an optical counterpart that is fainter than $r=22 \operatorname{mag}_{A B}$ and for these sources the ancillary multiwavelength data set will be coarse and non homogeneous. Finally, the training sample with spectroscopic redshift available at high redshift may not be as complete as at $z<1$, hampering the empirical photometric redshift techniques.

With the final goal of providing the most reliable photometric redshift for the larger number of sources possible, the EMU redshift working group is testing all the methods mentioned above, trying to understand the advantages and disadvantages of each of them, and thus applying the most suitable, or a combination, of the methods, depending on (a) the available depth of the multiwavelength data and (b) on the redshift range available for the training sample. We started experimenting on COSMOS field (Scoville et al. 2007) representative of a pencil beam survey $\left(2 \mathrm{deg}^{2}\right)$ with a very deep and homogeneous multiwavelength data set, but for which the training spectroscopic sample is limited to lowredshift radio sources, and with a strong component of X-raydetected sources. Other fields which we will be testing are the larger area ATLAS (Norris et al. 2006; Middelberg et al. 2008b), which has deep but non-homogeneous ancillary data, and GAMA $\left(310 \mathrm{deg}^{2}\right)$, for which the training spectroscopic sample is rich but representing only the nearby universe $(r<$ $20 \mathrm{mag}$ ) and with limited availability of ancillary data.

In addition to the optical, near, and mid-infrared data, we will make use of the radio properties of the sources. While for the empirical methods the radio flux will be used as an additional band, for the SED-fitting technique, we will use the radio properties as a prior for limiting the redshift range or the templates used for the possible solutions.

The 'statistical redshift' concept, introduced by Norris (2011), recognises that a probabilistic estimate of redshift for a large sample can be useful, even if many of them are incorrect, provided that the level of incorrectness and incompleteness can be accurately calibrated. Even a non-detection 
can carry useful information, and radio data themselves can add significantly to the choice of SED template. For example,

- high-redshift radio galaxies can be identified from their strong radio emission coupled with a NIR non-detection (Willott et al. 2003; Norris et al. 2011a);

- a steep radio spectral index increases the probability of a high redshift (De Breuck et al. 2002);

- the angular size of a particular galaxy class can be loosely correlated with redshift (Wardle \& Miley 1974);

- polarised radio sources are nearly always AGNs (C. A. Hales et al., in preparation), while unpolarised sources are mainly SF galaxies. Therefore, for the EMU/WODAN surveys, polarised sources have a mean $z \sim 1.8$, while unpolarised sources have a mean $z \sim 1.1$.

\subsection{Data issues}

\subsubsection{Stacking and data-intensive research}

Stacking is used to explore the properties of a class of objects which are below the detection limit of a survey. For example, the radio flux of RQQs can be measured by averaging the measured radio flux at the position of each RQQ in a radio survey, even though each individual RQQ has a flux well below the detection limit.

More generally, the process of stacking involves combining (typically by taking a censored mean or median) the data at the position where such objects are expected in the survey. The noise tends to cancel, while any low level of flux density in the sources adds, resulting in a detection threshold very much lower than that of the unstacked survey. Stacking at radio wavelengths has been used very successfully (e.g. Boyle et al. 2007; White 2007; Ivison et al. 2007, 2010; Dunne et al. 2009; Messias et al. 2010; Bourne et al. 2011) on highresolution data for the purpose of studying faint populations which are below the detection threshold of the radio image, and has proved to be a powerful tool for studying SFRs, AGN activity, radio-infrared correlation, and measuring the fraction of the extragalactic background contributed by various source populations. Jack-knifing techniques are currently being explored to provide more information about the flux density distribution of stacked sources than is available from a simple mean or median (Rees et al., in preparation).

The unprecedented area-depth product of SPARCS surveys makes them very suitable for stacking. For example, stacking a sample of a million optically selected galaxies in the EMU data will result in a noise level of $\sim 10 \mathrm{nJy}$. Because of the wide area of the EMU, WODAN, and LOFAR surveys, even rare classes of sources can be stacked, and it should also be possible to create stacked images of extended sources, such as clusters. However, the extent to which such deep stacking will be successful will depend on the extent to which imaging artefacts are cancelled by stacking. Stacking is also not without its hazards, and can be biased by a number of effects such as failure to account for the PSF, particularly in the presence of confusion and in highly clustered fields (Bourne et al. 2011; Greve et al. 2010; Penner et al. 2011; Chary, Cooray, \& Sullivan 2008; Béthermin et al. 2010).

Other examples of data-intensive research include:

- Identification of sources which do not fit into known categories of radio source, and so are likely to be artefacts or exciting new classes of source, as discussed in Section 3.9.

- Extraction of low surface brightness radio emission to detect the WHIM synchrotron emission from cosmic filaments or sheets, by cross-correlating with optical galaxies selected from other surveys.

- Cross-correlation of low-redshift galaxies with highredshift galaxies, or the CMB, to test cosmology and fundamental physics as discussed in Section 3.6.

\subsubsection{Survey versus pointed data}

Since surveys compete for observing time with conventional 'pointed' observations, it is useful to consider their role compared with projects doing detailed science on particular sources or fields. Clearly both modes are valuable and complementary. Surveys provide source lists for follow-up observations of particular sources, as well as finding and characterising calibrators for other radio observations. However, the huge volume of data from the new radio surveys will trigger a shift in the way astronomers 'observe'. The ingenuity currently applied to devising a key experiment, and crafting a successful proposal, will be applied to devising novel ways of mining the data. To some extent, this is already happening, and about three times as many citations are delivered by results from the HST/STScI archive than from the papers written by PIs. The surveys discussed here will also have an enormous legacy value, since they will be hard to improve on until the technology makes a further major step forward.

\section{CONCLUSION}

The SKA Pathfinder Radio Continuum Survey working group (SPARCS) has been established to coordinate the science and technical developments associated with continuum surveys being planned or undertaken with the SKA pathfinder telescopes (APERTIF, ASKAP, VLA, $e$-MERLIN, e-EVN, LOFAR, MeerKAT). This review paper was triggered by the first SPARCS workshop, and is an attempt not only to share the knowledge residing in the individual pathfinder projects but also to establish a baseline of knowledge from which all projects can move on, and which can be used to optimise the development of the SKA. A primary outcome of the first SPARCS workshop was to rethink aspects of the way we are constructing the SKA pathfinder telescopes and designing the surveys. For example, it became clear that the impact of these surveys on cosmology and fundamental physics may dwarf the key science goals we have been working on for the 
last few years. That places demands on source-measurement accuracy and uniformity which in turn impacts on calibration and imaging accuracy.

Key demonstrable outcomes of the workshop include this review paper, and the establishment of a series of reference fields in both hemispheres which are now starting to be observed with major existing telescopes (VLA, ATCA, Westerbork) and will be observed with the new pathfinder telescopes (LOFAR, Aperitif, ASKAP, MeerKAT) to ensure consistency and uniformity across the surveys.

In this review paper, we have explored the current state of science to be tackled with the SPARCS surveys, and identified the most pressing challenges. We conclude that:

- The SKA pathfinder telescopes and upgrades, and their planned continuum surveys, have complementary capabilities which will lead to ground breaking science. Particularly exciting are (a) the ability of EMU and WODAN to cover the entire sky with high sensitivity and resolution, detecting about 100 million galaxies; (b) the ability of LOFAR to make complementary observations of much of that region at low frequency; (c) the ability of POSSUM and BEOWULF to obtain detailed information about the magnetic sky over the same region; and (d) the ability of $e$-MERLIN, VLA, e-EVN, and MIGHTEE to study selected areas and objects with very high sensitivity and resolution.

- Together, these surveys are likely to make major inroads on some of the pressing questions about the origin and evolution of galaxies, including distinguishing AGN and SF components of galaxies, tracing the evolution of the luminosity function for both, and exploring the causes and signatures of hot-mode and cold-mode accretion.

- Amongst the sources detected by the surveys will be very high redshift galaxies, possibly the highest discovered, but identifying them, and measuring their redshift will be challenging.

- The SPARCS surveys are likely to detect tens to hundreds of thousands of new clusters, and will also map the extended halo and relic emission from a fraction of these. Comparison with X-ray observations is likely to yield new insights into the physics of clusters and the growth of structure in the Universe.

- The measurement of polarisation and RMs is an important part of the surveys, and is likely to yield insight not only into the physics of the radio galaxies but into the origin of cosmic magnetism. There is a good chance that the intergalactic magnetic field will be detected and traced by these surveys.

- Even without redshifts, several cosmological probes can use SPARCS survey data to measure cosmological parameters and test models of gravity, with significantly more precision than current analyses. The combination of WODAN and EMU is likely to yield the best measurement yet of the ISW effect. The most powerfully constraining probe of dark energy parameters turns out to be the source count correlations, while ISW provides the most stringent constraints on modified gravity theories. If photometric redshifts can be obtained for a significant subset of the radio sources, then these cosmological tests become even more powerful.

- Variable and transient sources are currently poorly studied, and the SPARCS surveys are likely to change this field significantly. In addition to constraining models of e.g. black hole accretion, these studies are also likely to result in unexpected discoveries.

- While most SPARCS surveys are focussed on extragalactic science, they will also produce the best highresolution data yet on the Galaxy, detecting essentially every SNR and UCHII region in the Galaxy, and vastly increasing the number of known radio stars. It is essential that this scientifically important aspect of the SPARCS surveys is not neglected in our drive to answer the key questions of extragalactic astrophysics.

- Because the SPARCS surveys are opening up a large area of unexplored parameter space, it is likely that they will make unexpected discoveries. It is important not to leave this process to chance, but to recognise it as a legitimate goal of large surveys, and to plan processes and software accordingly, to mine the deluge of data that these surveys will produce.

To achieve these exciting scientific goals, many technical challenges must be addressed by the survey instruments. Given the limited resources of the global radio-astronomical community, it is essential that we pool our skills and knowledge. We do not have sufficient resources to enjoy the luxury of re-inventing wheels. We face significant challenges in calibration, imaging, source extraction and measurement, classification and cross-identification, redshift determination, stacking, and data-intensive research. As the SPARCS instruments extend the observational parameters, we will face further unexpected challenges in calibration, imaging, and interpretation. If we are to realise the full scientific potential of these expensive instruments, it is essential that we devote enough resources and careful study to understanding the instrumental effects and how they will affect the data. The prime role of SPARCS is to facilitate the process of doing so by ensuring we share resources and expertise across the projects.

\section{ACKNOWLEDGMENTS}

We are indebted to the Lorentz Center in Leiden for hosting and funding the workshop in 2011 February, which gave rise to this paper. Parts of this research were supported by the Australian Research Council Centre of Excellence for All-sky Astrophysics (CAASTRO), through project number CE110001020. Part of the research described in this paper was carried out at the Jet Propulsion Laboratory, California Institute of Technology, under a 
contract with the National Aeronautics and Space Administration. JA gratefully acknowledges support from the Science and Technology Foundation (FCT, Portugal) through the research grants PTDC/FIS/100170/2008, PTDC/CTEAST/105287/2008, and PEst-OE/FIS/UI2751/2011. CF and $\mathrm{AD}$ acknowledge financial support by the Agence Nationale de la Recherche through grant ANR-09-JCJC-0001-01. AD acknowledges financial support from the joint $\mathrm{PhD}$ program of Observatoire de la Côte d'Azur and Conseil régional Provence-Alpes-Côte d'Azur.

\section{REFERENCES}

Abazajian, K. N., et al. 2009, ApJS, 182, 543

Afonso, J., Mobasher, B., Chan, B., \& Cram, L. 2001, ApJ, 559, L101

Afonso, J., Mobasher, B., Koekemoer, A., Norris, R. P., \& Cram, L. 2006, AJ, 131, 1216

Afonso, J., et al. 2009, in Proceedings of Panoramic Radio Astronomy: Wide-field 1-2 GHz Research on Galaxy Evolution, ed. G. Heald \& P. Serra. Published online at http://pos.sissa.it/cgibin/reader/conf.cgi?confid $=89$, id.64

Afonso, J., et al. 2011, ApJ, 743, 122

Albrecht, A., et al. 2006, arXiv:astro-ph/0609591

Alexandroff, R., et al. 2012, MNRAS, 423, 1325

Ananthakrishnan, S., \& Pramesh Rao, A. 2001, in 2001 Asia-Pacific Radio Science Conference AP-RASC '01, 237

Archibald, E. N., Dunlop, J. S., Hughes, D. H., Rawlings, S., Eales, S. A., \& Ivison, R. J. 2001, MNRAS, 323, 417

Arshakian, T. G., Beck, R., Krause, M., \& Sokoloff, D. 2009, A\&A, 494, 21

Bacchi, M., Feretti, L., Giovannini, G., \& Govoni, F. 2003, A\&A, 400, 465

Bagchi, J., Durret, F., Neto, G. B. L., \& Paul, S. 2006, Sci, 314, 791

Baldwin, J. A., Phillips, M. M., \& Terlevich, R. 1981, PASP, 93, 5

Banerjee, R., \& Jedamzik, K. 2003, PhRvL, 91, 251301

Bannister, K. W., Murphy, T., Gaensler, B. M., Hunstead, R. W., \& Chatterjee, S. 2011, MNRAS, 418, 2813

Battye, R., et al. 2011, http://www.e-merlin.ac.uk/legacy/projects/ superclass.html

Beck, R. 2009, Ap\&SS, 320, 77

Beck, R. 2011, AIP Conf. Ser., 1381, 117

Beck, R., Frick, P., Stepanov, R., \& Sokoloff, D. 2012, A\&A, 543A, 113

Beck, R., \& Gaensler, B. M. 2004, NewAR, 48, 1289

Beck, R., et al. 2010, http://www.astron.nl/radioobservatory/APERTIF-eoi-abstracts-and-contact-information

Becker, R. H., Helfand, D. J., White, R. L., \& Proctor, D. D. 2010, AJ, 140, 157

Becker, R. H., White, R. L., \& Helfand, D. J. 1995, ApJ, 450, 559

Bell, E. F. 2003, ApJ, 586, 794

Bell, M. E., et al. 2011, MNRAS, 415, 2

Berger, E. 2002, ApJ, 572, 503

Berger, E. 2006, ApJ, 648, 629

Bernardi, G., et al. 2009, A\&A, 500, 965

Bernardi, G., et al. 2012, ApJ, submitted

Best, P. N., Arts, J. N., Röttgering, H. J. A., Rengelink, R., Brookes, M. H., \& Wall, J. 2003, MNRAS, 346, 627

Best, P. N., \& Heckman, T. M. 2012, MNRAS, 421, 1569
Best, P. N., Kaiser, C. R., Heckman, T. M., \& Kauffmann, G. 2006, MNRAS, 368, L67

Best, P. N., von der Linden, A., Kauffmann, G., Heckman, T. M., \& Kaiser, C. R. 2007, MNRAS, 379, 894

Best, P. N., Kauffmann, G., Heckman, T. M., Brinchmann, J., Charlot, S., Ivezić, Ž., \& White, S. D. M. 2005, MNRAS, 362, 25

Béthermin, M., Dole, H., Beelen, A., \& Aussel, H. 2010, A\&A, 512, A78

Bhatnagar, S. 2009, ASPC, 407, 375

Bhatnagar, S., \& Corwnell, T. J. 2004, A\&A, 426, 747

Bhatnagar, S., Cornwell, T. J., \& Golap, K. 2004, EVLA Memo 84

Bhatnagar, S., Cornwell, T. J., Golap, K., \& Uson, J. M. 2008 A\&A, 487, 419

Bhatnagar, S., Ye, H., \& Schiebel, D. 2009 EVLA Memo 133

Biggs, A. D., \& Ivison, R. J. 2006, MNRAS, 371, 963

Biggs, A. D., \& Ivison, R. J. 2008, MNRAS, 385, 893

Biggs, A. D. Younger J. D., \& Ivison, R. J. 2010, MNRAS, 408, 342

Blake, C., Bacon, D., Fluke, C., Kitching, T., Miller, L., Power, C., \& Wilman, R. 2007, SKADS Virtual Telescope Proposal, Available at http://www.skads-eu.org/p/svt/svt_blake.pdf

Blake, C., \& Wall, J. 2002, MNRAS, 329, L37

Blanton, E. L., Gregg, M. D., Helfand, D. J., Becker, R. H., \& Leighly, K. M. 2001, AJ, 121, 2915

Blanton, E. L., Gregg, M. D., Helfand, D. J., Becker, R. H., \& White, R. L. 2000, ApJ, 531, 118

Blanton, E. L., et al. 2003, AJ, 125, 1635

Bock, D. C.-J., Large, M. I., \& Sadler, E. M. 1999, AJ, 117, 1578

Bonafede, A., Feretti, L., Murgia, M., Govoni, F., Giovannini, G., Dallacasa, D., Dolag, K., \& Taylor, G. B., 2010, A\&A, 513, A30

Bonafede, A., Govoni, F., Feretti, L., Murgia, M., Giovannini, G., Brüggen, M. 2011, A\&A, 530, A24

Bondi, M., et al. 2007, A\&A, 463, 519

Bornancini, C. G., O’Mill, A. L., Gurovich, S., \& Lambas, D. G. 2010, MNRAS, 406, 197

Bourne, N., Dunne, L., Ivison, R. J., Maddox, S. J., Dickinson, M., \& Frayer, D. T. 2011, MNRAS, 410, 1155

Bouwens, R. J., Illingworth, G. D., Franx, M., \& Ford, H. 2008, ApJ, 686, 230

Bower, R. G., Benson, A. J., Malbon, R., Helly, J. C., Frenk, C. S., Baugh, C. M., Cole, S., \& Lacey, C. G. 2006, MNRAS, 370, 645

Bower, G. C., \& Saul, D. 2011, ApJ, 728, L14

Bower, G. C., Saul, D., Bloom, J. S., Bolatto, A., Filippenko, A. V., Foley, R. J., Perley, D. 2007, ApJ, 666, 346

Bower, G. C., et al. 2010, ApJ, 725, 1792

Bowman, J. D., et al. 2012, 2012arXiv1212.5151B

Boyle, B. J., Cornwell, T. J., Middelberg, E., Norris, R. P., Appleton, P. N., \& Smail, I. 2007, MNRAS, 376, 1182

Braun, R., Heald, G., \& Beck, R. 2010, A\&A, 514, A42

Brentjens, M. A. 2011, A\&A, 526, A9

Brentjens, M. A., \& de Bruyn, A. G. 2005, A\&A, 441, 1217

Brown, M. L., \& Battye, R. A. 2011a, MNRAS, 410, 2057

Brown, M. L., \& Battye, R. A. 2011b, ApJ, 735, 23

Brown, S., Duesterhoeft, J., \& Rudnick, L. 2011, ApJ, 727, L25

Brunetti, G., \& Blasi, P. 2005, MNRAS, 363, 1173

Brunetti, G., Cassano, R., Dolag, K., \& Setti, G. 2009, A\&A 507, 661

Brunetti, G., Lazarian, A. 2007, MNRAS, 378, 245

Brunetti, G., Lazarian, A. 2011, MNRAS, 410, 127 
Brunetti, G., Setti, G., Feretti, L., \& Giovannini, G. 2001, MNRAS, 320,365

Brunetti, G., Venturi, T., Dallacasa, D., Cassano, R., Dolag, K., Giacintucci, S., \& Setti, G. 2007, ApJ, 670, L5

Brunetti, G., et al. 2008, Natur, 455, 944

Bryant, J. J., Johnston, H. M., Broderick, J. W., Hunstead, R. W., De Breuck, C., \& Gaensler, B. M. 2009, MNRAS, 395, 1099

Budavári, T. 2011, ApJ, 736, 155

Budavári, T., \& Szalay, A. S. 2008, ApJ, 679, 301

Bunton, J. D., \& Hay, S. G. 2010, in International Conference on Electromagnetics in Advanced Applications (ICEAA), http:// ieeexplore.ieee.org/xpls/abs_all.jsp?arnumber $=5651120$

Burkhart, B., Lazarian, A., \& Gaensler, B. M. 2012, ApJ, 749, 145

Burn, B. J. 1966, MNRAS, 133, 67

Camera, S., Santos, M. G., Bacon, D. J., Jarvis, M. J., McAlpine, K., Norris, R. P., Raccanelli, A., \& Röttgering, H. 2012, MNRAS, 427, 2079

Cameron, A. D., Keith, M., Hobbs, G., Norris, R. P., Mao, M. Y., \& Middelberg, E. 2011, MNRAS, 415, 845

Candès, E. J., Romberg, J., \& Tao, T. 2006, IEEE Trans. Inf. Theory, 52,489

Cappelluti, N., et al. 2011, MSAIS, 17, 159

Caputi, K. I., et al. 2007, ApJ, 660, 97

Carilli, C. L., Ivison, R. J., \& Frail, D. A. 2003, ApJ, 590, 192

Carilli, C., \& Rawlings, S. 2004, NewAR, 48, 979

Carrillo, R. E., McEwen, J. D., \& Wiaux, Y. 2012, MNRAS, 426, 1223

Cassano, R., \& Brunetti, G. 2005, MNRAS, 357, 1313

Cassano, R., Brunetti, G., \& Setti, G. 2006, MNRAS, 369, 1577

Cassano, R., Brunetti, G., Röttgering, H. J. A., \& Brüggen, M. 2010b, A\&A, 509, 68

Cassano, R., Ettori, S., Giacintucci, S., Brunetti, G., Markevitch, M., Venturi, T., \& Gitti, M. 2010a, ApJ, 721, L82

Cassano, R., Brunetti, G., Venturi, T., Setti, G., Dallacasa, D., Giacintucci, S., \& Bardelli, S. 2008, A\&A, 480, 687

Cassano, R., Brunetti, G., Norris, R. P., Röttgering, H. J. A., Johnston-Hollitt, M., \& Trasatti, M. 2012, A\&A, 548, 100

Cavagnolo, K. W., McNamara, B. R., Nulsen, P. E. J., Carilli, C. L., Jones, C., Bîrzan, L. 2010, ApJ, 720, 1066

Chambers, K. C., Miley, G. K., van Breugel, W. J. M., \& Huang, J.-S. 1996, ApJS, 106, 215

Chang, T., Refregier, A., \& Helfand, D. J. 2004, ApJ, 617, 794

Chary, R.-R., Cooray, A., \& Sullivan, I. 2008, ApJ, 681, 53

Chary, R., \& Elbaz, D. 2001, ApJ, 556, 562

Chatterjee, S., Murphy, T., \& VAST Collaboration 2010, BAAS, 42, \#470.12

Chen, S. S., Donoho, D. L., \& Saunders, M. A. 1998, SIAM J. Sci. Comput., 20, 33

Churchwell, E. 2002, ARA\&A, 40, 27

Ciliegi, P., Zamorani, G., Hasinger, G., Lehmann, I., Szokoly, G., \& Wilson, G. 2003, A\&A, 398, 901

Cirasuolo, M., Celotti, A., Magliocchetti, M., \& Danese, L. 2003, MNRAS, 346, 447

Clarke, T. E., Kronberg, P. P., \& Bohringer, H. 2001, ApJ, 547, L111

Clewley, L., \& Jarvis, M. J. 2004, MNRAS, 352, 909

Condon, J. J. 1974, ApJ, 188, 279

Condon, J. J. 1992, ARA\&A, 30, 575

Condon, J. J., \& Backer, D. C. 1975, ApJ, 197, 31

Condon, J. J., Cotton, W. D., Greisen, E. W., Yin, Q. F., Perley, R. A., Taylor, G. B., \& Broderick, J. J. 1998, AJ, 115, 1693

Condon, J. J., \& Kaplan, D. L. 1998, ApJS, 117, 361
Condon, J. J., Kaplan, D. L., \& Terzian, Y. 1999, ApJS, 123, 219

Condon, J. J., Ledden, J. E., Odell, S. L., \& Dennison, B. 1979, AJ, 84,1

Condon, J. J., et al. 2012, ApJ, 758, 23

Cornwell, T. J. 2008, IEEE J. Sel. Top. Signal Process., 2, 793

Cornwell, T. J., Golap, K., \& Bhatnagar, S. 2008 IEEE J. Sel. Top. Signal Process, 2, 647

Cornwell, T. J., et al. 2011, ASKAP Memo ASKAP-SW-0020

Crittenden, R., \& Turok, N. 1996, PhRvL, 76, 575

Croft, S., Bower, G. C., Keating, G., Law, C., Whysong, D., Williams, P. K. G., \& Wright, M. 2011, ApJ, 731, 34

Croft, S., et al. 2010, ApJ, 719, 45

Croton, D. J., et al. 2006, MNRAS, 365, 11

Dabbech, A., Mary, D., \& Ferrari, C., 2012, IEEE Trans. Acoust. Speech Signal Process., 3665

De Breuck, C., van Breugel, W., Röttgering, H., \& Miley, G. 2000, A\&AS, 143, 303

De Breuck, C., van Breugel, W., Stanford, S. A., Röttgering, H., Miley, G., \& Stern, D. 2002, AJ, 123, 637

De Breuck, C., Downes, D., Neri, R., van Breugel, W., Reuland, M., Omont, A., \& Ivison, R. 2005, A\&A, 430, 1

de Oliveira-Costa, A., Kogut, A., Devlin, M. J., Netterfield, C. B., Page, L. A., \& Wollack, E. J. 1997, ApJ, 482, L17

De Zotti, G., Massardi, M., Negrello, M., \& Wall, J. 2010, A\&AR, 18,1

Deboer, D. R., et al. 2009, IEEE Proc., 97, 1507

Dehghan, S., Johnston-Hollitt, M., Mao, M., Norris, R. P., Miller, N. A., \& Huynh, M. 2011, JApA, 32, 491

Deller, A. T., et al. 2011, PASP, 123, 275

Dennison, B., Broderick, J. J., Ledden, J. E., Odell, S. L., \& Condon, J. J. 1981, AJ, 86, 1604

Dennison, B., \& Condon, J. J. 1981, ApJ, 246, 91

Dewdney, P. E., Hall, P. J., Schilizzi, R. T., \& Lazio, T. J. L. W. 2009, Proc. IEEE, 97, 1482

Dolag, K., Kachelriess, M., Ostapchenko, S., \& Tomàs, R. 2011, ApJ, 727, L4

Dolag, K., Bykov, A. M., \& Diaferio, A. 2008, SSRv, 134, 311

Donoho, D. L. 2006, IEEE Trans. Inf. Theory, 52, 1289

Dotti, M., Sesana, A., \& Decarli, R. 2012, AdAst, 940568

Downes, A. J. B., Peacock, J. A., Savage, A., \& Carrie, D. R. 1986, MNRAS, 218, 31

Draine, B. T., \& Lazarian, A. 1998, ApJ, 508, 157

Dunlop, J. S., \& Peacock, J. A. 1990, MNRAS, 247, 19

Dunlop, J. S., Hughes, D. H., Rawlings, S., Eales, S. A., \& Ward, M. J. 1994, Natur, 370, 347

Dunne, L., et al. 2009, MNRAS, 394, 3

Eales, S., et al. 2010, PASP, 122, 499

Elad, M., Starck, J. L., Querre, P., \& Donoho, D. 2005, Appl. Comput. Harmon. Anal., 19, 340

Elbaz, D., et al. 2007, A\&A, 468, 33

Enßlin, T. A., \& Röttgering, H. 2002, A\&A, 396, 83

Erickson, W. C. 1957, ApJ, 126, 480

Fabian, A. C., et al. 2003, MNRAS, 344, L43

Fabian, A. C., et al. 2006, MNRAS, 366, 417

Falcke, H., Körding, E., \& Nagar, N. M. 2004, NewAR, 48, 1157

Fanaroff, B. L., \& Riley, J. M. 1974, MNRAS, 167, 31

Fanidakis, N., Baugh, C. M., Benson, A. J., Bower, R. G., Cole, S., Done, C., \& Frenk, C. S. 2011, MNRAS, 410, 53

Fender, R., Wijers, R., \& Stappers, B. 2008 LOFAR Transients Key Science Project t., 2008, (arXiv:0805.4349) 
Feretti, L., Bacchi, M., Slee, O. B., Giovannini, G., Govoni, F., Andernach, H., \& Tsarevsky, G. 2006, MNRAS, 368, 544

Feretti, L., Fusco-Femiano, R., Giovannini, G., \& Govoni, F. 2001, A\&A, 373, 106

Feretti, L., \& Giovannini, G. 1996, IAUS, 175, 333

Feretti, L., \& Giovannini, G. 2008, LNP, 740, 143

Feretti, L., Giovannini, G., Govoni, F., \& Murgia, M. 2012, A\&AR, 20,54

Feretti, L., Dallacasa, D., Govoni, F., Giovannini, G., Taylor, G. B., \& Klein, U. 1999, A\&A, 344, 472

Fernandes, C. A. C., et al. 2011, MNRAS, 411, 1909

Ferrari, C., Govoni, F., Schindler, S., Bykov, A. M., \& Rephaeli, Y. 2008, SSRv, 134, 93

Feulner, G., Gabasch, A., Salvato, M., Drory, N., Hopp, U., \& Bender, R. 2005, ApJ, 633, L9

Finoguenov, A., David, D. S., Zimmer, M., \& Mulchaey, J. S. 2006, ApJ, 646, 143

Fixsen, D. J., et al. 2011, ApJ, 734, 5

Fomalont, E., \& Reid, M. 2004, NewAR, 48, 1473

Fornassier, M. 2010, Theoretical Foundations and Numerical Methods for Sparse Recovery (1st edn.; Berlin: De Gruyter)

Fornengo, N., Lineros, R., Regis, M., \& Taoso, M. 2011, PhRvL, 107,261302

Foster, T., Kothes, R., Sun, X. H., Reich, W., \& Han, J. L. 2006 , A\&A, 454, 517

Frail, D. A., Kulkarni, S. R., Berger, E., \& Wieringa, M. H. 2003 , AJ, 125, 2299

Frail, D. A., Kulkarni, S. R., Ofek, E. O., Bower, G. C., \& Nakar, E. 2012, ApJ, 747, 70

Frey, S., Paragi, Z., Gurvits, L. I., Cseh, D., \& Gabányi, K. É. 2010, A\&A, 524, 83

Frey, S., Paragi, Z., Gurvits, L. I., Gabányi, K. É., \& Cseh, D. 2011, A\&A, 531, 5

Gaensler, B. M., \& Hunstead, R. W. 2000, PASA, 17, 72

Gaensler, B. M., Landecker, T. L., Taylor, A. R. \& POSSUM Collaboration, 2010, BAAS, 42, 515

Gaensler, B. M., et al. 2011, Natur, 478, 214

Garn, T., \& Alexander, P. 2008, MNRAS, 391, 1000

Garrett, M. A., Wrobel, J. M., \& Morganti, R. 2005, ApJ, 619, 105

Garrington, S. T., et al. 2004, Proc. SPIE, 5489, 332

Georgakakis, A., Mobasher, B., Cram, L., Hopkins, A., Lidman, C., \& Rowan-Robinson, M. 1999, MNRAS, 306, 708

Gilli, R., Comastri, A., \& Hasinger, G. 2007, A\&A, 463, 79

Giavalisco, M., et al. 2004, ApJ, 600, L93

Giovannini, G., \& Feretti, L. 2002, Astrophys. Space Sci. Libr., 272, 197

Giroletti, M., et al. 2011, A\&A, 528, L11

Gobat, R., et al. 2011, A\&A, 526, A133

Godfrey, L., et al. 2012, PASA, 29, 42

Gomez, P. L., et al. 2003, ApJ, 584, 210

Govoni, F., Feretti, L., Giovannini, G., Böhringer, H., Reiprich, T. H., \& Murgia, M. 2001, A\&A, 376, 803

Govoni, F., Murgia, M., Feretti, L., Giovannini, G., Dolag, K., \& Taylor, G. B. 2006, A\&A, 460, 425

Green, D. A. 2009, BASI, 37, 45

Greve, T. R., et al. 2010, ApJ, 719, 483

Gribonval, R., \& Nielsen, M. 2003, IEEE Trans. Inf. Theory, 49, 3320

Gribonval, R., \& Schnass, K. 2009, arXiv:0904.4774

Grießmeier, J.-M., Zarka, P., \& Spreeuw, H. 2007, A\&A, 475, 359

Gruppioni, C., et al. 1999, MNRAS, 305, 297
Guedel, M. 2002, ARA\&A, 40, 217

Guedel, M. 2009, LNP, 778, 269

Gunawardhana, M. L. P., et al. 2011, MNRAS, 415, 1647

Gurvits, L. I. 2004, NewAR, 48, 1211

Hales, C. A., Murphy, T., Curran, J. R., Middelberg, E., Gaensler, B. M., \& Norris, R. P. 2012, MNRAS, 425, 979

Hallinan, G., Antonova, A., Doyle, J. G., Bourke, S., Lane, C., \& Golden, A. 2008, ApJ, 684, 644

Hancock, P., Murphy, T., Gaensler, B., Hopkins, A. M., \& Curran, J. 2012, MNRAS, 422, 1812

Hardcastle, M. J., Evans, D. A., \& Croston, J. H. 2007, MNRAS, 376,1849

Harvey-Smith, L., Madsen, G. J., \& Gaensler, B. M. 2011, ApJ, 736,83

Harvey-Smith, L., Gaensler, B. M., Kothes, R., Townsend, R., Heald, G. H., Ng, C.-Y., \& Green, A. J. 2010, ApJ, 712, 1157

Hasinger, G., Miyaji, T., \& Schmidt, M. 2005, A\&A, 441, 417

Haverkorn, M., Brown, J. C., Gaensler, B. M., \& McClureGriffiths, N. M. 2008, ApJ, 680, 362

Haverkorn, M., Katgert, P., \& de Bruyn, A. G. 2003a, A\&A, 403, 1031

Haverkorn, M., Katgert, P., \& de Bruyn, A. G. 2003b, A\&A, 404, 233

Heald, G. 2009, IAUS, 259, 591

Heald, G., Braun, R., \& Edmonds, R. 2009, A\&A, 503, 409

Helfand, D. J., Becker, R. H., White, R. L., Fallon, A., \& Tuttle, S. 2006, AJ, 131, 2525

Helou, G., Soifer, B. T., \& Rowan-Robinson, M. 1985, ApJ, 298, L7

Högbom, J. A. 1974, A\&AS, 15, 417

Hollitt, C. P. 2009, in 24th Int. Conf. on Image and Vision Computing, New Zealand, 373

Hollitt, C. P. 2012, Mach. Vis. Appl., in press

Hollitt, C., \& Johnston-Hollitt, M. 2009, in Proc. of the 16th Electronics Conf., New Zealand, 113

Hollitt, C. P., \& Johnston-Hollitt, M. 2012, PASA, 29, 309

Hopkins, A. M., \& Beacom, J. F. 2006, ApJ, 651, 142

Hopkins, A. M., et al. 2002, AJ, 123, 1086

Hopkins, A. M., et al. 2003, AJ, 125, 465

Hughes, D. H., Dunlop, J. S., \& Rawlings, S. 1997, MNRAS, 289, 766

Huynh, M. T., Hopkins, A. M., Norris, R. P., Hancock, P., Murphy, T., Jurek, R., \& Whiting, M. 2012, PASA, 29, 229

Huynh, M. T., Norris, R. P., \& Middelberg, M. 2010, ApJ, 710, 698

Huynh, M. T., Jackson, C. A., Norris, R. P., \& Prandoni, I. 2005, AJ, 130, 1373

Ibar, E., Ivison, R. J., Best, P. N., Coppin, K., Pope, A., Smail, I., \& Dunlop, J. S. 2010, MNRAS, 401, L53

Ibar, E., Ivison, R. J., Biggs, A. D., Lal, D. V., Best, P. N., \& Green, D. A. 2009, MNRAS, 397, 281

Ivezic, Z., Tyson, J. A., Allsman, R., Andrew, J., Angel, R., \& for the LSST Collaboration 2008, arXiv:0805.2366

Ivison, R. J. 1995, MNRAS, 275, L33

Ivison, R. J., et al. 1998, ApJ, 494, 211

Ivison, R. J., et al. 2007, ApJ, 660, L77

Ivison, R. J., et al. 2010, MNRAS, 402, 245

Jansson, R., Farrar, G. R., Waelkens, A. H., \& Enßlin, T. A. 2009, JCAP, 7, 21

Jarvis, M. J., Rawlings, S., Eales, S., Blundell, K. M., Bunker, A. J., Croft, S., McLure, R. J., \& Willott, C. J. 2001a, MNRAS, 326, 1585 
Jarvis, M. J., Rawlings, S., Willott, C. J., Blundell, K. M., Eales, S., \& Lacy, M. 2001b, MNRAS, 327, 907

Jarvis, M. J., Teimourian, H., Simpson, C., Smith, D. J. B., Rawlings, S., \& Bonfield, D. 2009, MNRAS, 398, L83

Johnston, S., et al. 2007, PASA, 24, 174

Johnston, S., et al. 2008, ExA, 22, 151

Johnston-Hollitt, M. 2003, PhD thesis, Uni. Adelaide

Johnston-Hollitt, M., \& Ekers, R. D. 2004, arXiv:astro-ph/0411045

Jonas, J. L. 2009, IEEE Proc., 97, 1522

Juneau, S., et al. 2005, ApJ, 619, L135

Kaiser, N., et al. 2010, Proc. SPIE, 7733, 0E

Keller, S. C., et al. 2007, PASA, 24, 1

Kempner, J. C., Blanton, E. L., Clarke, T. E., Enßlin, T. A., Johnston-Hollitt, M., \& Rudnick, L. 2004, in "The Riddle of Cooling Flows in Galaxies and Clusters of Galaxies", ed. T. Reiprich, J. Kempner, \& N. Soker. http://www.astro.virginia. edu/coolflow/

Ker, L. M., Best, P. N., Rigby, E. E., Röttgering, H. J. A., \& Gendre, M. A. 2012, MNRAS, 420, 2644

Kerekes, G., Budavári, T., Csabai, I., Connolly, A. J., \& Szalay, A. S. 2010, ApJ, 719, 59

Kewley, L. J., Heisler, C. A., Dopita, M. A., Sutherland, R., Norris, R. P., Reynolds, J., \& Lumsden, S, 2000, ApJ, 530, 704

King, A. R., Pringle, J. E., \& Hofmann, J. A. 2008, MNRAS, 385, 1621

Kistler, M. D., Yüksel, H., Beacom, J. F., Hopkins, A. M., \& Wyithe, J. S. B. 2009, ApJ, 705, L104

Klamer, I. J., Ekers, R. D., Sadler, E. M., Weiss, A., Hunstead, R. W., \& De Breuck, C. 2005, ApJ, 621, L1

Klamer, I. J., Ekers, R. D., Bryant, J. J., Hunstead, R. W., Sadler, E. M., \& De Breuck, C. 2006, MNRAS, 371, 852

Kodama, T., et al. 2007, MNRAS, 377, 1717

Kogut, A., Banday, A. J., Bennett, C. L., Gorski, K. M., Hinshaw, G., \& Reach, W. T. 1996, ApJ, 460, 1

Kolatt, T. 1998, ApJ, 495, 564

Kothes, R., \& Brown, J.-A. 2009, IAUS, 259, 75

Kothes, R., Fedotov, K., Foster, T. J., \& Uyanıker, B. 2006, A\&A, 457, 1081

Krause, M., Alexander, P., Bolton, R., Geisbüsch, J., Green, D. A., \& Riley, J. 2009, MNRAS, 400, 646

Krolik, J. H., \& Chen, W. 1991, AJ, 102, 1659

Kronberg, P. P. 2004, JKoAS, 37, 501

Kronberg, P. P., Bernet, M. L., Miniati, F., Lilly, S. J., Short, M. B., \& Higdon, D. M. 2008, ApJ, 676, 70

Kukula, M. J., Dunlop, J. S., Hughes, D. H., \& Rawlings, S. 1998, MNRAS, 297, 366

Kurtz, S. 2005, IAUS, 227, 111

Lacy, M., et al. 2004, ApJS, 154, 166

Landecker, T. L., et al. 2010, A\&A, 520, A80

Lazio, T. J. W., et al. 2010, AJ, 140, 1995

Le Floc'h, E., et al. 2005, ApJ, 632, 169

Leitch, E. M., Readhead, A. C. S., Pearson, T. J., \& Myers, S. T. 1997, ApJ, 486, L23

Lewis, I., et al. 2002, MNRAS, 334, 673

Li, F., Brown, S., Cornwell, T.J., \& de Hoog, F. 2011, A\& A, 531, 126

Li, F., Cornwell, T. J., \& de Hoog, F. 2011, A\&A, 528, 31

Linder, E. V. 2003, PhRvL, 90, 091301

Lintott, C. J., et al. 2008, MNRAS, 389, 1179

Lonsdale, C. J., et al. 2009, IEEE Proc., 97, 1497

Lutz, D., et al. 2010, ApJ, 712, 1287
Maehara, H., et al. 2012, Natur, 485, 478

Magliocchetti, M., Maddox, S. J., Wall, J. V., Benn, C. R., \& Cotter, G. 2000, MNRAS, 318, 1047

Magnelli, B., et al. 2010, A\&A, 518, L28

Magorrian, J., et al. 1998 , AJ, 115, 2285

Mallat, S., 2008, A Wavelet Tour of Signal Processing: The Sparse Way (3rd edn.; Academic Press: San Diego)

Manchester, R. N., Hobbs, G. B., Teoh, A., \& Hobbs, M. 2005, AJ, 129, 1993

Mao, M. Y., Huynh, M. T., Norris, R. P., Dickinson, M., Frayer, D., Helou, G., \& Monkiewicz, J. A. 2011a, ApJ, 731, 79

Mao, M. Y., Sharp, R., Saikia, D. J., Norris, R. P., JohnstonHollitt, M., Middelberg, E., \& Lovell, J. E. J. 2011b, JApA, 32, 585

Mao, M. Y., Sharp, R., Saikia, D. J., Norris, R. P., JohnstonHollitt, M., \& Lovell, J. E. J. 2010a, MNRAS, 406, 2578

Mao, S. A., Gaensler, B. M., Haverkorn, M., Zweibel, E. G., Madsen, G. J., McClure-Griffiths, N. M., Shukurov, A., \& Kronberg, P. P. 2010b, ApJ, 714, 1170

Mao, M., et al. 2012, MNRAS 426, 3334

Martínez-Sansigre, A., \& Rawlings, S. 2011, MNRAS, 414, 1937

Martinez-Sansigre, A., et al. 2006, MNRAS, 370, 1479

Martinez, M. A., et al. 2010 AJ, 139, 1199

Mauch, T., \& Sadler, E. M. 2007, MNRAS, 375, 931

Mauduit, J.-C., et al. 2012, PASP, 124, 714

McAlpine, K., \& Jarvis, M. J. 2011, MNRAS, 413, 1054

McAlpine, K., Smith, D. J. B., Jarvis, M. J., Bonfield, D. G., \& Fleuren, S. 2012, MNRAS, 423, 132

McClure-Griffiths, N. M., Madsen, G. J., Gaensler, B. M., McConnell, D., \& Schnitzeler, D. H. F. M. 2010, ApJ, 725, 275

McEwen, J. D., \& Wiaux, Y. 2011, MNRAS, 413, 1318

McNamara, B. R., \& Nulsen, P. E. J. 2007, ARA\&A, 45, 117

Medvedev, M. V., Silva, L. O., Fiore, M., Fonseca, R. A., \& Mori, W. B. 2004, JKoAS, 37, 533

Merloni, A., \& Heinz, S. 2007, MNRAS, 381, 589

Merloni, A., et al. 2012, arXiv:1209.3114

Messias, H., Afonso, J., Hopkins, A., Mobasher, B., Dominici, T., \& Alexander, D. M. 2010, ApJ, 719, 790

Middelberg, E., Norris, R. P., Tingay, S., Mao, M. Y., Phillips, C. J., \& Hotan, A. W. 2008a, A\&A, 491, 435

Middelberg, E., Norris, R. P., Hales, C. A., Seymour, N., JohnstonHollitt, M., Huynh, M. T., Lenc, E., \& Mao, M. Y., 2011a, A\&A, 526, A8

Middelberg, E., et al. 2008b, AJ, 135, 1276

Middelberg, E., et al. 2011b, A\&A, 526, A74

Middelberg, E., et al. 2012, A\&A, in press (2012arXiv1212.4605M)

Mignano, A., Prandoni, I., Gergorini, L., Parma, P., de Ruiter, H. R., Wieringa, M. H., Vettolani, G., \& Ekers, R. D. 2008, A\&A, 477, 459f

Miley, G., \& De Breuck, C. 2008, A\&ARv, 15, 67

Miller, N. A., Fomalont, E. B., Kellermann, K. I., Mainieri, V., Norman, C., Padovani, P., Rosati, P., \& Tozzi, P. 2008, ApJS, 179,114

Miller, C. J., Nichol, R. C., Gómez, P. L., Hopkins, A. M., \& Bernardi, M. 2003, ApJ, 597, 142

Miller, N. A., \& Owen, F. N. 2001, AJ, 121, 1903

Mitchell, K. J., \& Condon, J. J. 1985, AJ, 90, 1957

Mitchell, D. A., Greenhill, L. J., Wayth, R. B., Sault, R. J., Lonsdale, C. J., Cappallo, R. J., Morales, M. F., \& Ord, S. M. 2008, IEEE J. Sel. Top. Signal Process., 2, 707 
Mo, H. J., \& White, S. D. M. 1996, MNRAS, 282, 347

Mobasher, B., et al. 2009, ApJ, 690, 1074

Morgan, J. S., Mantovani, F., Deller, A. T., Brisken, W., Alef, W., Middelberg, E., Nanni, M., \& Tingay, S. 2011, A\&A, 526, A140

Morrison, G. E., Owen, F. N., Dickinson, M., Ivison, R. J., \& Ibar, E. 2010, ApJS, 188, 178

Murgia, M., Govoni, F., Feretti, L., Giovannini, G., Dallacasa, D., Fanti, R., Taylor, G. B., \& Dolag, K. 2004, A\&A, 424, 429

Murphy, E. J. 2009, ApJ, 706, 482

Murphy, E. J., Chary, R.-R., Dickinson, M., Pope, A., \& Lin, L. 2011, ApJ, 732, 126

Murphy, E. J., et al. 2006, ApJ, 638, 157

Murphy, E. J., et al. 2010, ApJ, 709, L108

Murphy, T., et al. 2012, PASA, in press (2012arXiv1207.1528M)

Napier, P. J. 2006, ASPC, 356, 65

Napier, P. J., et al. 1994, IEEE Proc., 82, 658

Nijboer, R. J., \& Noordam, J. E. 2007, Astronomical Data Analysis Software and Systems XVI, 376, 237

Norris, R. P. 2011, JApA, 32, 599

Norris, R. P., Lenc, E., Roy, A. L., \& Spoon, H. 2012, MNRAS, 422, 1453

Norris, R. P., Middelberg, E., \& Boyle, B. J. 2008, in the Modern Radio Universe, PoS(MRU)050

Norris, R. P., Tingay, S., Phillips, C., Middelberg, E., Deller, A., \& Appleton, P. N. 2007, MNRAS, 378, 1434

Norris, R. P., et al. 2006, AJ, 132, 2409

Norris, R. P., et al. 2011a, ApJ, 736, 55

Norris, R. P., et al. 2011b, PASA, 28, 215

Nota, T., \& Katgert, P. 2010, A\&A, 513, A65

Noutsos, A., Johnston, S., Kramer, M., \& Karastergiou, A. 2008, MNRAS, 386, 1881

Oosterloo, T., Verheijen, M., van Cappellen, W, Bakker, L., Heald, G., \& Ivashina, M., 2009, in "Proceedings of Wide Field Astronomy \& Technology for the Square Kilometre Array (SKADS 2009). Published online at http:// pos.sissa.it/cgibin/reader/conf.cgi? confid=132, id.70"

Oslowski, S., van Straten, W., Hobbs, G. B., Bailes, M., \& Demorest, P. 2011, MNRAS, 418, 1258

Overzier, R. A., Röttgering, H. J. A., Rengelink, R. B., \& Wilman, R. J. 2003, A\&A, 405, 53

Owen, F. N., \& Morison, G. E. 2008, AJ, 136, 1889

Owen, F. N., Morrison, G. E., Klimek, M. D., \& Greisen, E. W. 2009, AJ, 137, 4846

Padovani, P. 2011, MNRAS, 411, 1547

Padovani, P., Mainieri, V., Tozzi, P., Kellermann, K. I., Fomalont, E. B., Miller, N., Rosati, P., \& Shaver, P. 2009, ApJ, 694, 235

Padovani, P., Miller, N., Kellermann, K. I., Mainieri, V., Rosati, P., \& Tozzi, P. 2011, ApJ, 740, 20

Papadopoulos, P. P., Röttgering, H. J. A., van der Werf, P. P., Guilloteau, S., Omont, A., van Breugel, W. J. M., \& Tilanus, R. P. J. 2000, ApJ, 528, 626

Parra, R., Conway, J. E., Aalto, S., Appleton, P. N., Norris, R. P., Pihllström, Y. M., \& Kewley, L. J. 2010, ApJ, 720, 555

Passmoor, S., Cress, C., Faltenbacher, A., Johnston, R., Smith, M., Ratsimbazafy, A., \& Hoyle, A. 2013, MNRAS, 429, 2183

Patel, P., Bacon, D. J., Beswick, R. J., Muxlow, T. W. B., \& Hoyle, B. 2010, MNRAS, 401, 2572

Peacock, J. A., Schneider, P., Efstathiou, G., Ellis, J. R., Leibundgut, B., Lilly, S. J., \& Mellier, Y. 2006, ESA-ESO
Working Group on Fundamental Cosmology. Tech. rep. (astro$\mathrm{ph} / 0610906)$

Penner, K., et al. 2011, MNRAS, 410, 2749

Pérez-Torres, M. A., Alberdi, A., Romero-Cañizales, C., \& Bondi, M. 2010, A\&A, 519, L5

Petrosian, V. 2001, ApJ, 557, 560

Petrosian, V., \& East, W. E. 2008, ApJ, 682, 175

Pindor, B., Wyithe, J. S. B., Mitchell, D. A., Ord, S. M., Wayth, R., \& Greenhill, L. J. 2011, PASA, 28, 46

Prandoni, I. 2010, ISKAF2010 Science Meeting (arXiv:1008.4918)

Prandoni, I., Bernardi, G. Di Vincenzo, A., \& de Bruyn, A.G. 2012, A\&A, submitted

Prandoni, I., de Ruiter, H. R., \& Parma, P. 2007, Exploring the Cosmic Frontier: Astrophysical Instruments for the 21st Century, ESO Astrophysics Symposia, ed. A. P. Lobanov et al. (Berlin: Springer), 139

Prandoni, I., Morganti, R., \& Mignano, A. 2009, PoS, 36

Prandoni, I., Gregorini, L., Parma, P., de Ruiter, H. R., Vettolani, G., Zanichelli, A., Wieringa, M. H., Ekers, R. D. 2001, A\&A, 369, 787

Prandoni, I., Parma, P., Wieringa, M. H., de Ruiter, H. R., Gregorini, L., Mignano, A., Vettolani, G., \& Ekers, R. D. 2006, A\&A, 457, 517

Prandoni, I., de Ruiter, H. R., Ricci, R., Parma, P., Gregorini, L., \& Ekers, R. D. 2010, A\&A, 510, A42

Raccanelli, A., et al. 2012, MNRAS, 424, 801

Ransom, R. R., Kothes, R., Wolleben, M., \& Landecker, T. L. 2010, ApJ, 724, 946

Ransom, R. R., Uyanıker, B., Kothes, R., \& Landecker, T. L. 2008, ApJ, 684, 1009

Rau, U., \& Cornwell, T. J. 2010 PhD thesis, New Mexico Inst. Mining Tech.

Ravi, V., Hallinan, G., Hobbs, G., \& Champion, D. J. 2011, ApJ, 735, L2

Ravi, V., et al. 2010, MNRAS, 408, L99

Rawlings, S., Willott, C. J., Hill, G. J., Archibald, E. N., Dunlop, J. S., \& Hughes, D. H. 2004, MNRAS, 351, 676

Rayner, D. P., Norris, R. P., \& Sault, R. J. 2000, MNRAS, 319, 484

Reddy, \& Yun, 2004, ApJ, 600, 695

Reuland, M., Röttgering, H., van Breugel, W., \& De Breuck, C. 2004, MNRAS, 353, 377

Reuland, M., et al. 2003, ApJ, 592, 755

Reuland, M., et al. 2007, AJ, 133, 2607

Rezzolla, L., Barausse, E., Dorband, E. N., Pollney, D., Reis-swig, C., Seiler, J., \& Husa, S. 2008, PhRvD, 78, 044002

Richards, G. T., et al. 2005, MNRAS, 360, 839

Richter, G. A. 1975, AN, 296, 65

Rickett, B. J. 1986, ApJ, 307, 564

Rigby, E. E., Best, P. N., Brookes, M. H., Peacock, J. A., Dunlop, J. S., Röttgering, H. J. A., Wall, J. V., \& Ker, L. 2011, MNRAS, 416, 1900

Rocca-Volmerange, B., Le Borgne, D., De Breuck, C., Fioc, M., \& Moy, E. 2004, A\&A, 415, 931

Rosati, P., et al. 2002, ApJ, 566, 667

Röttgering, H., 2010a, PoS (ISKAF2010)050

Röttgering, H., et al. 2010b, http://www.astron.nl/radioobservatory/APERTIF-eoi-abstracts-and-contact-information

Roy, A. L., Norris, R. P., Kesteven, M. J., Troup, E. R., \& Reynolds, J. E. 1998, MNRAS, 301, 1019

Ryle, M., Hine, G., Shakeshaft, J., \& Caswell, J. L. 1978, Natur, 276,571 
Ryu, D., Kang, H., Cho, J., \& Das, S. 2008, Sci, 320, 909

Sabater, J., Leon, S., Verdes-Montenegro, L., Lisenfeld, U., Sulentic, J., \& Verley, S. 2008, A\&A, 486, 73

Sabater, J., Verdes-Montenegro, L., Leon, S., Best, P., \& Sulentic, J. 2012, A\&A, 545, A15

Sachs, R. K., \& Wolfe, A. M. 1967, ApJ, 147, 73

Sadler, E. M., et al. 2007, MNRAS, 381, 211

Salvato, M., et al. 2009, ApJ, 690, 1250

Salvato, M., et al. 2011, ApJ, 742, 61

Scaife, A., et al. 2010, http://www.astron.nl/radio-observatory/ APERTIF-eoi-abstracts-and-contact-information

Scalo, J., \& Elmegreen, B. G. 2004, ARA\&A, 42, 275

Schekochihin, A. A., Cowley, S. C., Kulsrud, R. M., Hammett, G. W., \& Sharma, P. 2005, ApJ, 629, 139

Schinnerer, E., et al. 2007, ApJS, 172, 46

Schmidt, S. J., Connolly, A. J., \& Hopkins, A. M. 2006, ApJ, 649, 63

Schnitzeler, D. H. F. M. 2010, MNRAS, 409, L99

Schnitzeler, D. H. F. M., Katgert, P., \& de Bruyn, A. G. 2009, A\&A, 494, 611

Schnitzeler, D. H. F. M., Katgert, P., Haverkorn, M., \& de Bruyn, A. G. 2007, A\&A, 461, 963

Scoville, N. 2007, ApJS, 172, 1

Seaquist, E. R., Krogulec, M., \& Taylor, A. R. 1993, ApJ, 410, 260

Seymour, N., et al. 2007, ApJS, 171, 353

Seymour, N., et al. 2008, MNRAS, 386, 1695

Seymour, N., et al. 2011, MNRAS, 413, 1777

Shanks, T., 2005, VST-ATLAS proposal, http://www.astro.dur.ac. uk/Cosmology/vstatlas/

Shao, L., et al. 2010, A\&A, 518, L26

Shaver, P. A., Wall, J. V., Kellermann, K. I., Jackson, C. A., \& Hawkins, M. R. S. 1996, Natur, 384, 439

Silk, J., \& Rees, M. J. 1998, A\&A, 331, L1

Simonetti, J. H., \& Cordes, J. M. 1990, ApJ, 349, 97

Slee, O. B., Wilson, W., \& Ramsay, G. 2008, PASA, 25, 94

Smirnov, O. M. 2011 A\&A, 527, A108

Smith, D. J. B., et al. 2011, MNRAS, 416, 857

Smolčić, V., et al. 2008, ApJS, 177, 14

Smolčić, V., et al. 2009, ApJ, 696, 24

Starck, J.-L., Donoho, D. L., \& Candés, E. J. 2003, A\&A, 398, 785

Starck, J. L., Murtagh, F., \& Fadili, M.-J. 2010, Sparse Image and Signal Processing: Wavelets, Curvelets, Morphological Diversity (Cambridge, UK: Cambridge University Press)

Stevens, J. A., et al. 2003, Natur, 425, 264

Stewart, I. M., Fenech, D. M., \& Muxlow, T. W. B. 2011, A\&A, 535, A81

Stil, J. M., Krause, M., Beck, R., \& Taylor, A. R. 2009, ApJ, 693, 1392

Stil, J. M., Taylor, A. R., \& Sunstrum, C. 2011, ApJ, 726, 4

Subramanian, K., Shukurov, A., \& Haugen, N. E. L., 2006, MNRAS 366,1437

Suksmono, A. 2009, ICEEI, 1, 110116

Sun, X. H., Reich, W., Waelkens, A., \& Enßlin, T. A. 2008, A\&A, 477, 573

Sutherland, W., \& Saunders, W. 1992, MNRAS, 259, 413

Taylor, A. R., \& Gregory, P. C. 1983, AJ, 88, 1784

Taylor, A. R., Stil, J. M., \& Sunstrum, C. 2009, ApJ, 702, 1230

Taylor, A. R., et al. 2003, AJ, 125, 3145

The Dark Energy Survey Collaboration 2005, arXiv:astro$\mathrm{ph} / 0510346$
Tielens, A. G. G. M., Miley, G. K., \& Willis, A. G. 1979, A\&AS, 35,153

Tingay, S. J., et al. 2012, PASA, accepted (arXiv:1206.6945)

Trigilio, C., Leto, P., Leone, F., Umana, G., \& Buemi, C. 2000, A\&A, 362, 281

Trigilio, C., Leto, P., Umana, G., Buemi, C. S., \& Leone, F. 2008, MNRAS, 384, 1437

Trigilio, C., Leto, P., Umana, G., Buemi, C. S., \& Leone, F. 2011, ApJ, 739, L10

Trott, C. M., Wayth, R. B., Macquart, J.-P., Tingay, S. J. 2012, IAUS, 285,414

Umana, G., Buemi, C. S., Trigilio, C., \& Leto, P. 2005, A\&A, 437, L1

Umana, G., Trigilio, C., \& Catalano, S. 1998, A\&A, 329, 1010

Umana, G., Trigilio, C., Tumino, M., Catalano, S., \& Rodonó, M. 1995 A\&A, 298, 143

Uyanıker, B., Kothes, R., \& Brunt, C. M. 2002, ApJ, 565, 1022

van Breugel, W., De Breuck, C., Stanford, S. A., Stern, D., Röttgering, H., \& Miley, G. ApJ, 518, L61

Van der Heyden, K., \& Jarvis, M. J. 2010, MIGHTEE proposal to MeerKAT

Van Eck, C. L., et al. 2011, ApJ, 728, 97

van Straten, W. 2006, ApJ, 642, 1004

van Weeren, R. J., Röttgering, H. J. A., Brüggen, M., \& Hoeft, M. 2010, Sci 310, 347

Vannier, M., Mary, D., Millour, F., Petrov, R. G., Bourguignon, S., \& Theys, C. 2010, Proc. SPIE 7734, 79

Venemans, et al. 2007, A\&A, 461, 823

Venturi, T. 2011, arXiv:1102.1572

Venturi, T., Giacintucci, S., Dallacasa, D., Cassano, R., Brunetti, G., Bardelli, S., \& Setti, G. 2008, A\&A, 484, 327

Verdes-Montenegro, L., Sulentic, J., Lisenfeld, U., Leon, S., Espada, D., Garcia, E., Sabater, J., \& Verley, S. 2005, A\&A, 436, 443

Vogt, C., \& Enßlin, T. A. 2005, A\&A, 434, 67

Waddington, I., Dunlop, J. S., Peacock, J. A., \& Windhorst, R. A. 2001, MNRAS, 328, 882

Waddington, I., Windhorst, R. A., Cohen, S. H., Partridge, R. B., Spinrad, H., \& Stern, D. 1999, ApJ, 526, L77

Wake, D. A., Croom, S. M., Sadler, E. M., \& Johnston, H. M. 2008, MNRAS, 391, 1674

Wakker, B. P., \& Schwarz, U. J. 1988, A\&A, 200, 312

Wall, J. 2008, arXiv:0807.3792

Wardle, J. F. C., \& Miley, G. K. 1974, A\&A, 30, 305

Wenger, S., Darabi, S., Sen, P., Glassmeier, K. H., \& Magnor, M. 2010, in Proc. IEEE Int. Conf. on Image Process., IEEE Signal Process. Soc., 1381

White, R. L., Helfand, D. J., Becker, R. H., Glikman, E., \& de Vries, W. 2007, ApJ, 654, 99

Whiting, M. 2012, MNRAS, 421, 3242

Wiaux, Y., Jacques, L., Puy, G., Scaife, A. M. M., \& Vandergheynst, P. 2009a, MNRAS, 395, 1733

Wiaux, Y., Puy, G., Boursier, Y., \& Vandergheynst, P. 2009b, MNRAS, 400, 1029

Widrow, L. M. 2002, RvMP, 74, 775

Willott, C. J., Rawlings, S., Jarvis, M. J., \& Blundell, K. M. 2003, MNRAS, 339, 173

Wilman, R. J., Jarvis, M. J., Mauch, T., Rawlings, S., \& Hickey, S. 2010, MNRAS, 405, 447

Wilman, R. J., et al. 2008, MNRAS, 388, 1335

Wilson, A. S., \& Colbert, E. J. M. 1995, ApJ, 438, 62 
Wilson, G., et al. 2008, ASPC, 381, 210

Wing, J. D., \& Blanton, E. L. 2011, AJ, 141, 88

Wright, E. L., et al. 2010, AJ, 140, 1868

Yun, M. S., Reddy, N. A., \& Condon, J. J. 2001, ApJ, 554, 803

Zhao, G.-B., Giannantonio, T., Pogosian, L., Silvestri, A., Bacon,
D. J., Koyama, K., Nichol, R. C., \& Song, Y.-S. 2010, PhRvD, 81,103510

Zinn, P.-C., Middelberg, E., \& Ibar, E. 2011, A\&A, 531, A14

Zinn, P.-C., Middelberg, E., Norris, R. P., Hales, C. A., Mao, M. Y., \& Randall, E. K. 2012a, A\&A, 544, A38 